UNIVERSITY OF ROCHESTER

LABORATORY FOR LASER ENERGETICS
Volume 77 October-December 1998 DOE/SF/19460-284

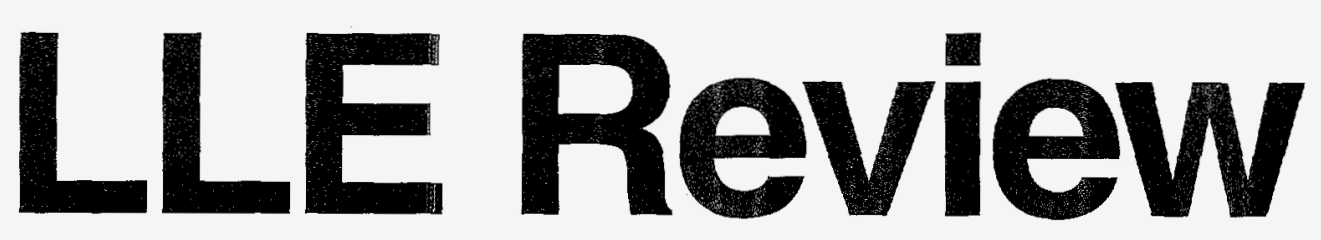

Quarterly Report
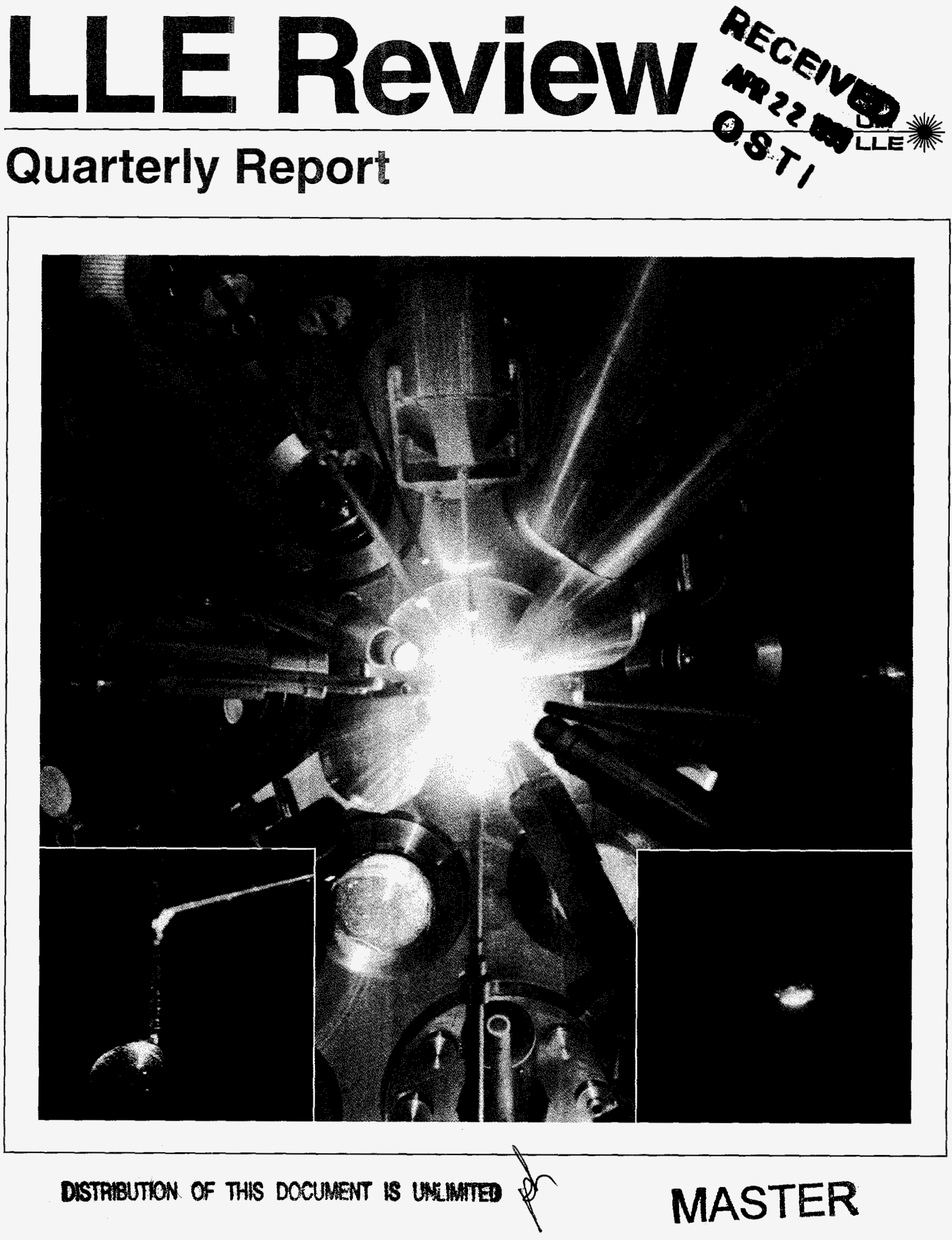


\section{About the Cover:}

The central photograph illustrates the OMEGA target chamber during the experimental campaign to study laser-plasma interactions in long-scale-length plasmas with parameters relevant to the peak of the direct-drive National Ignition Facility laser pulses. (Key elements of the target chamber are defined in the photograph below.) The $\mathrm{CH}$ target is suspended from the positioner seen at the top of the chamber; magnified views of the solid $1.5-\mathrm{mm}$-diam partial sphere before and during the 12-kJ laser pulse are shown in the left and right insets, respec-

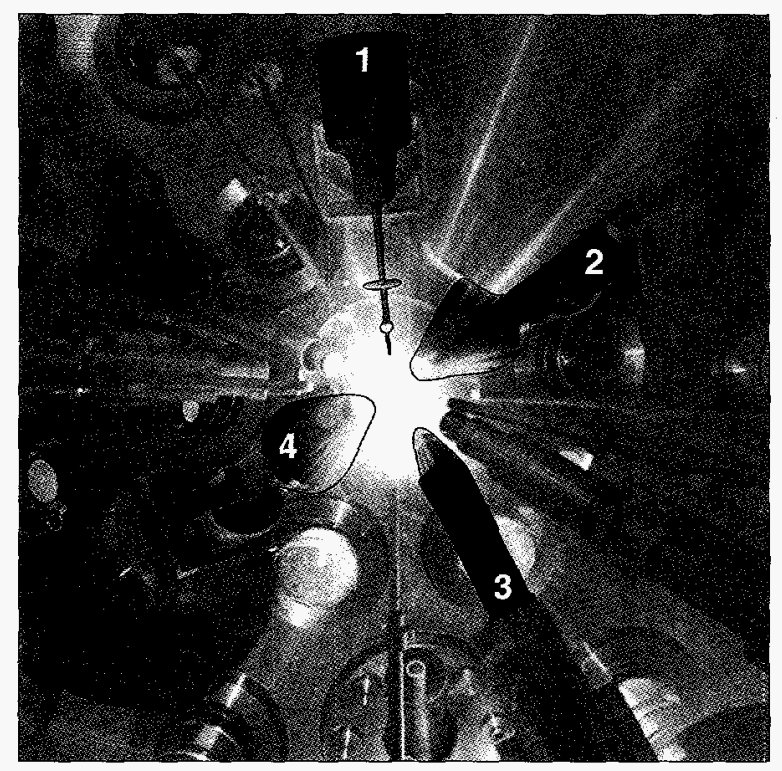

This report was prepared as an account of work conducted by the Laboratory for Laser Energetics and sponsored by New York State Energy Research and Development Authority, the University of Rochester, the U.S. Department of Energy, and other agencies. Neither the above named sponsors, nor any of their employees, makes any warranty, expressed or implied, or assumes any legal liability or responsibility for the accuracy, completeness, or usefulness of any information, apparatus, product, or process disclosed, or represents that its use would not infringe privately owned rights. Reference herein to any specific commercial product, process, or service by trade name, mark, manufacturer, or otherwise, does not necessarily constitute or imply its endorsement, recommendation, or fa-

Printed in the United States of America

Available from

National Technical Information Services

U.S. Department of Commerce

5285 Port Royal Road

Springfield, VA 22161

Price codes: Printed Copy A04

Microfiche A01 tively. The interaction beam is delivered to the plasma from the bottom of the target chamber, and stimulated Raman scattering and stimulated Brillouin scattering signals are collected with the f/6 OMEGA focusing lens of the interaction beam and relayed to time-resolved visible spectrometers (not shown). $K$-shell spectra of $\mathrm{Ti}$ and $\mathrm{Ca}$ embedded microdots, recorded with the TIMbased $x$-ray diagnostics, are used to characterize the plasma electron temperature.

The photograph on the left defines the layout of the central cover photograph of the OMEGA target chamber by highlighting the key elements. The target positioner and the streaked $x$-ray crystal spectrograph are labeled 1 and 3, respectively, and the two time-integrated crystal spectrographs are labeled 2 and 4 .

voring by the United States Government or any agency thereof or any other sponsor. Results reported in the LLE Review should not be taken as necessarily final results as they represent active research. The views and opinions of authors expressed herein do not necessarily state or reflect those of any of the above sponsoring entities.

The work described in this volume includes current research at the Laboratory for Laser Energetics, which is supported by New York State Energy Research and Development Authority, the University of Rochester, the U.S. Department of Energy Office of Inertial Confinement Fusion under Cooperative Agreement No. DE-FC03-92SF19460, and other agencies.

For questions or comments, contact Sean P. Regan, Editor, Laboratory for Laser Energetics, 250 East River Road, Rochester, NY 14623-1299, (716) 275-7077; e-mail: sreg@lle.rochester.edu.

Worldwide-Web Home Page: http://www.lle.rochester.edu/ 


\section{DISCLAMMER}

This report was prepared as an account of work sponsored by an agency of the United States Government. Neither the United States Government aor any agency thereof, nor any of their employees, makes any warranty, express or implied, or assumes any legal liability or responsibility for the accuracy, completeness, or usefulness of any information, apparatus, product, or process disclosed, or represents that its use would not infringe privately owned rights. Reference berein to any specific commercial produch, process, or service by trade name, trademark, inanufacturer, or otherwise does not necessarily constitute or imply its endorsement, recommendation, or favoring by the United States Governmeat or any ageacy thereof. The views and opinions of authors expressed herein do not necessarily state or reflect those of the United States Government or any agency thereof. 


\section{DISCLAIMER}

Portions of this document may be illegible in electronic image products. Images are produced from the best available original document. 


\section{Quarterly Report}

\section{Contents}

In Brief

Laser-Plasma Interactions in Long-Scale-Length Plasmas

Under Direct-Drive National Ignition Facility Conditions.

Irradiation Uniformity for High-Compression Laser-

Fusion Experiments

A Novel Charged-Particle Diagnostic for $\rho R$ in Compressed ICF Targets

Arresting UV-Laser Damage in Fused Silica 26

Theory of the Ablative Richtmyer-Meshkov Instability

Reverse Intersystem Crossing from a Triplet State of Rose Bengal Populated by Sequential 532-nm plus 1064-nm Laser Excitation

Picosecond Response of Optically Driven Y-Ba-Cu-O

Microbridge and Josephson-Junction Integrated Structures

Publications and Conference Presentations 



\section{In Brief}

This volume of the LLE Review, covering the period October-December 1998, includes two articles addressing issues applicable to direct-drive ICF on the National Ignition Facility (NIF): laser-plasma interactions and laser-irradiation uniformity. In the first article S. P. Regan, R. S. Craxton, D. D. Meyerhofer, W. Seka, R. W. Short, A. Simon, and B. Yaakobi present experimental results that indicate the parametric instabilities stimulated Raman scattering and stimulated Brillouin scattering are not likely to have a significant impact on target performance at the peak of the NIF direct-drive laser pulse. In addition, the production and characterization of long-scale-length plasmas, with parameters relevant to the peak of the direct-drive NIF laser pulses, on the OMEGA laser system are described. In the second article S. Skupsky and R. S. Craxton investigate laser-irradiation uniformity on OMEGA and the NIF and outline improvements in irradiation uniformity planned for the OMEGAlaser during 1999 that will reduce the rms nonuniformity to less than $1 \%$ when the intensity is averaged over $300 \mathrm{ps}$. Higher uniformity will be achievable on the NIF due to the larger number of beams (192 versus 60 ).

Additional highlights of the research presented in this issue are

- P. B. Radha and S. Skupsky present a novel charged-particle diagnostic that performs simultaneous $\rho R$ measurements of the fuel, shell, and ablator regions of a compressed ICF target, consisting of an inner DT fuel region, a plastic (CH) shell, and an ablator (CD), by measuring the knock-on deuteron spectrum. This diagnostic technique relies on the new charged-particle spectrometer on OMEGA to obtain the particle spectrum.

- F. Dahmani, S. Burns, J. Lambropoulos, S. Papernov, and A. Schmid report results from stressinhibited laser-driven crack propagation and stress-delayed damage-initiation experiments in fused silica at $351 \mathrm{~nm}$. The damage initiation threshold was observed to increase by $70 \%$ when a modest amount of mechanical stress was applied to the fused-silica optic. Research is underway presently to determine the ramifications of these findings for large-aperture systems, such as OMEGA.

- V. Goncharov presents an analytic theory of the ablative Richtmyer-Meshkov instability, which shows that the main stabilizing mechanism of the ablation-front perturbations is the dynamic overpressure of the blowoff plasma with respect to the target material. The perturbation evolution during the shock transit time is studied to determine the initial conditions for the Rayleigh-Taylor phase of the instability and to analyze the level of laser imprint on ICF direct-drive targets.

- J. M. Larkin, W. R. Donaldson, T. H. Foster, and R. S. Knox examine the triplet state of rose bengal, a dye used in photodynamic therapy, that is produced by $1064-\mathrm{nm}$ excitation of $\mathrm{T}_{1}$. The triplet-triplet absorption cross section between $825 \mathrm{~nm}$ and $1100 \mathrm{~nm}$ was measured, and two-step laser-induced fluorescence was used to characterize the thermalization rate, lifetime, and quantum yield of reverse intersystem crossing of the triplet state. 
- R. Adam, M. Currie, R. Sobolewski, O. Harnack, and M. Darula report measurements of the picosecond photoresponse of a current-biased YBCO microbridge coupled to a bicrystal YBCO Josephson junction. Single-pico-second switching of a high-temperature-superconductor Josephson junction was observed, and the junction turn-on delay time was measured. These findings provide confirmation of the potential of YBCO for ultrafast optical and electrical transient detection and processing.

Sean P. Regan

Editor 


\section{Laser-Plasma Interactions in Long-Scale-Length Plasmas Under Direct-Drive National Ignition Facility Conditions}

The National Ignition Facility (NIF) (currently under construction), with a nominal laser energy of $1.8 \mathrm{MJ}$, is expected to achieve ignition in both direct- 1,2 and indirect-drive ${ }^{3}$ configurations. The mission of the University of Rochester's Laboratory for Laser Energetics is to study the direct-drive approach to inertial confinement fusion (ICF), where the capsule is directly irradiated by a large number of symmetrically arranged laser beams. To validate the performance of high-gain, direct-drive target designs planned for the NIF, ${ }^{2}$ an understanding of the laser-plasma interactions in the coronal plasmas of these targets is essential. These interactions include stimulated Raman scattering (SRS), stimulated Brillouin scattering (SBS), the two-plasmon decay instability (TPD), and filamentation. ${ }^{4}$ Their significance for direct-drive capsule performance arises either from detrimental suprathermal-electron generation due to plasma wave-breaking or other nonlinear processes (SRS, TPD), or through a reduction in drive power or drive uniformity (SBS, filamentation).

When the incident laser beam intensity exceeds the threshold levels for the various parametric instabilities, power is transferred from the incident laser light to lower-frequency electromagnetic, electron-plasma, or ion-acoustic waves if the energy $\left(\omega_{0}=\omega_{1}+\omega_{2}\right)$ and momentum conservation $\left(\underline{k}_{0}=\underline{k}_{1}\right.$ $+\underline{\mathrm{k}}_{2}$ ) relationships are satisfied (phase matching). Here, $\omega_{0}$ and $\underline{\mathrm{k}}_{0}$ represent the pump-wave (laser) frequency and wave vector. The SRS decay products are an electron-plasma wave and a scattered electromagnetic wave, which are denoted by the subscripts 1 and 2 . The TPD results when the incident laser light decays into two electron-plasma waves. Phase matching occurs for SRS and the TPD at electron densities $n_{e}$ less than or equal to the quarter-critical density $n_{c} / 4$. When the phase-matching conditions are satisfied for SBS, the incident laser light decays into an ion-acoustic wave and a scattered electromagnetic wave.

A 1.5-MJ, $\alpha=3$ ( $\alpha$ is defined as the ratio of the cold fuel pressure to the Fermi-degenerate pressure) laser pulse is planned for an all-DT direct-drive target design on the NIF. ${ }^{2}$ This pulse has a peak on-target intensity $I_{\text {total }}$ of $2 \times 10^{15} \mathrm{~W} / \mathrm{cm}^{2}$ (summed over all beams) and a foot intensity of $4 \times 10^{13} \mathrm{~W} / \mathrm{cm}^{2}$. The cluster intensities $I_{\text {cluster }}$ (summed over four individual beams) are approximately a factor of 10 lower than $I_{\text {total }}$. The coronal plasma predicted for this design has an electron temperature $T_{e}$ of $\sim 4 \mathrm{keV}$, a density scale length $L_{n}\left(=n_{e} / \nabla n_{e}\right)$ of $\sim 0.75 \mathrm{~mm}$, and a velocity-gradient scale length $L_{v}\left[\left(=c_{s} / \nabla v\right)\right.$, where $c_{s}$ is the ion-acoustic velocity and $v$ is the velocity] of $\sim 0.5 \mathrm{~mm}$ at the peak of the laser pulse and at densities of $0.1 n_{c}$ to $0.2 n_{c}$. While parametric instabilities have been studied extensively under conditions relevant to indirect-drive $\mathrm{ICF}^{5,6}$ the results presented here represent their first study in NIF-scale directdrive plasmas.

This article reports on experiments under plasma conditions representative of the peak of the NIF direct-drive laser pulse, since they are the most challenging to create. The experiments were performed on the 30-kJ, 351-nm, 60-beam OMEGA laser system, ${ }^{7}$ with distributed phase plates (DPP's) ${ }^{8}$ and $f / 6$ focusing lenses on all beams. The targets, all made of $\mathrm{CH}$, included exploding foils and solids. The exploding foils produced large, millimeter-scale plasmas with $T_{e} \sim 4 \mathrm{keV}$ and a peak on-axis $n_{e}$ between $0.1 n_{c}$ and $0.2 n_{c}$. These temperatures and densities were diagnosed using time-resolved $\mathrm{x}$-ray and visible (SRS backscattering) spectroscopy, respectively, and the observations were found to be consistent with the predictions of the two-dimensional Eulerian hydrodynamics code $S A G E .{ }^{9}$ The solid-target plasmas were predicted to reach similar temperatures and to have similar density profiles below $\sim 0.1 n_{c}$; however, while they lacked the large plateau region characteristic of exploding foils, they included a critical-density surface and were in this sense more representative of NIF conditions.

When both foil and solid-target plasmas were irradiated with an interaction beam at $1.5 \times 10^{15} \mathrm{~W} / \mathrm{cm}^{2}$, the directbackscattered SBS signal was found to be completely absent. Some direct-backscattered SRS was observed in the solidtarget plasmas at a very low level, with a conservative upper limit of $\sim 5 \%$. 
In the following sections simulations of the NIF direct-drive plasmas carried out with the one-dimensional hydrodynamics code $L I L A C^{10}$ are presented; the production of long-scalelength plasmas on OMEGA is discussed together with $S A G E$ simulations; the characterization of the plasma electron temperature and density and comparisons with $S A G E$ predictions are analyzed; and the SRS and SBS measurements are presented. The main conclusion of this research is that SRS and SBS are not likely to have a significant impact on target performance at the peak of the NIF direct-drive laser pulse.

\section{NIF Direct-Drive Plasmas}

LILAC calculations of the $1.5-\mathrm{MJ}, \alpha=3$ direct-drive target are shown in Fig. 77.1. This figure gives the predicted trajectories of the imploding target, the critical-density surface, and the $n_{c} / 4$ and $n_{c} / 10$ surfaces. It is seen that the density scale length and electron temperature increase with time as the laser pulse makes the transition from the foot to the peak intensity. The dashed line shows the laser power history.

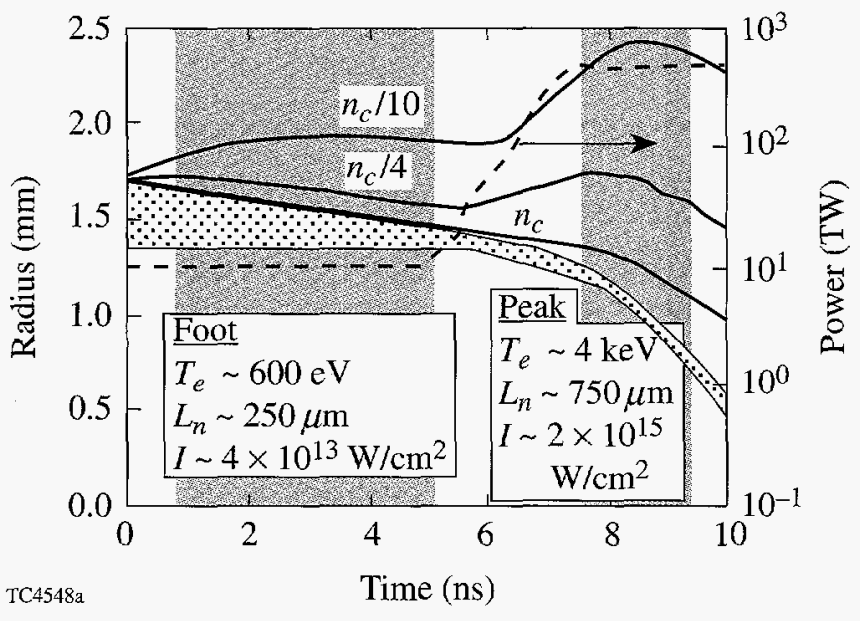

\section{Figure 77.1}

Radius versus time calculated by $L I L A C$ for a high-gain, direct-drive target planned for the NIF with $1.5 \mathrm{MJ}$ and $\alpha=3$. The dotted area represents the solid density region, and the solid lines give the trajectories of the critical, quartercritical, and tenth-critical surfaces. The dashed line indicates the laser power as a function of time. The gray areas represent the "foot" and "peak" portions of the pulse, for which the calculated values of electron temperature $T_{e}$, density scale length $L_{n}\left(=n_{e} / \nabla n_{e}\right)$, and on-target intensity $I$ (summed over all beams) are indicated.

A summary of parameters for the foot and peak of this pulse, including the predicted intensity thresholds $I_{\text {threshold }}$ for the SBS, SRS, and TPD instabilities, is given in Table 77.I. Approximations for these thresholds were taken from Kruer. ${ }^{4}$ These thresholds may be compared with the cluster intensity $I_{\text {cluster, }}$, i.e., the intensity summed over the four beams in a NIF cluster, and the total overlapped intensity $I_{\text {total }}$. In the case of SRS it is anticipated that the phase-matching conditions will be satisfied only for the light within the f/8 NIF cluster, so that the appropriate comparison is with $I_{\text {cluster }}$. In the case of SBS, more than one cluster may drive the instability, but the relevant threshold intensity is likely to be significantly less than $I_{\text {total }}$. As shown in Table 77.I, $I_{\text {cluster }}$ is less than $I_{\text {threshold }}$ for all the instabilities during the foot of the laser pulse. During the peak of the pulse $I_{\text {cluster }}$ is about an order of magnitude lower than $I_{\text {threshold }}$ for SRS and SBS. These comparisons support the expectation that significant SRS and SBS will not occur in direct-drive NIF plasmas. However, the intensities quoted here represent averages over many speckles produced by the phase plates; inside the speckles the peak intensities may be several times higher. It should also be noted that $I_{\text {cluster }}$ is 2.5 times greater than $I_{\text {threshold }}$ for the TPD instability during the peak of the pulse; thus, the TPD instability (not included in the present work because the geometrical configuration is not optimum for its study) should be investigated.

\section{Production of Long-Scale-Length Plasmas on OMEGA}

The long-scale-length plasmas created on OMEGA made use of a design similar to that used on the former 24-beam OMEGA laser system. ${ }^{11}$ The 60 OMEGA beams were divided into various groups that irradiated the target from different angles and at different times (see Figs. 77.2 and 77.3). Both exploding foils and solid targets were irradiated first by primary beams $(P)$, whose purpose is to form the plasma, and then by secondary beams $(S)$, which heat the plasma and can be used to control its temperature. The $\mathrm{CH}$ foil targets were $1.2 \mathrm{~mm}$ in diameter, a little larger than the laser spot diameter, and 18 to $20 \mu \mathrm{m}$ thick. The solid targets included 1.5 -mm-diam planar slabs and partial spheres with thicknesses greater than $80 \mu \mathrm{m}$, predicted to result in similar plasma conditions. In both cases the plasmas were irradiated by an interaction beam of variable timing, incident along the initial target normal.

The timing sequence of the various groups of beams was similar for foils and solid targets. It is shown in Fig. 77.3, which gives the time history of the incident, absorbed, and transmitted laser powers. All beams were 1-ns square pulses with various delays. The primary beams were split into two groups: $P_{1}$, from 0 to $1 \mathrm{~ns}$, with angle of incidence $\sim 20^{\circ}$, and $P_{2}$, from 1 to $2 \mathrm{~ns}$ and incident at $\sim 40^{\circ}$. For the foil targets, these beams served to explode the foils. By the end of the second group $\left(P_{2}\right)$ the foils became underdense on axis because the phase-plate focal spot distribution is Gaussian-like with its maximum in the center. This resulted in some transmitted laser power starting at $1.5 \mathrm{~ns}$, as seen in Fig. 77.3(a). The secondary beams 
Table 77.I: Parameters for the foot and peak portions of the 1.5-MJ, $\alpha=3$, direct-drive target planned for NIF, including coronal plasma conditions and intensity threshold approximations for parametric instabilities.

\begin{tabular}{|c|c|c|}
\hline \hline & Foot & Peak \\
\hline$T_{e}$ & $0.6 \mathrm{keV}$ & $4 \mathrm{keV}$ \\
\hline$L_{n}$ & $0.25 \mathrm{~mm}$ & $0.75 \mathrm{~mm}$ \\
\hline$L_{v}$ & $0.5 \mathrm{~mm}$ & $0.5-1.0 \mathrm{~mm}$ \\
\hline$I_{\text {total }}$ & $4 \times 10^{13} \mathrm{~W} / \mathrm{cm}^{2}$ & $2 \times 10^{15} \mathrm{~W} / \mathrm{cm}^{2}$ \\
\hline$I_{\text {cluster }}$ & $4 \times 10^{12} \mathrm{~W} / \mathrm{cm}^{2}$ & $2 \times 10^{14} \mathrm{~W} / \mathrm{cm}^{2}$ \\
\hline$I_{\text {threshold }}($ SBS $)$ & $4.6 \times 10^{14} \mathrm{~W} / \mathrm{cm}^{2}$ at $n_{c} / 10$ & $2 \times 10^{15} \mathrm{~W} / \mathrm{cm}^{2}$ at $n_{c} / 10$ \\
\hline$I_{\text {threshold }}($ SRS) & $5.0 \times 10^{15} \mathrm{~W} / \mathrm{cm}^{2}$ & $1.7 \times 10^{15} \mathrm{~W} / \mathrm{cm}^{2}$ \\
\hline$I_{\text {threshold }}(\mathrm{TPD})$ & $3.4 \times 10^{13} \mathrm{~W} / \mathrm{cm}^{2}$ & $8 \times 10^{13} \mathrm{~W} / \mathrm{cm}^{2}$ \\
\hline \hline
\end{tabular}

(a)

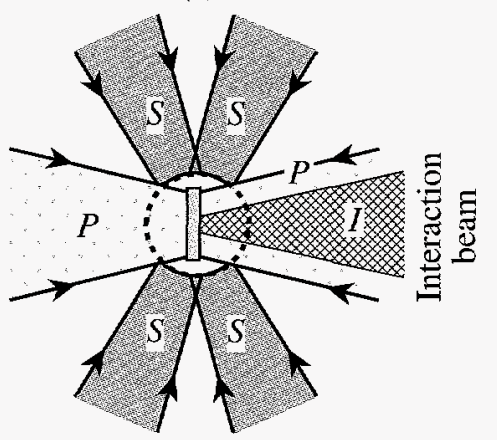

E8705a

Exploding foil (b)

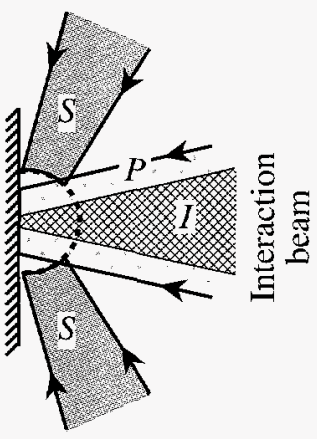

Solid target

Figure 77.2

Geometry for the formation of long-scale-length plasmas on the OMEGA laser system. (a) A CH foil is irradiated and exploded with primary $(P)$ and (delayed) secondary $(S$ ) laser beams. (b) A solid $\mathrm{CH}$ target is irradiated with primary and secondary laser beams from one side. In both cases the plasma (shown schematically by the dashed curves) is irradiated by an interaction beam $(I)$ with variable timing.

arrived from 2 to $3 \mathrm{~ns}$. Their absorption fraction is typically high, and they are effective at heating a plasma whose volume is $\sim 1 \mathrm{~mm}^{3}$. For the exploding foils, the transmitted power increases later in time as the plasma expands. The interaction beam typically provides little heating to the plasma and little perturbation to the hydrodynamics, the exception being the solid target after the end of the secondary beams when the interaction beam, if still present, is strongly absorbed and provides some localized heating.

The on-target laser energy was typically $500 \mathrm{~J}$ per beam. The exploding-foil plasmas were irradiated with a total of $19 \mathrm{~kJ}$ of laser energy from 38 beams. Depending on the number of secondary beams used, the solid targets were irradiated with either 19 or 29 beams from one side to deliver 8 to $12 \mathrm{~kJ}$, producing plasmas with predicted electron temperatures of 3 and $4 \mathrm{keV}$, respectively.

The DPP's used on all beams except the interaction beam produced a focal-spot intensity distribution whose envelope was approximately Gaussian in shape with a full-width-athalf-maximum diameter of $480 \mu \mathrm{m}$ and a diameter of $950 \mu \mathrm{m}$ enclosing $95 \%$ of the energy. The interaction beam used a different DPP, designed to produce a similar focal-spot shape but scaled down a factor of 2.8 in diameter. The peak intensity in space and time for a nominal 500 -J beam was $1.6 \times 10^{15}$ $\mathrm{W} / \mathrm{cm}^{2}$ for the interaction beam and $2.1 \times 10^{14} \mathrm{~W} / \mathrm{cm}^{2}$ for the other beams. On most shots 2-D SSD ${ }^{12}$ was used (on all beams), with a bandwidth of $0.25 \mathrm{THz}$ in the UV.

Contour plots of the predicted $T_{e}$ and $n_{e}$ at $2.6 \mathrm{~ns}$ are shown in Fig. 77.4 for the two types of plasma. Below eighth-critical density, the two plasmas have very similar density, temperature, and velocity profiles. These are similar to those anticipated for the NIF direct-drive target. For the exploding-foil plasma the density profile on axis has a full width at halfmaximum of $1 \mathrm{~mm}$. For the solid target, the scale length $L_{n} \sim 0.5 \mathrm{~mm}$. 
One interesting feature seen in the density contour plot of Fig. 77.4(a) for the exploding foil is a high-density off-axis region, topologically a ring structure. This effect was also found in earlier experiments on the former 24-beam OMEGA
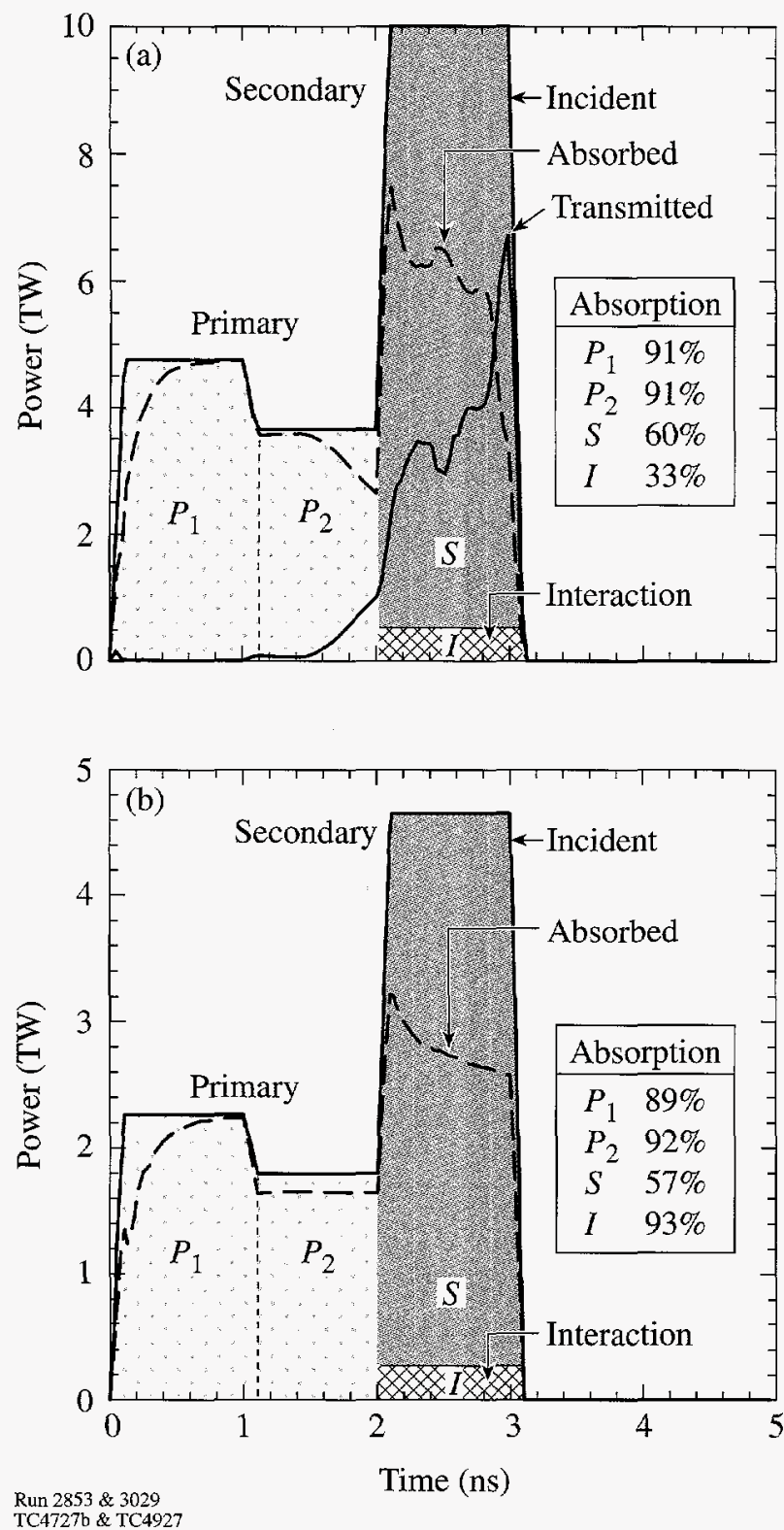

Figure 77.3

Timing sequence and total power delivered to the target for 1-ns flat-top laser pulses used to irradiate (a) exploding foils and (b) solid targets. The interaction beam is fired to probe the NIF-relevant plasma conditions, which usually occur between 2 and $3 \mathrm{~ns}$, and can be timed to start anywhere from 2 to $3 \mathrm{~ns}$. The figure also shows the calculated absorbed and transmitted powers as functions of time, summed over all beams, and the time-integrated absorption fractions of the primary $\left(P_{1}, P_{2}\right)$, secondary $(S)$, and interaction $(I)$ beams. system. ${ }^{11}$ lt occurs because the centrally peaked primary beams cause a more rapid explosion of the on-axis portion of the foil. The edge of the foil is only weakly heated by the primary beams. The off-axis ring expands because of heating by the obliquely incident secondary beams, resulting in a flow of mass toward the axis. This compensates for what would otherwise be a rapid fall of density with respect to time in the center of the plasma.

The predicted temporal evolution of the temperature and density in the center of an exploding-foil plasma is plotted in Fig. 77.5. The temperature rises rapidly around $1 \mathrm{~ns}$ when the thermal front penetrates to the center, and it rises again at $2 \mathrm{~ns}$ when the secondary beams turn on. It falls rapidly at $3 \mathrm{~ns}$ when the secondary beams switch off. The density, which has fallen to $\sim n_{c} / 5$ at the start of the secondary beams, stays between $n_{c} / 5$ and $n_{c} / 10$ for the next 2 ns as a result of the flow of mass from the off-axis ring seen in Fig. 77.4(a). NIF-relevant conditions are thus maintained throughout the time period of the secondary beams ( 2 to $3 \mathrm{~ns}$ ).

\section{Plasma Characterization}

The electron temperature of the exploding-foil plasmas was diagnosed using time-resolved $\mathrm{x}$-ray spectroscopy. High- $Z$ microdots, composed of $\mathrm{Ti}$ and $\mathrm{CaF}(1000 \AA$ A thick and $200 \mu \mathrm{m}$ in diameter), were embedded in the center of the foil targets. They served as tracer elements to diagnose the electron temperature predicted in Fig. 77.5(a), using the sensitivity of the (a) Exploding foil
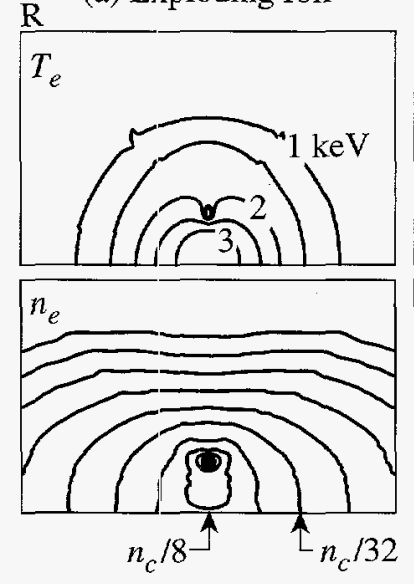

Runs 3031,3029 TC4895 (b) Solid target
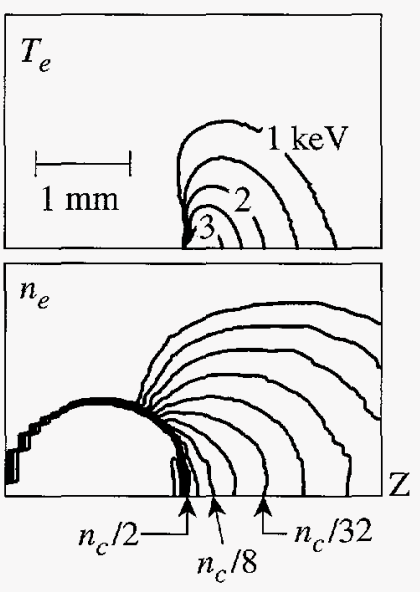

Figure 77.4

Contour plots of $T_{e}$ and $n_{e}$ at $2.6 \mathrm{~ns}$ simulated with $S A G E$ for (a) an $18-\mu \mathrm{m}$ thick exploding foil and (b) a solid target (a 2-mm-diam $\mathrm{CH}$ sphere). 
$K$-shell emission lines of Ti and $\mathrm{Ca}$ to variations in the electron temperature for temperatures up to the predicted maximum of $\sim 4 \mathrm{keV}$. Exploding-foil plasmas were used for the temperature measurement because the tracer elements diagnosed the center of the plasma. In a solid target, a tracer element embedded at some depth in the target moves a significant distance through the corona during the interaction, encountering a wide range of electron temperatures and densities, and interpretation is more complex. Targets without microdots were investigated to verify that the microdot did not affect the parametric instabilities or plasma hydrodynamics in a significant manner.
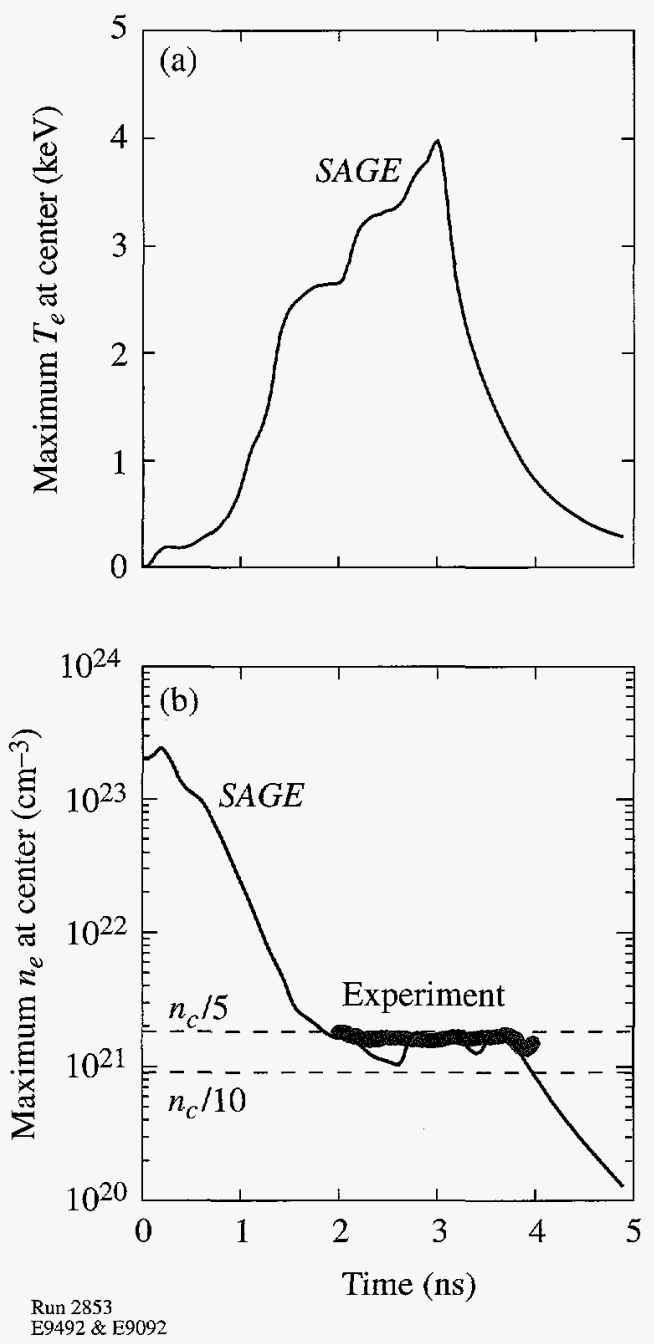

Figure 77.5

$S A G E$ predictions for the time dependence of (a) $T_{e}$ and (b) $n_{e}$ at the center of an exploding-foil plasma. This temperature was diagnosed using a tracer layer containing $\mathrm{Ti}$ and $\mathrm{Ca}$ placed in the center of the target. The electron density inferred from the streaked Raman spectrum for a number of shots with different interaction beam timings is also plotted.
Streaked and high-resolution, time-integrated $\mathrm{x}$-ray spectra of $\mathrm{Ti}$ and $\mathrm{Ca}$ tracer elements in exploding-foil plasmas were recorded with flat crystal spectrographs. The time-resolved measurement shown in Fig. 77.6(a) was photometrically calibrated for spectral sensitivity with the time-integrated measurement shown in Fig. 77.6(b). The spectral range was selected to cover the $K$-shell emission of Ti and $\mathrm{Ca}$. Both instruments had similar views of the plasma. The time-integrated instrument, which was calibrated using measured crystal reflectivities and published film sensitivities, utilized an ADP (ammonium dihydrogen phosphate) or a PET (pentaerythritol) crystal to
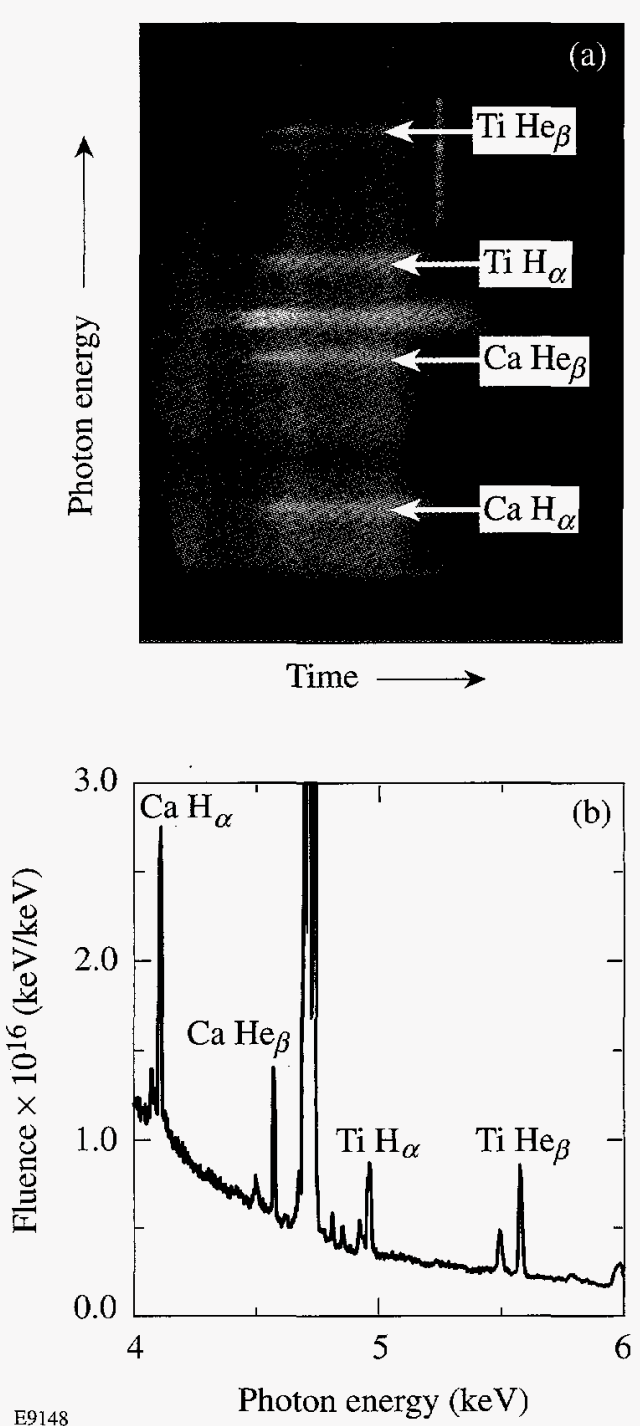

Figure 77.6

(a) Streaked $x$-ray spectra and (b) high-resolution, time-integrated $x$-ray spectra of $\mathrm{Ti}$ and $\mathrm{Ca}$ in a typical exploding-foil plasma. 
disperse the spectrum onto Kodak DEF (direct exposure) film. The $\mathrm{x}$-ray streak camera ${ }^{13}$ utilized a RbAP (rubidium acid phthalate) crystal to disperse the spectrum onto a fluffy $\mathrm{KBr}$ photocathode with a 30-ps temporal resolution. The spectra were recorded on Kodak T-max $3200 \mathrm{film}$. The sweep speed of the streak camera was measured using a temporally modulated fiducial pulse. The film from both instruments was digitized using a PDS (Perkin-Elmer Photometric Data Systems) microdensitometer and corrected for film sensitivity.

The electron temperature was diagnosed using the measured line ratios of hydrogen- and helium-like charge states of $\mathrm{Ca}$ and $\mathrm{Ti}$. In the exploding-foil plasmas the ionization time of the relevant $\mathrm{Ca}$ and $\mathrm{Ti}$ ions is longer than the interaction time $(\sim 1 \mathrm{~ns})$; therefore, a non-steady-state calculation of the evolution of the line intensities was required. Using the timedependent temperature and density predicted by $S A G E$ for the target center (see Fig. 77.5), the evolution of the diagnostic lines $\left(\mathrm{H}_{\alpha}\right.$ and $\mathrm{He}_{\beta}$ of $\mathrm{Ti}$ and $\left.\mathrm{Ca}\right)$ was calculated with the timedependent $F L Y$ atomic physics code ${ }^{14}$ (based on Ref. 15), which solves the ionic rate equations including ionization, excitation, and recombination (radiative, collisional, and dielectronic). The SAGE/FLY predictions of the $\mathrm{Ti}$ and $\mathrm{Ca}$ $\mathrm{H}_{\alpha}: \mathrm{He}_{\beta}$ emission line ratios (shaded region) are plotted in Fig. 77.7 with the measured line ratios (circle symbols). The shaded region is bounded by the FLY predictions for the SAGEpredicted $T_{e}$ and 1.2 times the $S A G E$-predicted $T_{e}$. The Ti measurement extends from just below the lower curve at $2 \mathrm{~ns}$ to just below the upper curve at $3 \mathrm{~ns}$, and the Ca measurement is closer to the upper curve at all times. Based on this agreement with $S A G E / F L Y$ predictions for both line ratios, a peak $T_{e}$ of $\sim 4 \mathrm{keV}$ is inferred. The opacity of these lines due to selfabsorption was established experimentally to be insignificant: when the thickness of the microdot was doubled, the measured intensity doubled. The isoelectronic method ${ }^{16}$ for measuring the electron temperature was not available due to uncertainty in the relative amounts of $\mathrm{Ti}$ and $\mathrm{Ca}$.

The peak plasma electron density in the exploding-foil plasmas was diagnosed using the near-backscattered SRS spectrum. ${ }^{4}$ In the center of an exploding foil, SRS is an absolute instability at the peak of the parabolic profile and consequently has an intensity threshold of $7 \times 10^{13} \mathrm{~W} / \mathrm{cm}^{2}$. A narrow spectral emission was observed with a time-resolved spectrograph [see Fig. 77.8(a)]. The observed SRS wavelength determined from the $50 \%$ intensity point on the long-wavelength side of the narrow peak is compared with the wavelength predicted from a $S A G E$ simulation in Fig. 77.8(b), using the following relationship between the density and the scattered wavelength:

$$
\frac{n}{n_{c}}=\left(1-\frac{\lambda_{0}}{\lambda_{s}}\right)^{2}+3 \frac{v_{T}^{2}}{c^{2}} \frac{\left(1-4 \frac{\lambda_{0}}{\lambda_{s}}+\frac{\lambda_{0}^{2}}{\lambda_{s}^{2}}\right)}{1-12 \frac{v_{T}^{2}}{c^{2}}}
$$

$$
-3 \frac{v_{T}^{2}}{c^{2}} \frac{\sqrt{\left(1-4 \frac{\lambda_{0}}{\lambda_{s}}+\frac{\lambda_{0}^{2}}{\lambda_{s}^{2}}\right)^{2}-\left(1-12 \frac{v_{T}^{2}}{c^{2}}\right)\left(1-\frac{\lambda_{0}^{2}}{\lambda_{s}^{2}}\right)}}{1-12 \frac{v_{T}^{2}}{c^{2}}}
$$
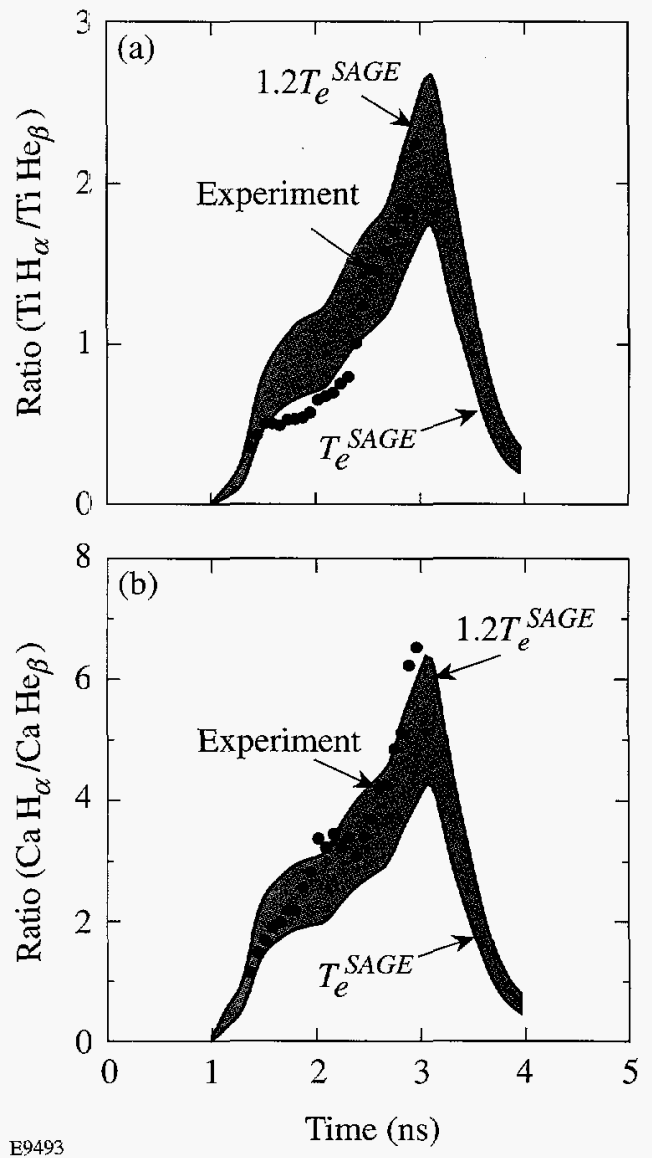

Figure 77.7

$S A G E / F L Y$ predictions of the $\mathrm{Ti}$ and $\mathrm{Ca}_{\alpha}: \mathrm{He}_{\beta}$ emission Iine ratios (shaded region) together with the measured line ratios (circle symbols). The shaded region is bounded on the lower side by the FLY prediction using the $S A G E$ predicted $T_{e}$ and on the upper side by the $F L Y$ prediction for 1.2 times the $S A G E T_{e}$. Agreement with $S A G E / F L Y$ predictions is found for the measured $\mathrm{Ti}$ and $\mathrm{Ca}$ line ratios, indicating a peak $T_{e}$ of $\sim 4 \mathrm{keV}$. 
where $v_{T}$ is the electron thermal velocity, $c$ is the speed of light, $\lambda_{0}$ is the wavelength of the incident laser light, and $\lambda_{s}$ is the scattered wavelength. This equation was obtained from the dispersion relations and phase-matching conditions for all the waves involved in the SRS.

The experimental wavelength data in Fig. 77.8(b) represent a series of shots with different interaction beam timings. The error bars on the experimental data represent the statistical variation in the measured wavelength over the compilation of shots. The experimental data of Fig. 77.8(b), translated into the inferred electron densities, are shown as an overlay in
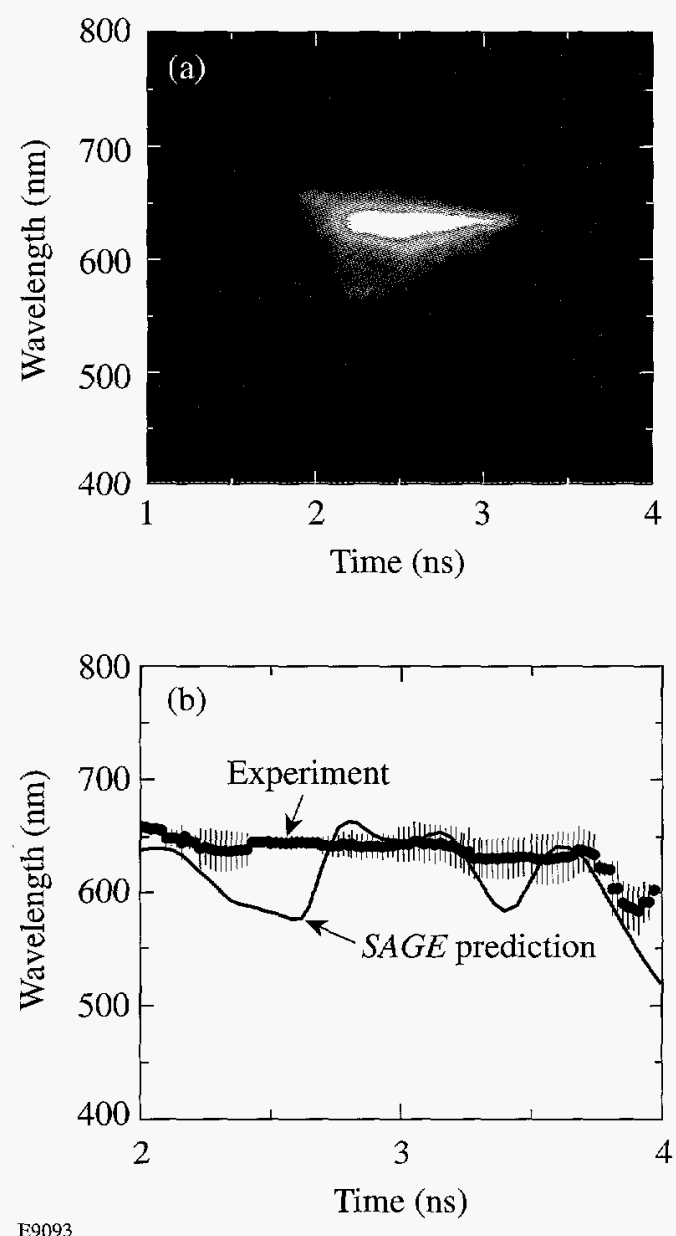

Figure 77.8

(a) Measured SRS spectrum of an exploding-foil plasma with the interaction beam timed to start at 2 ns, and (b) measured peak SRS wavelengths compiled from a series of shots with different interaction beam timings compared with $S A G E$ predictions. The vertical lines associated with the experimental curve in (b) represent variations between different shots.
Fig. 77.5(b). From both figures the agreement between experiment and simulation is seen to be close, and the predicted onset of the drop in density around $3.7 \mathrm{~ns}$ is observed. The oscillations predicted by $S A G E$ are associated with the off-axis mass converging onto the axis in a geometry with cylindrical symmetry. In the experiment the azimuthal asymmetries associated with the finite number of beams in each ring are expected to result in this behavior being averaged out. Both simulation and experiment are consistent with the production of a plasma with the peak $n_{e}$ staying between $0.1 n_{c}$ and $0.2 n_{c}$ for approximately $2 \mathrm{~ns}$.

\section{Laser-Plasma Interaction Experiments}

Signal levels of the direct backscatter of SRS and SBS from the interaction beam were recorded with the experimental setup shown in Fig. 77.9. The backscattered SRS and SBS signals were measured through the focusing lens with a fullaperture pickoff whose front surface was uncoated and whose back surface was AR (sol gel) coated for $351 \mathrm{~nm}$. The backsurface reflectivity in the 400 - to 800 -nm range lies between $2 \%$ and $3 \%$ and is close to the reflectivity of the front surface. This unfortunately degrades the temporal resolution of the SRS measurements to $\sim 250 \mathrm{ps}$ and lengthens the SRS signals by the same amount.

A small fraction of the backscattered light was directed via optical fibers to two visible spectrographs, one near $351 \mathrm{~nm}$ to look for SBS and one covering the 400 - to $700-\mathrm{nm}$ range to look for SRS. Both spectrographs were outfitted with streak cameras. The remainder of the backscattered light was measured with an unfiltered calorimeter (which does not distinguish between SBS and SRS). This calorimeter resolved a small fraction of a joule of light backscattered from the target.

The SRS measurement used a 1/3-m Czerny-Turner spectrograph with $8-\mathrm{nm}$ spectral resolution coupled to a streak camera. A $20-\mathrm{m}, 50-\mu \mathrm{m}$ gradient index fiber transmitted the SRS light from a pickoff at the backscatter station to the entrance slit of the spectrograph. The broad bandwidth of the SRS light necessitated a wavelength-dependent group-velocity dispersion correction, which amounted to $\sim 5 \mathrm{~ns}$ between $351 \mathrm{~nm}$ and $700 \mathrm{~nm}$. This correction was verified by measuring the time delay between the second-order 351-nm signal and a 700-nm SRS/TPD signal. Any error associated with this correction is estimated to be $<50$ ps over this spectral range and consequently negligible. It was found that there was no measurable contribution to the streaked SRS spectra from the secondary beams. 


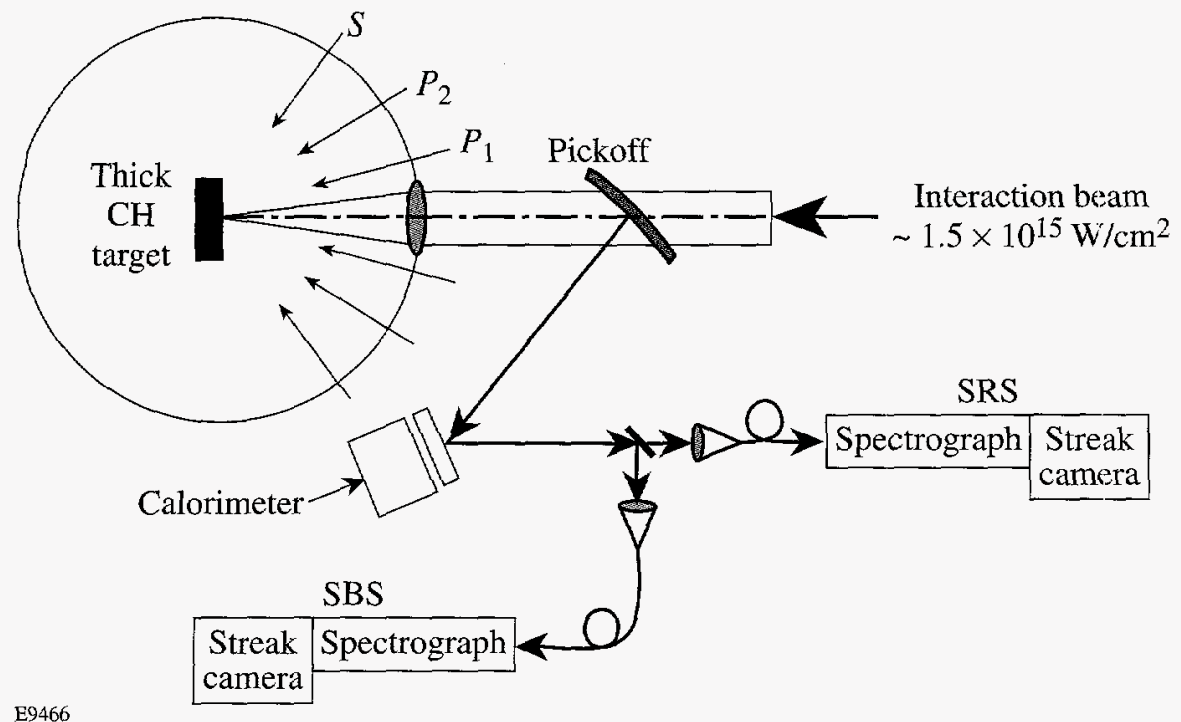

Figure 77.9

Experimental setup used to measure the SRS and SBS transmitted back through the focusing lens. The calorimeter measured the sum of the SRS and SBS energies reflected off the pickoff. Significant SRS reflections were made off each of the pickoff surfaces.

The SBS measurement used a 1-m Czerny-Turner spectrograph with $\sim 0.05$-nm spectral resolution, coupled to a streak camera with $\sim 50$-ps temporal resolution. The background light level entering this spectrograph was measured with shots without the interaction beam. For exploding-foil plasmas the time-resolved backscatter spectra were dominated by scattered light from the primary and secondary beams, and no SBS feature could be identified. Shots with and without the interaction beam at $1.5 \times 10^{15} \mathrm{~W} / \mathrm{cm}^{2}$ were barely distinguishable. (This was in contrast to earlier experiments carried out without a DPP in the interaction beam, where the SBS reflectivity exceeded $10 \%,{ }^{17}$ ) The energy response of the SBS spectrograph was estimated using shots taken without the interaction beam. In this case there was no scattered light in the SRS range, so the energy recorded in the calorimeter corresponded to the signal in the SBS spectrograph.

Measured backscatter SBS spectra are presented in Fig. 77.10 for the exploding-foil plasmas after the peak density has dropped to $\sim n_{c} / 5$. The time during which NIF temperatures and scale lengths are achieved extends from 2 to 3 ns. Figure 77.10(a) shows the temporal evolution of the spectrum near $351 \mathrm{~nm}$ when the interaction beam was not fired and was used to establish the level of background light. The interaction beam was fired at $2 \mathrm{~ns}$ in Fig. $77.10(\mathrm{~b})$ and at $3 \mathrm{~ns}$ in Fig. 77.10 (c). Since there are no significant differences between the three images, it is clear that no measurable contribution from the interaction beam to the signal near $351 \mathrm{~nm}$ was observed for either timing. The energy response of the spectrograph places an upper limit on the SBS reflectivity of $0.1 \%$ for these experiments.
A similar set of streaked spectra is shown in Fig. 77.11 for solid-target plasmas. The signal near $t=0$ in all three cases is interpreted as light reflected from the critical surface with a Doppler blue shift from the expanding plasma. This is consistent with the $S A G E$ prediction for the time-dependent absorbed power [Fig. 77.3(b)]. Less signal is seen during the second set of primary beams, as they were incident at greater angles from the collection lens. The feature around $351 \mathrm{~nm}$ in Fig. 77.11(b) between 2 and 3 ns corresponds to a $0.1 \%$ reflection of the interaction beam from near the critical-density surface. The total energy recorded by the spectrograph was $3.1 \mathrm{~J}$, corresponding to $0.7 \%$ of the interaction beam energy, so most of the energy was in the scattered $P_{1}$ beams. The feature after $3 \mathrm{~ns}$ in Fig. 77.11(c) is from what would, in the absence of the interaction beam, be a rapidly cooling plasma. This plasma, which still has a critical-density surface, is strongly absorbing (close to $100 \%$ absorption is predicted) and is locally heated by the interaction beam. The observed backscatter is consistent with a backscattered SBS energy fraction of $0.2 \%$. Thus, no significant SBS was observed in either the exploding-foil or solid-target interaction experiments under NIF directdrive conditions.

Small amounts of SRS were observed in both types of plasma. For the exploding-foil plasmas SRS from the electrondensity maximum was used as a density diagnostic (see preceding section). For solid-target plasmas, time-resolved SRS was measured through the focusing lens (direct backscatter) and at $20^{\circ}$ from the backscatter direction (Fig. 77.12). Figures 77.12(a) and 77.12(b) show the temporal evolution of the SRS backscatter spectra through the lens, with the interaction 
beam fired at $2 \mathrm{~ns}$ and $2.5 \mathrm{~ns}$, respectively, with peak intensity $1.5 \times 10^{15} \mathrm{~W} / \mathrm{cm}^{2}$. Both images exhibit broad spectral features coincident in time with the interaction beam and extending from $420 \mathrm{~nm}$ to $540 \mathrm{~nm}$. The weak feature at $700 \mathrm{~nm}$ appears to be $\omega_{0} / 2$ light from the two-plasmon decay instability driven by the primary and secondary beams.

An upper limit on the backscattered SRS energy can be estimated from the calorimetry and the SBS signal. As mentioned above, the spectral energy response of the SBS spectrograph was determined on shots where the interaction beam was not fired. By subtracting the SBS energy in the spectrograph from the total energy recorded in the calorimeter, the

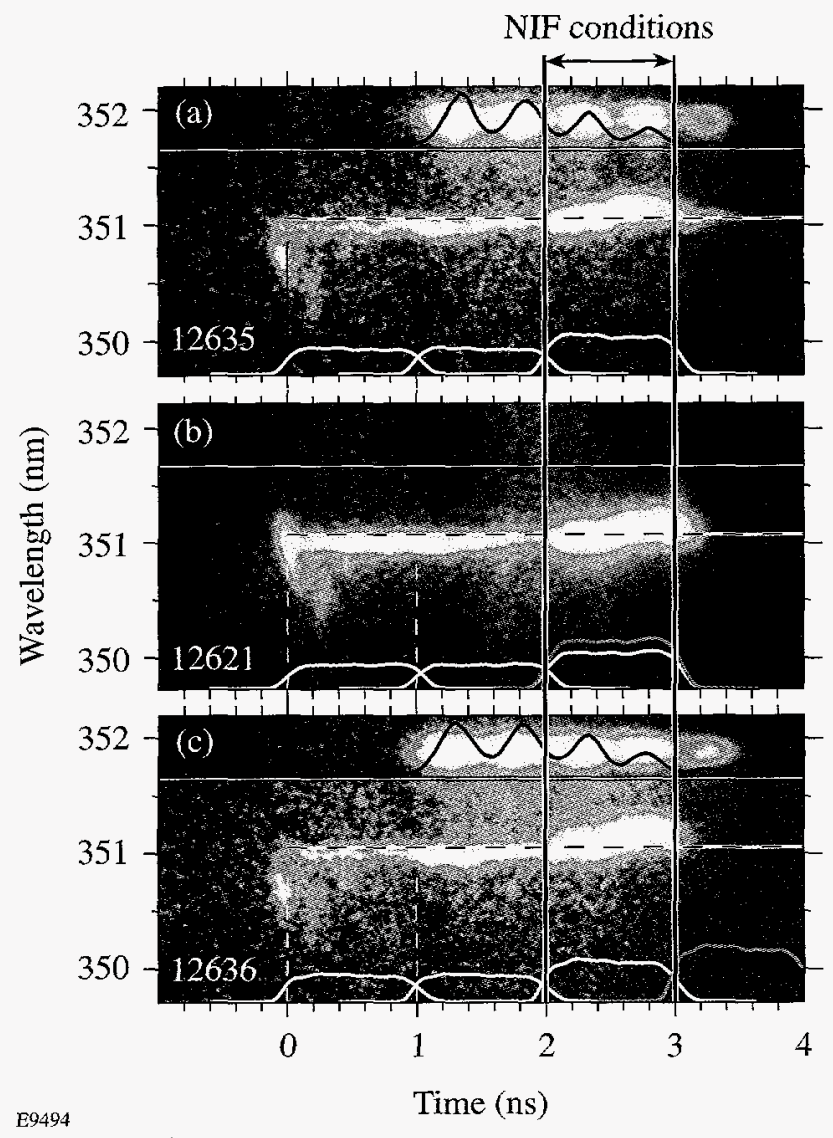

Figure 77.10

Streaked spectra around $351 \mathrm{~nm}$ of light backscattered from exploding-foil plasmas through the interaction-beam focus lens for (a) no interaction beam, (b) interaction beam at $2 \mathrm{~ns}$, and (c) interaction beam at $3 \mathrm{~ns}$. A multipulse timing fiducial [shown at the top of (a) and (c) with the solid line indicating its temporal shape] enabled an absolute time origin to be assigned to the backscattered light. The measured streak records of the four groups of laser beams are also shown. The horizontal dashed line indicates the unshifted laser wavelength. energy in the SRS signal can in principle be estimated. Due to the shot-to-shot fluctuations in the signal levels, however, there is a large uncertainty associated with this subtraction. In addition, the final turning mirror has $\sim 100 \%$ reflectivity at $351 \mathrm{~nm}$ but a flat $\sim 12 \%$ reflectivity between 400 and $700 \mathrm{~nm}$, further reducing the SRS contribution in the calorimeter. Taking all these factors into account, the residual SRS energy in the calorimeter is $0 \mathrm{~J}$ with an uncertainty of $25 \mathrm{~J}$. The upper limit of $25 \mathrm{~J}$ corresponds to a maximum SRS backscattered energy fraction of $5 \%$, but clearly a lower value is more likely. An accurate measurement of the SRS fraction will require further experiments.

Landau damping is very strong in this density-temperature regime $\left(k \lambda_{\mathrm{D}} \approx 0.5\right.$ at $570 \mathrm{~nm}$ during the high-temperature portion of the interaction), which suggests that the observed SRS originates in filaments where Landau damping is strongly reduced. ${ }^{18}$

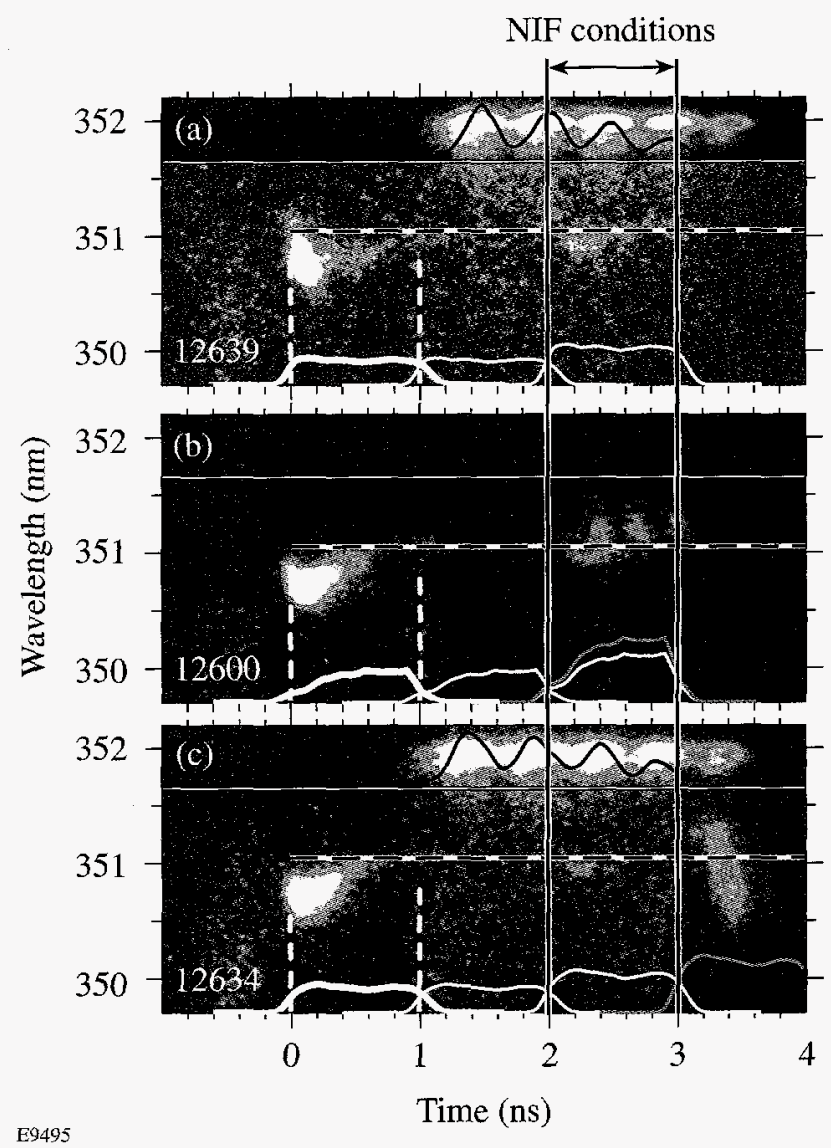

Figure 77.11

Same as Fig. 77.10 but for the solid targets (partial spheres of 2-mm diameter). 


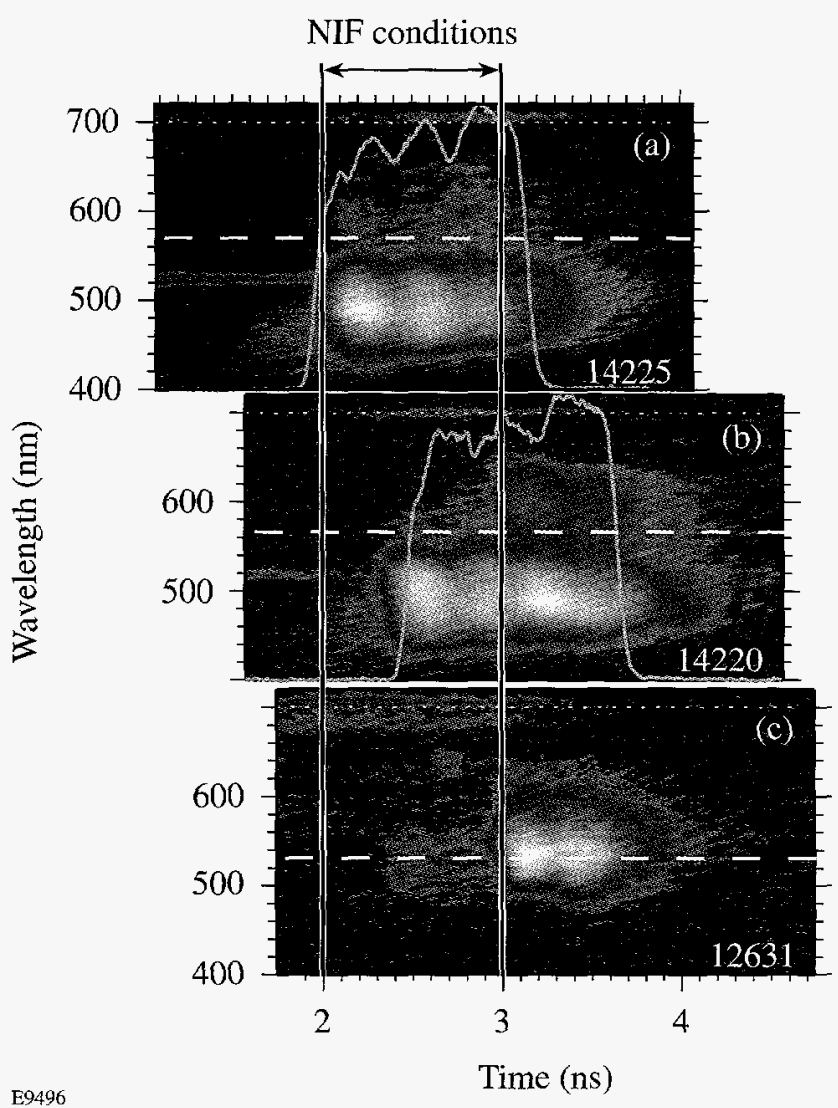

Figure 77.12

Streaked SRS spectra from solid-target plasmas: (a) and (b) directly back through the lens, with SSD on (0.25-THz UV bandwidth), and (c) at $20^{\circ}$ to the lens with SSD off. The interaction beam was timed at $2 \mathrm{~ns}$ in (a) and at $2.5 \mathrm{~ns}$ in (b) and (c). The temporal shape of the interaction beam is shown as an overlay in (a) and (b). The targets were flat in (a) and (b) and a partial sphere of $2-\mathrm{mm}$ diameter in (c).

A time-resolved sidescattered SRS spectrum from a solid target, at $20^{\circ}$ from the interaction beam direction (the normal to the original target surface), is shown in Fig. 77.12(c) with the interaction beam fired at $2.5 \mathrm{~ns}$. No SRS is observed in sidescattering during the hot NIF-like plasma phase between $t=2$ and $3 \mathrm{~ns}$, but sidescattering sets in abruptly when the secondary beams turn off at $t=3 \mathrm{~ns}$. At this time the background plasma cools down, although this alone may not explain the abrupt onset of sidescattering. The sidescatter SRS appears at longer wavelengths (i.e., higher densities) than the filamentary SRS seen in backscattering between 2 and 3 ns. This is consistent with the onset of SRS at longer wavelengths in direct backscattering at $3 \mathrm{~ns}$ [Fig. 77.12(b)].
It is possible that some SRS was present just outside the $f / 6$ cone of the interaction-beam focusing lens; however, this is unlikely to be significant, based on the lack of SRS at $20^{\circ}$ from 2 to $3 \mathrm{~ns}$.

Combining the backscatter and sidescatter SRS observations it is reasonable to conclude that the directionality of the filamentary (low-density) SRS signal is due to the "horn antenna" effect, which directs the incident 351-nm light into the filaments and then funnels the backscattered SRS signal through the same horn to the focusing lens. Within the filament the SRS signal is guided by the filament as well as by the SRS gain, while in the region of the horn geometrical optics is applicable and the backscattered SRS light is guided by the density structure making up the horn. It is impossible to determine the exact background density within which the filaments are created since the SRS wavelength merely reflects the density within the filaments. It is clear that the 2-D SSD bandwidth used in this experiment $(0.25 \mathrm{THz}$ in the UV) is insufficient to suppress the filamentation.

The density-gradient threshold for Raman scattering (e.g., that given by $\mathrm{Kruer}^{4}$ ) yields $I \sim 1.8 \times 10^{15} \mathrm{~W} / \mathrm{cm}^{2}$, and SRS would be expected to originate within the speckles produced by the phase plates. During the hot NIF-like plasma phase, however, Landau damping is strong enough to suppress this SRS completely, while in the cooling phase (past $t=3 \mathrm{~ns}$ ) SRS can occur and is seen between 0.1 and $0.2 n_{c}$. This type of Raman scattering (without filamentation) is still preferentially directed in the backward direction, but it is much less collimated, which thus allows its observation through the lens as well as at $20^{\circ}$ away from the backscatter direction.

\section{Conclusion}

Long-scale-length plasmas, with parameters relevant to the peak of direct-drive NIF laser pulses, have been produced and characterized on the OMEGA laser system. Temperatures of $\sim 4 \mathrm{keV}$ and densities of $\sim 0.2 n_{c}$ have been measured in agreement with hydrocode predictions and are consistent with density scale lengths of $\sim 0.5$ to $1.0 \mathrm{~mm}$. Experiments have shown that these plasmas have an SBS reflectivity of less than $0.1 \%$ and an SRS reflectivity of less than $\sim 5 \%$, even when the interaction beam intensity is $1.5 \times 10^{15} \mathrm{~W} / \mathrm{cm}^{2}$, eight times higher than the NIF cluster intensity. The measured backscattered SRS signals are believed to originate in filaments at $n_{e}<0.1 n_{\mathcal{C}}$, as otherwise they would be suppressed by the strong Landau damping that occurs at the measured temperatures. The threshold intensity for SRS was not determined since experiments were not performed with a lower- 
intensity interaction beam. The calculated SRS and SBS intensity thresholds were comparable to the intensity of the interaction beam at $1.5 \times 10^{15} \mathrm{~W} / \mathrm{cm}^{2}$; however, only SRS was observed in the experiment.

On the basis of these experiments it is concluded that SRS and SBS are not likely to present a problem for the coronal plasmas at the peak of the NIF direct-drive laser pulse. Future experiments on OMEGA will vary the intensity of the interaction beam and will address the parametric instabilities of the coronal plasmas in the foot and transition regions of the NIF direct-drive laser pulse.

\section{ACKNOWLEDGMENT}

The authors thank Dr. C. P. Verdon for providing the predicted NIF profiles and T. Kessler and D. Smith for the design and fabrication of the DPP's used in these experiments. This work was supported by the U.S. Department of Energy Office of Inertial Confinement Fusion under Cooperative Agreement No. DE-FC03-92SF19460, the University of Rochester, and the New York State Energy Research and Development Authority. The support of DOE does not constitute an endorsement by DOE of the views expressed in this article.

\section{REFERENCES}

1. C. P. Verdon, Bull. Am. Phys. Soc. 38, 2010 (1993).

2. S. E. Bodner, D. G. Colombant, J. H. Gardner, R. H. Lehmberg, S. P. Obenschain, L. Phillips, A. J. Schmitt, J. D. Sethian, R. L. McCrory, W. Seka, C. P. Verdon, J. P. Knauer, B. B. Afeyan, and H. T. Powell, Phys. Plasmas 5, 1901 (1998)

3. J. D. Lindl, Phys. Plasmas 2, 3933 (1995).

4. W. L. Kruer, The Physics of Laser Plasma Interactions, Frontiers in Physics, Vol. 73, edited by D. Pines (Addison-Wesley, Redwood City, CA, 1988).

5. J. C. Fernández et al., Phys. Plasmas 4, 1849 (1997).
6. B. J. MacGowan et al., Phys. Plasmas 3, 2029 (1996).

7. T. R. Boehly, D. L. Brown, R. S. Craxton, R. L. Keck, J. P. Knauer, J. H. Kelly, T. J. Kessler, S. A. Kumpan, S. J. Loucks, S. A. Letzring, F. J. Marshall, R. L. McCrory, S. F. B. Morse, W. Seka, J. M. Soures, and C. P. Verdon, Opt. Commun. 133, 495 (1997).

8. Y. Lin, T. J. Kessler, and G. N. Lawrence, Opt. Lett. 21, 1703 (1996).

9. R. S. Craxton and R. L. McCrory, J. Appl. Phys. 56, 108 (1984).

10. M. C. Richardson, P. W. McKenty, F. J. Marshall, C. P. Verdon, J. M. Soures, R. L. McCrory, O. Barnouin, R. S. Craxton, J. Delettrez, R. L. Hutchison, P. A. Jaanimagi, R. Keck, T. Kessler, H. Kim, S. A. Letzring, D. M. Roback, W. Seka, S. Skupsky, B. Yaakobi, S. M. Lane, and S. Prussin, in Laser Interaction and Related Plasma Phenomena, edited by H. Hora and G. H. Miley (Plenum Publishing, New York, 1986), Vol. 7, pp. 421-448.

11. W. Seka, R. S. Craxton, R. E. Bahr, D. L. Brown, D. K. Bradley, P. A. Jaanimagi, B. Yaakobi, and R. Epstein, Phys. Fluids B 4, 432 (1992).

12. S. Skupsky, R. W. Short, T. Kessler, R. S. Craxton, S. Letzring, and J. M. Soures, J. Appl. Phys. 66, 3456 (1989).

13. D. H. Kalantar et al., in 22nd International Congress on High-Speed Photography and Photonics, edited by D. L. Paisley and A. M. Frank (SPIE, Bellingham, WA, 1997), Vol. 2869, pp. 680-685.

14. Available from Cascade Applied Sciences, Inc., P.O. Box 4477, Boulder, $\mathrm{CO} 80306$

15. R. W. Lee, B. L. Whitten, and R. E. Stout II, J. Quant. Spectrosc. Radiat. Transfer 32, 91 (1984).

16. R. S. Marjoribanks, M. C. Richardson, P.A. Jaanimagi, and R. Epstein, Phys. Rev. A 46, 1747 (1992).

17. A. V. Chirokikh, R. S. Craxton, D. D. Meyerhofer, A. Simon, W. Seka, and R. P. Drake, Bull. Am. Phys. Soc. 42, 1882 (1997).

18. R. W. Short and A. Simon, Phys. Plasmas 5, 4134 (1998). 


\section{Irradiation Uniformity for High-Compression Laser-Fusion Experiments}

\begin{abstract}
Various techniques are being used to achieve the high irradiation uniformity required for direct-drive, laser-fusion experiments on the OMEGA laser system. These techniques are directly applicable to the National Ignition Facility (NIF) being built at the Lawrence Livermore National Laboratory. The combination of two-dimensional smoothing by spectral dispersion (SSD), ${ }^{1-3}$ distributed phase plates (DPP's), ${ }^{4,5}$ polarization smoothing (DPR's), ${ }^{6,7}$ and beam overlap should be sufficient to reach the rms level of $1 \%$ or less when the laser intensity has been averaged over a few hundred picoseconds. Of these, SSD is the dominant mechanism for reducing the nonuniformity.
\end{abstract}

The SSD beam-smoothing technique produces uniform laser beams in a time-averaged sense. The level of uniformity that can be achieved is determined by two factors: bandwidth and spectral dispersion. The amount of bandwidth determines the rate of smoothing, and the amount of spectral dispersion determines the level of uniformity that can be achieved (as well as the longest spatial wavelength of nonuniformity that can be smoothed). Frequency-tripled glass lasers (such as OMEGA and the NIF) place constraints on both bandwidth and spectral dispersion. Until recently, high-efficiency frequency tripling of laser light was limited to a bandwidth of $3 \AA$ to $4 \AA$ in the IR. Recent calculations and experiments ${ }^{8,9}$ have shown that this bandwidth can be increased by a factor of 3 to 4 by using a second tripling crystal, resulting in $\sim 1 \mathrm{THz}$ in the UV, with a $\sim 70 \%$ tripling efficiency. Second triplers will be installed on OMEGA during 1999.

The spectral dispersion of the bandwidth on OMEGA has been limited by the laser spatial-filter pinholes to an angular spread of $\sim 50 \mu \mathrm{rad}$ (relative to the output of the system). This will be increased on OMEGA during 1999 to accommodate asymmetric SSD dispersion, with $100 \mu \mathrm{rad}$ in one direction and $50 \mu \mathrm{rad}$ in the other. Polarization smoothing will provide an additional $50 \mu \mathrm{rad}$, resulting in a total angular spread of $100 \mu \mathrm{rad}$ in each direction. ${ }^{10}$ With these laser modifications, it is expected that the levels of uniformity required for highcompression experiments on OMEGA will be achieved. The rms nonuniformity will drop below $1 \%$ after a smoothing time of $250 \mathrm{ps}$. These same uniformity techniques are directly applicable to the NIF and will result in even higher levels of uniformity because of the larger number of beams (192 versus 60).

With the angular divergence of the beam that will be achieved on OMEGA during 1999, all spatial wavelengths of nonuniformity of concern for ICF will be smoothed. The longest wavelength of nonuniformity that can be smoothed by SSD and polarization smoothing can be estimated in the following way: Both SSD and DPR's smooth nonuniformities by shifting the speckle pattern produced by a phase plate. Two overlapped speckle patterns that have been shifted by a distance $S$ will exactly smooth a nonuniformity of spatial wavelength $2 S$. The maximum speckle shift is given by $S_{\max }=F \Delta \theta$, where $F$ is the focal length and $\Delta \theta$ is the beam angular divergence; thus, SSD can smooth spherical harmonic modes of nonuniformity down to $\ell_{\text {cut }}=2 \pi R /\left(2 S_{\max }\right)$, where $R$ is the target radius. Using OMEGA parameters $F=180 \mathrm{~cm}, R=$ $500 \mu \mathrm{m}$, and $\Delta \theta=90 \mu \mathrm{rad}$, we have $\ell_{\mathrm{cut}} \sim 10$, which is well below the modes of concern for seeding hydrodynamic instabilities. For the NIF, the same angular divergence will produce essentially the same value of $\ell_{\text {cut }}$ since both the focal length and the target radius will be 3.5 to 4 times larger than for OMEGA.

The mathematical formalism describing 2-D SSD is presented in Ref. 2. We can use the approximate asymptotic expression for the SSD reduction factor [Eq. (20) in Ref. 2] to confirm the above estimates for the longest wavelength of nonuniformity that can be smoothed by SSD. First, this equation is modified to include the contribution of polarization smoothing as follows: Let the polarization dispersion be in the $y$ direction, with $\Delta_{p}$ the spatial separation between $e$ and $o$ rays in the target plane. Then the superposition of the $e$ and $o$ intensities is $\frac{1}{2}\left[I\left(x, y+\Delta_{p} / 2\right)+I\left(x, y-\Delta_{p} / 2\right)\right]$. For the 
asymptotically smoothed intensity, the SSD reduction factor $R_{\mathrm{ij}}^{p}$ is

$$
\begin{aligned}
R_{\mathrm{ij}}^{p}= & J_{0}\left(6 \delta_{1} \sin \frac{1}{2} k_{i} \Delta_{1}\right) \times J_{0}\left(6 \delta_{2} \sin \frac{1}{2} k_{j} \Delta_{2}\right) \\
& \times \cos \left(\frac{1}{2} k_{j} \Delta_{p}\right)
\end{aligned}
$$

for spatial modes $\left(k_{i}, k_{j}\right)$ in the $(x, y)$ directions. The factor $6 \delta_{1,2}$ is the number of spectral modes for each direction of SSD (after frequency tripling), and $\Delta_{1,2}$ is the separation of the modes. The first zero of the first Bessel function will determine the longest wavelength for which there is substantial smoothing in the $x$ direction. Expanding the "sin" function for small $k$ and using $S_{1}=6 \delta_{1} \Delta_{1}$ as the maximum spectral shift, the argument of the Bessel function becomes $\frac{1}{2} \ell S_{1} / R$, where $\ell$ is the effective spherical harmonic mode number $(\ell=k \times R)$. This gives $\ell_{\text {cut }}=15$, where $J_{0}\left(\frac{1}{2} \ell_{\text {cut }} S_{1} / R\right)=0$, which is a little higher than the rough estimate above.

In the perpendicular direction, there is smoothing from both the spectral dispersion and the polarization spread. For the strategy being implemented on OMEGA, the spectral angular spread in this direction is half the spread in the other direction $\left(S_{2}=\frac{1}{2} S_{1}\right)$ in order to keep the laser pinholes as small as possible. The total speckle shift in this direction is then doubled by means of the polarization shift for $\Delta_{p}=S_{2}$. With these parameters, the "cos" term in Eq. (1) gives the lowest value of $\ell$ for which $R$ is zero in the $y$ direction, namely $\ell \approx 20$. Of course there is still some smoothing below $\ell=20$.

The smoothing of long-wavelength nonuniformities can be seen in the full time-dependent calculation shown in Fig. 77.13. Shown is the calculated rms nonuniformity for 60 overlapping beams on a spherical target (using the OMEGA irradiation geometry). The nonuniformity for each individual beam was calculated from the time-dependent SSD equations in Ref. 2, and the result was projected onto the sphere. The first image shows how the nonuniformity spectrum has decreased after $500 \mathrm{ps}$ of smoothing for 500 modes, with $1 \mathrm{THz}$ of bandwidth. [Plotted is the rms nonuniformity $\sigma_{\ell}$ of the spherical harmonic mode $\ell$, defined such that the total rms nonuniformity is $\left(\Sigma \sigma_{\ell}^{2}\right)^{1 / 2}$. The second image shows the spectrum for the first 50 modes, which is a region of particular concern for direct-drive laser fusion.

We first note that even without SSD, the nonuniformity in the very low-order modes ( $\ell=11$ to 30$)$ is very small, $1 \%$. After 500 ps of smoothing, this has been reduced to $0.15 \%$. Smoothing is occurring for modes that are about a factor of 2 lower than indicated in the results of Rothenberg. ${ }^{3}$ The reason is that this calculation has used twice the angular divergence of Rothenberg; he limited the angular spread of the beam to be $50 \mu \mathrm{rad}$. We have increased the spread to $100 \mu \mathrm{rad}$ in one direction and doubled the 50- $\mu \mathrm{rad}$ spread in the second direc-
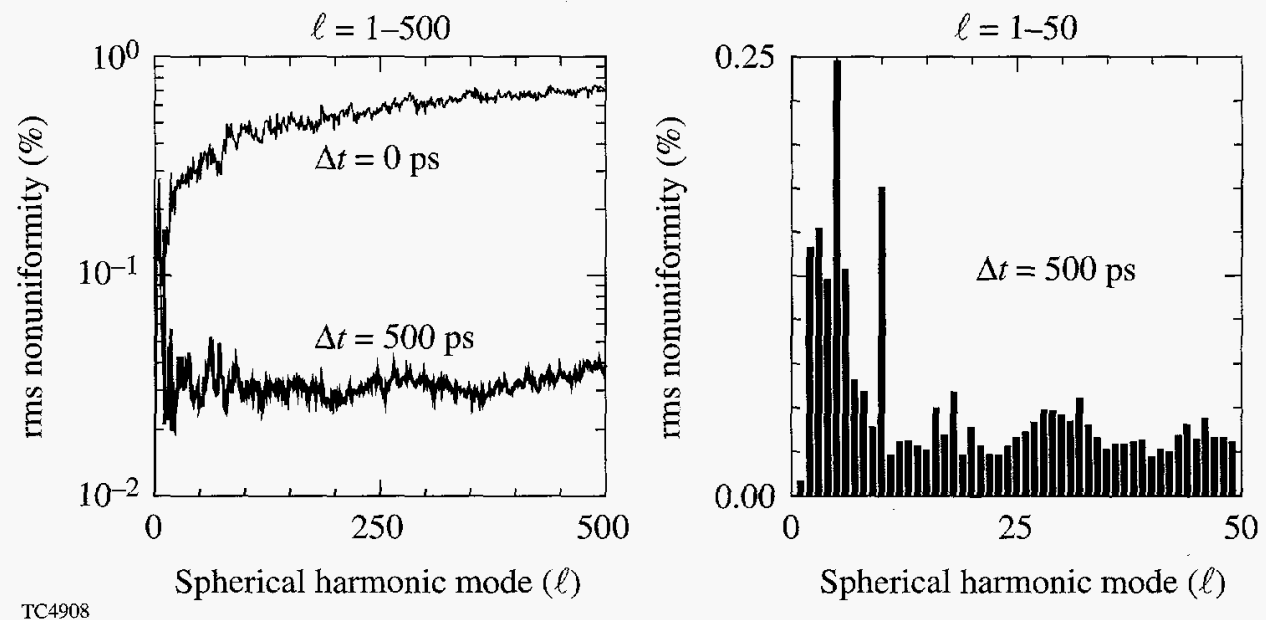

Figure 77.13

The reduction in nonuniformity produced by SSD on OMEGA when the intensity is averaged over $500 \mathrm{ps,} \mathrm{for} \mathrm{a} \mathrm{bandwidth} \mathrm{of} 1 \mathrm{THz}$ and a total angular spread of $100 \mu \mathrm{rad}$. The IR bandwidths for the two modulators are $2.1 \AA$ and $10.2 \AA$ with modulation frequencies of $8.8 \mathrm{GHz}$ and $10.4 \mathrm{GHz}$. 
tion by means of the polarization wedge. This is consistent with the above analysis showing that when the total speckle shift is about $15 \%$ of the target diameter, modes below $\ell=15$ are smoothed. These calculations have included the effect that the envelope, as well as the speckle, is shifted. The smoothing effect is more dramatic for higher-order modes; for $\ell=31$ to 500 the nonuniformity is reduced from $13 \%$ to $0.6 \%$ rms.

The second image in Fig. 77.13 shows details of the longwavelength nonuniformity structure after 500 ps of smoothing (note that the vertical scale has changed). It is now more clearly seen that modes down to $\ell=11$ have been smoothed. The spike at $\ell=10$ as well as the additional nonuniformity at lower $\ell$ is the result of how the 60 beams overlap on the spherical target; it is not the result of structure on an individual beam. This form of nonuniformity can be reduced by a careful choice of the radial beam profile that is generated by the phase plate. These calculations have used the $\operatorname{sinc}^{2}$ profile that is generated by square phase-plate elements (and modified by SSD), with the target boundary near the $5 \%$ intensity contour.

The discussion after Eq. (1) shows that the asymptotic level of smoothing reached by SSD in the low-order modes depends only on the size of the speckle deflections: $S_{1}, S_{2}$, and $\Delta_{p}$. (This is valid over the range of wavelengths for which the "sin" functions in that equation can be expanded to first order.) The amount of time required to reach this level can be decreased, however, by increasing the number of color cycles. ${ }^{1-3}$ This occurs because the longer wavelengths of nonuniformity are produced by interference between phase-plate elements that are relatively close together. By increasing the number of color cycles, the relative phase between close phase-plate elements varies more rapidly, and faster smoothing occurs. This is at the expense, however, of reduced smoothing for very short wavelengths of nonuniformity (high $\ell$ ) produced by interference between more-distant phase-plate elements.

One example of the effect of three color cycles compared to one cycle is shown in Fig. 77.14. This corresponds to an intermediate case that might be examined on OMEGA before the implementation of $1 \mathrm{THz}$ of bandwidth. Both cases in Fig. 77.14 correspond to a bandwidth of $0.3 \mathrm{THz}$ with one color cycle and a polarization wedge in one of the directions. In the second direction, one case has one color cycle and the other has three, produced by a threefold increase in modulation frequency. The results are given after $250 \mathrm{ps}$ of smoothing. (The total nonuniformity is about $1.7 \%$ rms.) For three color cycles, the nonuniformity in modes 50 to 200 has been reduced by about $50 \%$, at the expense of some increased nonuniformity

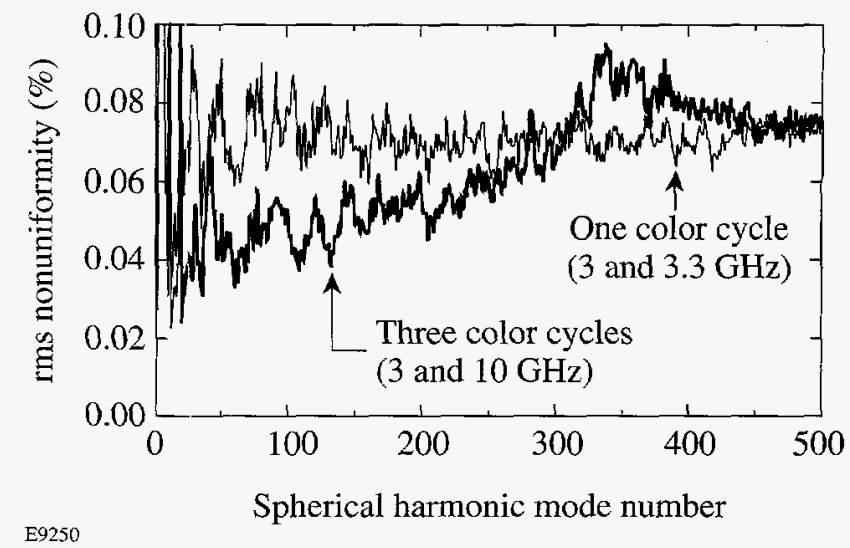

Figure 77.14

By increasing the number of color cycles, long wavelengths of nonuniformity can be reduced at a faster rate. The nonuniformity spectrum with $0.3 \mathrm{THz}$ of bandwidth and 250 ps of smoothing time is compared for one and three color cycles. The bancwidths in both cases were $1.5 \times 3 \AA$. For one color cycle the modulation frequencies were 3 and $3.3 \mathrm{GHz}$. For three color cycles (in one direction) the $3.3-\mathrm{GHz}$ modulator was replaced by a $10-\mathrm{GHz}$ modulator.

above $\ell=300$. It is believed that this shift in the nonuniformity spectrum is beneficial, as modes below $\ell=200$ are considered to be the most dangerous. After about 1 ns of smoothing time, however, both examples show about the same asymptotic level of nonuniformity for the long wavelengths.

Finally, in Fig. 77.15 we compare the effects of the different improvements in uniformity that are planned for OMEGA during 1999. The current SSD configuration consists of IR bandwidths of $1.25 \times 1.75 \AA$ with electro-optic (EO) modulators of 3 and $3.3 \mathrm{GHz}$. The spectral divergence is less than $50 \mu \mathrm{rad}$. A small number of polarization wedges are available for planar experiments. A full set of 60 wedges will be installed during 1999 for spherical experiments. At the same time, the bandwidth will be increased to $1.5 \times 3.0 \AA$ with the resulting spectral/polarization dispersion being $\sim 100 \mu \mathrm{rad}$ in each direction. During the latter half of the year, one of the EO modulators will be replaced by a $10-\mathrm{GHz}$ modulator and the IR bandwidth increased to $\sim 12 \AA$, resulting in a UV bandwidth of $\sim 1 \mathrm{THz}$.

The improvements in irradiation nonuniformity planned for the OMEGA laser during 1999 will reduce the rms nonuniformity to less than $1 \%$ when the intensity is averaged over $300 \mathrm{ps}$. The total nonuniformity in the long-wavelength nonuniformities (spherical harmonic modes 11 to 30 ) can be smoothed to levels below $0.15 \%$. This is being accomplished by the addition of three new features to the laser: (1) Second 


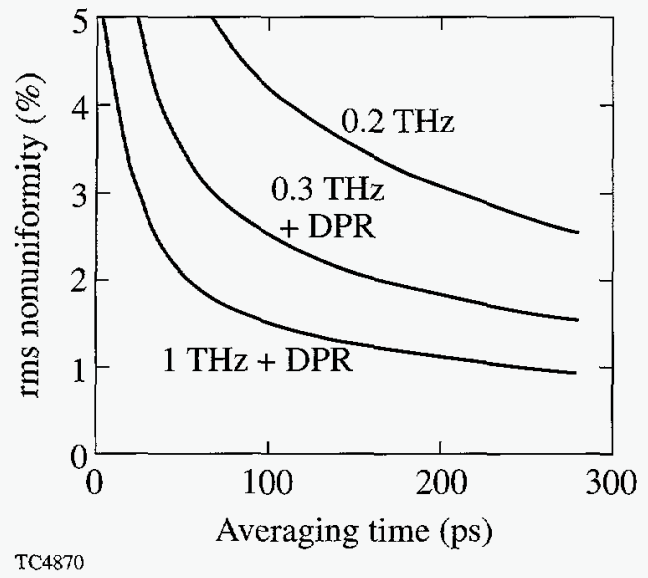

Figure 77.15

The rms nonuniformity as a function of time for the current SSD configuration on OMEGA and the upgrades planned for 1999 , including the effect of multiple-beam overlap on a spherical target in the 60-beam geometry. Spherical harmonic modes up to $\ell=500$ have been considered with no additional smoothing assumed in the plasma atmosphere around the target. The top curve shows the current level of irradiation uniformity. The middle curve corresponds to a higher bandwidth in one direction and the inclusion of polarization smoothing. The bottom curve is the result for $1 \mathrm{THz}$ of UV bandwidth and polarization smoothing.

triplers will allow the high-efficiency tripling of 3 to 4 times the current bandwidth. This will decrease the smoothing time by the same factor. (2) Polarization wedges added to all 60 beams will instantaneously reduce nonuniformity by $\sqrt{2}$ and double the maximum shift of speckle patterns on the target. (3) An increase in laser-pinhole size will double the allowed spectral spread in one direction from $50 \mu \mathrm{rad}$ to $100 \mu \mathrm{rad}$. (The angular spread in the perpendicular direction will remain $50 \mu \mathrm{rad}$.) The combined effect of the polarization spread and the increased spectral spread will be a factor-of-2 reduction in the largest spherical harmonic mode of nonuniformity that can be smoothed, and a reduction in the asymptotic value of nonuniformity by almost the same factor.

These uniformity improvements are directly applicable to the NIF. As with OMEGA, the current pinhole specification for the NIF limits the angular spread of the beam to $50 \mu \mathrm{rad}$. The NIF optical design should be examined to determine if the pinhole can be opened further for direct-drive experiments as is being done on OMEGA. Using essentially the same SSD configuration as OMEGA, including polarization smoothing and dual-tripler frequency conversion, even higher uniformity will be achievable on the NIF due to the larger number of beams (192 versus 60). A comparison between NIF and

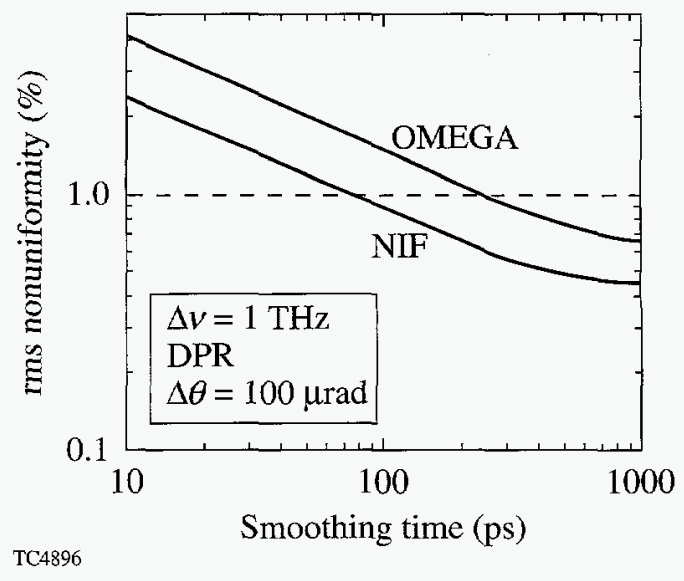

Figure 77.16

The rms nonuniformity as a function of time for the NIF and for OMEGA, assuming 1-THz bandwidth and polarization smoothing. All spherical harmonic modes between 5 and 500 are included.

OMEGA uniformity is shown in Fig. 77.16. It is expected that this smoothing rate and the level of uniformity should be adequate for direct-drive ignition experiments.

\section{ACKNOWLEDGMENT}

This work was supported by the U.S. Department of Energy Office of Inertial Confinement Fusion under Cooperative Agreement No. DE-FC0392SF19460, the University of Rochester, and the New York State Energy Research and Development Authority. The support of DOE does not constitute an endorsement by DOE of the views expressed in this article.

\section{REFERENCES}

1. S. Skupsky, R. W. Short, T. Kessler, R. S. Craxton, S. Letzring, and J. M. Soures, J. Appl. Phys. 66, 3456 (1989).

2. Laboratory for Laser Energetics LLE Review 69, 1, NTIS document No. DOE/SF/19460-152 (1996). Copies may be obtained from the National Technical Information Service, Springfield, VA 22161; S. Skupsky and R. S. Craxton, "Irradiation Uniformity for HighCompression Laser Fusion Experiments," to be published in Physics of Plasmas.

3. J. E. Rothenberg, J. Opt. Soc. Am. B 14, 1664 (1997).

4. T. J. Kessler, Y. Lin, J. J. Armstrong, and B. Velazquez, in Laser Coherence Control: Technology and Applications, edited by H. T. Powell and T. J. Kessler (SPIE, Bellingham, WA, 1993), Vol. 1870, p. 95.

5. Y. Lin, T. J. Kessler, and G. N. Lawrence, Opt. Lett. 21, 1703 (1996).

6. Y.Kato, unpublished notes (1984); K. Tsubakimoto etal, Opt. Commun. 91, 9 (1992); K. Tsubakimoto et al., Opt. Commun. 103, 185 (1993). 
7. Laboratory for Laser Energetics LLE Review 45, 1, NTIS document No. DOE/DP40200-149 (1990). Copies may be obtained from the National Technical Information Service, Springfield, VA 22161; T. E. Gunderman, J.-C. Lee, T. J. Kessler, S. D. Jacobs, D. J. Smith, and S. Skupsky, in Conference on Lasers and Electro-Optics, Vol. 7, 1990 OSA Technical Digest Series (Optical Society of America, Washington, DC, 1990), p. 354.

8. D. Eimerl et al., Opt. Lett. 22, 1208 (1997).
9. A. Babushkin, R. S. Craxton, S. Oskoui, M. J. Guardalben, R. L. Keck, and W. Seka, Opt. Lett. 23, 927 (1998).

10. R. S. Craxton and S. Skupsky, Bull. Am. Phys. Soc. 40, 1826 (1995).

11. The description of the SSD formalism here is similar to the presentation in the internal report of Ref. 2.

12. Y. Kato et al., Phys. Rev. Lett. 53, 1057 (1984). 


\section{A Novel Charged-Particle Diagnostic for $\rho R$ in Compressed ICF Targets}

Areal density - the product of density and thickness of specific ions $(\rho R)$ in ICF targets-is an important measure of compression that enables a comparison of ICF implosions with simulation. In particular, this quantity influences several crucial aspects of an igniting target: the degree of self-heating in the target, its fractional burn, and gain. ${ }^{1}$

Several methods involving nuclear reaction products have been employed to deduce this quantity in ICF implosions. ${ }^{2-5}$ In this article, we will discuss the use of knock-on particles (deuterons and protons) that have been elastically scattered from the $14-\mathrm{MeV}$ primary DT fusion neutrons. Both knock-on deuterons ${ }^{2}$ and protons ${ }^{5}$ have been discussed previously in the literature. The knock-on deuteron diagnostic has been used extensively to provide a measure of $\rho R .^{2}$ The number of these knock-on particles can provide information on the areal densities of the layer in which they are produced, and the energy loss of these particles as they propagate out of the target will provide additional information about $\rho R$ along the propagation path. The deuteron diagnostic can provide information about the compressed target in a relatively model-independent way for values of target $\rho R$ up to $\sim 100 \mathrm{mg} / \mathrm{cm}^{2}$. For higher values of $\rho R$, the knock-on spectrum is significantly distorted and becomes very dependent on temperature within the target.

Maximum information from the knock-on diagnostics is obtained using detailed information about the shape and magnitude of the knock-on spectrum. Until recently, the technique used to detect the knock-on deuterons has involved the counting of tracks satisfying selective criteria in stacked track detectors, consequently providing only gross information about the particle spectrum. This lack of spectral information has limited the use of knock-on ions as a diagnostic. With the deployment of the new charged-particle spectrometer ${ }^{6}$ (CPS), detailed spectral information of charged reaction products from the imploding target can now be obtained, enabling a more detailed analysis of conditions in the target using the knock-on particles. Using a 7.5-kG magnet, the CPS can momentum-select incident charged particles, which are then impinged onto a detector plane consisting of track detectors.
The identity of each particle is then established by comparing the track diameters with known stopping powers of various particles at that momentum. In this manner, areal densities can now be measured not only through the knock-on deuteron diagnostic but also through diagnostics involving other charged particles such as the knock-on protons.

The knock-on proton diagnostic is somewhat similar to the knock-on deuteron diagnostic. The number of these particles is once again proportional to the areal density of the layer in which they are produced (such as the plastic shell). The spectrum of these particles, however, is significantly different from the deuterons, and a different analysis must be used to interpret the measurement. In earlier work, a somewhat modeldependent technique to interpret the proton signal was presented. ${ }^{4}$ A more model-independent analysis of the proton spectrum can be devised that relies on the number of knock-on protons in a suitably defined energy range and is equally applicable to current experiments. Details of this analysis will be presented elsewhere. ${ }^{7}$

Here we present a novel knock-on deuteron-based diagnostic that will simultaneously diagnose three regions of a compressed target consisting of DT gas enclosed in a $\mathrm{CH}$ shell overcoated by $\mathrm{CD}$. This diagnostic is primarily based on measuring the knock-on deuteron spectrum and relies on knock-on protons for an independent measurement of the areal density of the plastic layers. Self-consistency would then dictate a favorable comparison between the values of the areal density of the $\mathrm{CH}$ layer inferred using the deuterons and protons.

In a direct-drive ICF target implosion, degradation in target performance is believed to occur primarily through RayleighTaylor instability, ${ }^{8}$ which is seeded by either target imperfections or laser nonuniformity. This instability, occurring at the ablation surface, can then feed through to the rear surface of the shell (or the fuel-pusher interface) during the acceleration phase of the instability. During the deceleration phase, these distortions at the fuel-pusher interface can grow, resulting in a mixing of the fuel and the pusher. The knock-on particle spec- 
trum carries information about conditions in the target during this latter phase of the implosion. This is when core temperatures and densities are high enough to initiate the fusion process and to produce the knock-on ions. The mixing of the fuel and the shell at these times in the implosion can significantly modify the neutron production rate relative to onedimensional (1-D) simulations and consequently the production of the knock-on ions and their spectra. Current diagnostics on the mixing of various layers in the target use $x$-ray spectroscopic signatures from various dopants in the target. ${ }^{9}$ Techniques based on nuclear particles would provide an independent assessment of hydrodynamic mix in the target and could probe conditions in the target that are not easily accessible by $\mathrm{x}$-ray spectroscopy. In addition, the knock-on deuteron diagnostic when used in conjunction with other diagnostic tools such as the neutron temporal diagnostic (NTD) ${ }^{10}$ (which can measure the thermonuclear burn history of a target in an experiment) may serve to probe conditions in the compressed target at the onset of significant mix due to hydrodynamic instabilities.

In the sections that follow, we discuss (1) the knock-on particles from a typical target consisting of only two regions: DT fuel and a plastic (CH) shell. Knock-on deuterons (and tritons) are produced in the fuel, and knock-on protons are produced in the $\mathrm{CH}$ shell. (2) We then discuss specifically the knock-on diagnostic in the context of the elastically scattered deuterons and protons. (3) A generalization of this technique infers $\rho R$ in three regions of the compressed target, using the detailed shape of the deuteron spectrum. The target involved has three layers: DT, CH and, CD. Knock-on deuterons are produced in both the DT and CD layers with two well-defined high-energy peaks in the spectrum, separated by an energy determined by the areal density of the intermediate plastic layer. Using the result from simulation as an example, we demonstrate the procedure for deducing the $\rho R$ of the three layers from this spectrum. (4) We discuss how hydrodynamic instabilities could modify the measured knock-on deuteron spectrum and examine the validity of our analysis for these modified spectra. Finally, we mention briefly how we might compare our inferred results from experimental measurements to simulation.

\section{Knock-on Ions as a Diagnostic for $\rho \boldsymbol{R}$}

The knock-on diagnostic relies on the elastic scattering of various ions (deuterons and protons) in the target from the 14.1-MeV primary DT neutrons (Fig. 77.17). The number of such elastically scattered particles, $N_{K}$, is then proportional to the number of primary neutrons, $Y$, the number density for the particle of interest, $n_{K}$, the average distance that the neutron traverses in the target, $\langle R\rangle$, and is given by

$$
N_{K}=n_{K}\langle R\rangle \sigma_{K} Y
$$

where the subscript $K$ indicates the type of knock-on particle and $\sigma_{K}$ is the corresponding cross section for elastic scattering ( $0.64 \mathrm{~b}$ for deuterons and $0.69 \mathrm{~b}$ for protons). Using the relation between the ion number density and the mass density, this can be rewritten for the number of knock-on deuterons produced in equimolar DT as

$$
N_{D}=7.7 \times 10^{-2} Y\langle\rho R\rangle \mathrm{cm}^{2} / \mathrm{gm},
$$

where $\langle\rho R\rangle$, the areal density, is given by

$$
\langle\rho R\rangle \equiv \int_{0}^{R} \rho d r
$$

The ratio of the number of knock-on deuterons to the number of 14.1-MeV DT primary neutrons provides a measure of the fuel's areal density. We note that knock-on tritons can also be produced in a similar elastic-scattering process with the energetic DT neutrons.

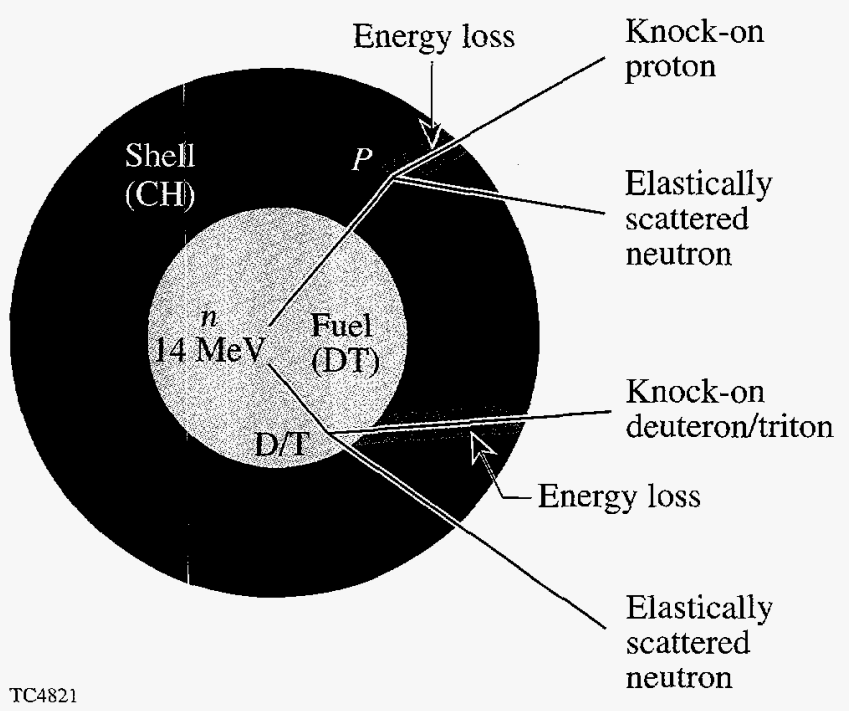

Figure 77.17

Knock-on process. 
Knock-on protons may be produced by the addition of hydrogen to the fuel or from the protons in the plastic, if the target is prepared with a plastic shell. For knock-on protons produced from the plastic $(\mathrm{CH})$ layer, Eq. (1) for the number of elastically scattered protons can be rewritten as

$$
N_{P}=3.2 \times 10^{-2} Y\langle\rho R\rangle \mathrm{cm}^{2} / \mathrm{gm} .
$$

Again, the ratio of the number of elastically scattered protons to the number of DT neutrons is proportional to the areal density of the plastic layer.

In an experiment, however, it is not possible to detect all the knock-on particles produced. The CPS has a finite solid angle and therefore samples only a fraction of the knock-ons produced. Therefore, an assumption about isotropy in knockon production is required to infer the total number of knockon particles produced. (Deviations from isotropy can be checked because there are two CPS's that view the target from different directions).

The more challenging aspect of inferring the number of knock-ons produced relates to the spectrum of these particles. The knock-on spectrum is produced over a continuum of energies extending over several $\mathrm{MeV}$ (knock-on deuterons occur up to $12.5 \mathrm{MeV}$, while the proton spectrum extends up to 14.1 MeV) due to different neutron-impact parameters. The entire spectrum cannot be measured because the very lowenergy knock-ons can be stopped in the target or in the filter in front of the CPS. Also, the very high density of background tracks at lower energies makes the measurement of the entire spectrum challenging. As a result, these diagnostics rely on the identification of specific features of the knock-on spectra to deduce the total number of ions produced and hence the areal density of the layer of interest.

\section{Knock-on Deuterons}

Even though the entire knock-on deuteron spectrum cannot be measured, the number of knock-ons produced can be reliably deduced using the high-energy region of the spectrum. The anisotropic differential cross section for elastic scattering results in a clearly identifiable peak in the spectrum (shown schematically in Fig. 77.18). The number of deuterons under this peak is about $16 \%$ of the total number of deuterons produced in the scattering process and is relatively independent of any distortion of the spectrum that may occur due to the slowing down of the deuterons in the target for a large range of areal densities. This useful feature allows a model-indepen-

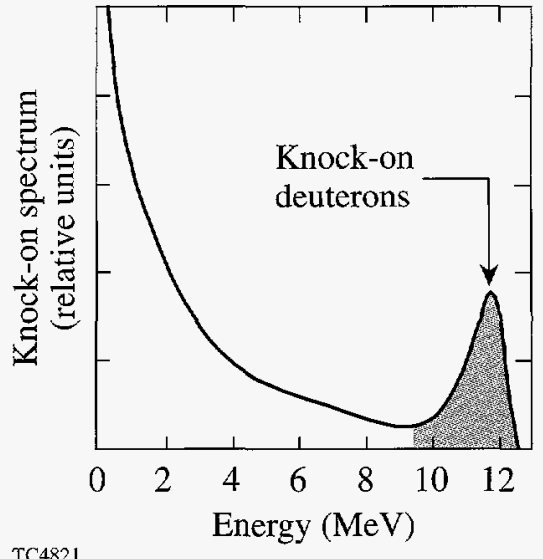

Figure 77.18

Schematic spectra of the knock-on particles.

dent inference of the total number of knock-on deuterons produced [and hence the $\rho R$ of the fuel, $(\rho R)_{f}$, through Eq. (2)].

In addition to the fuel areal density, the knock-on deuteron spectrum can also provide a measure of the shell's $\rho R$. Knockons produced in the target slow down (primarily through energy loss in the shell), and as a result the spectrum is downshifted from its usual maximum of $12.5 \mathrm{MeV}$. Figure 77.19(a) shows the spectra due to different areal densities of the shell, $(\rho R)_{\mathrm{CH}}$, calculated using a straight-line transport of the knock-on deuterons. The continuous energy loss of these charged ions is modeled using Ref. 11. The slowing down of the deuterons can be characterized by the end point of the spectrum (defined as the higher of the two energies of the halfmaximum of the peak). As Fig. 77.19(b) indicates, this end point is proportional to the areal density of the shell, and this feature can be used to deduce this quantity in experiment.

An important feature of the knock-on deuteron diagnostic that enables a relatively model-independent measure of the shell's $\rho R$ is the temperature insensitivity of the high-energy peak of the deuteron spectrum. Figure 77.20 shows the deuteron spectra for two different shell $\rho R$ values at two different typical electron temperatures (the temperatures are typical of the shell in 1-D simulations of the implosions). Energy loss at these typical densities and temperatures in imploding ICF targets is dominated by losses to electrons (the electron density is related to the ion density and consequently the areal density of the material through its degree of ionization). Knock-on deuterons with energies greater than about $7 \mathrm{MeV}$ typically have much higher velocities than electrons at the typical 

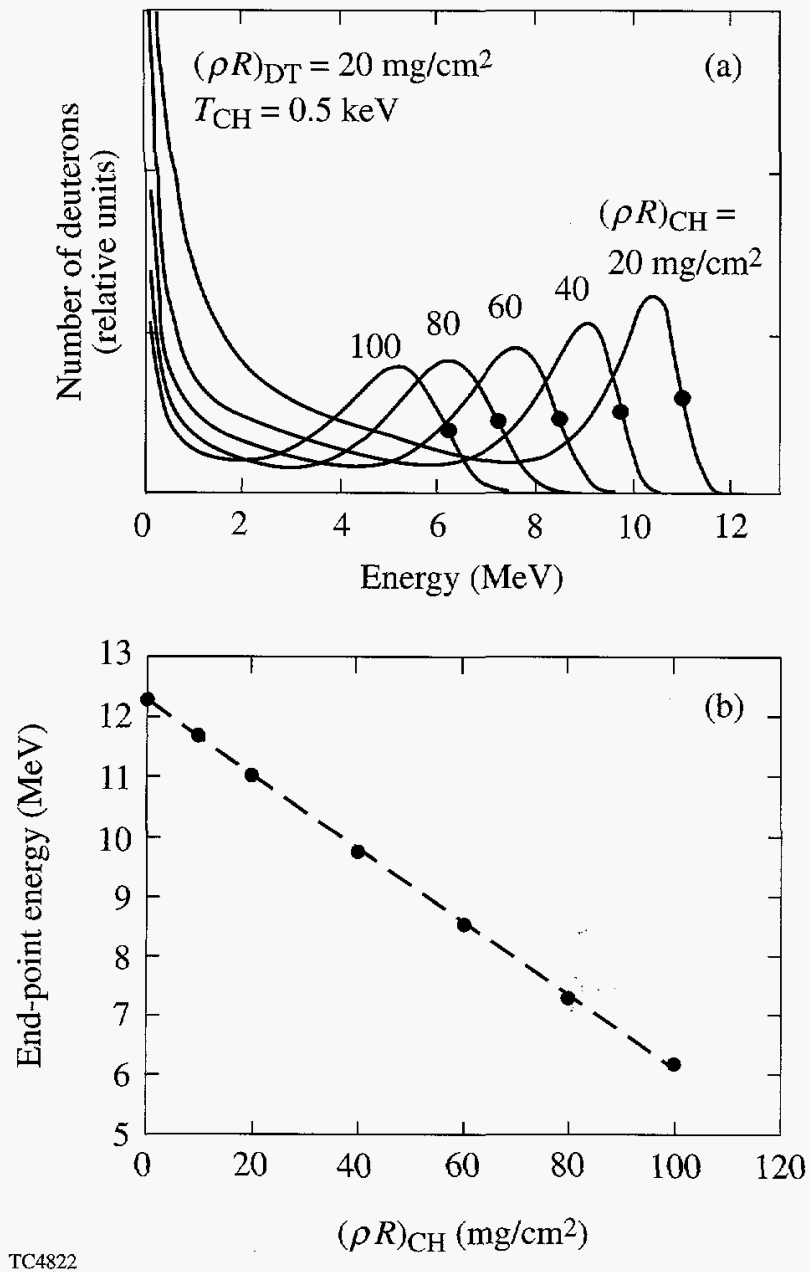

Figure 77.19

(a) The slowing down of the knock-on deuteron spectrum for different areal densities of the shell, $(\rho R)_{\mathrm{CH}}$. (b) End-point energy of the spectrum as a function of the shell areal density.

temperatures in the cold plastic $(\sim 0.5 \mathrm{keV})$. In this limit, the energy loss is independent of the electron's temperature and is thus dependent only on the shell's $\rho R$. As Fig. 77.19(a) indicates, for $\rho R \geqslant 60 \mathrm{mg} / \mathrm{cm}^{2}$, the deuterons are slowed to less than $7 \mathrm{MeV}$. This value of $\rho R$ suggests a limit on the maximum value of the shell's areal density that can be deduced independent of temperature considerations in the shell. On the other hand, the knock-on tritons, being more massive, show a greater sensitivity to both the temperature and the $\rho R$ of the shell, limiting the range of temperatures and areal densities over which conditions in the target can be inferred reliably from their spectrum. However, the triton spectrum can be used as a self-consistency check on target conditions that have been measured by other diagnostics.

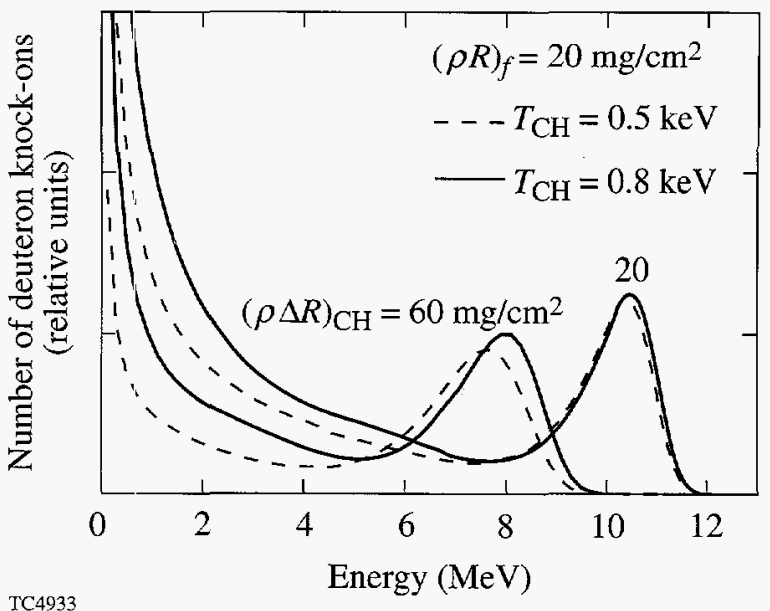

Figure 77.20

The deuteron spectrum is relatively insensitive to temperatures in the cold portions of the target.

Detailed knock-on spectra have been recently measured experimentally using the CPS. These preliminary spectra show good agreement with those obtained from simulations of implosions. In the next section, we consider a generalization of the knock-on deuteron diagnostic that will enable more detailed information about the compressed target.

\section{"Three-Layer" Targets}

This extension of the deuteron diagnostic uses a target comprising three layers (shown in Fig. 77.21): an inner DT (or a $\mathrm{DT}+{ }^{3} \mathrm{He}$ ) fuel region, a plastic $(\mathrm{CH})$ shell, and an ablator (CD). This target is different from those considered previously, where only two layers (DT and $\mathrm{CH}$ ) were present. Its characteristics and dimensions are commensurate with targets surrogate to future cryogenic targets designed for the OMEGA laser, ${ }^{12}$ and the diagnostic design permits some flexibility in each layer's thickness while retaining its equivalence to currently used surrogate targets.

Several energetic nuclear particles, shown schematically in Fig. 77.21, can be used to diagnose areal densities. Knock-on protons are produced in the plastic, and the areal density of the plastic layer can be deduced from the ratio of the number of protons produced to the number of primary neutrons. The addition of ${ }^{3} \mathrm{He}$ to the DT fuel is optional. The presence of ${ }^{3} \mathrm{He}$ in the target, however, provides another independent measure of areal density; the energy loss of the energetic 14.7-MeV proton from the $\mathrm{D}^{3}{ }^{3} \mathrm{He}$ reaction is proportional to the total areal density of the target. ${ }^{4}$ 


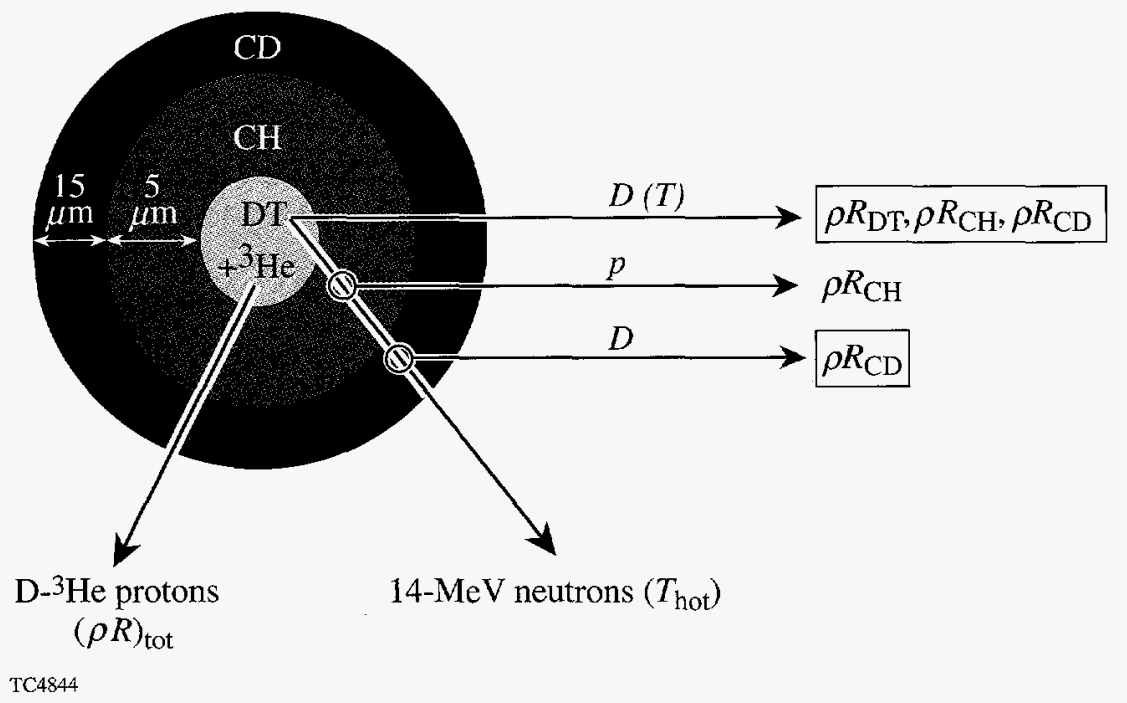

Figure 77.21

Sources of particles for the diagnosis of areal densities in three-layer targets.

Knock-on deuterons are produced in both the fuel and the $\mathrm{CD}$ layer. The deuterons produced in the fuel lose energy as they traverse the target, causing the high-energy peak to shift downward. The spectrum of deuterons from the CD layer, on the other hand, has its maximum energy at the nascent value of $12.5 \mathrm{MeV}$. The complete simulated spectrum for a target with a combined ablator and shell thickness of $20 \mu \mathrm{m}(5 \mu \mathrm{m} \mathrm{CH}$ and $15 \mu \mathrm{m} \mathrm{CD}$ ) is shown in Fig. 77.22. The target is driven with a 1-ns square pulse, which has been chosen arbitrarily. The spectrum is produced from a 1-D simulation of the implosion using the hydrodynamic code $L I L A C^{13}$ and a post-processor that transports the knock-ons in straight lines for every time

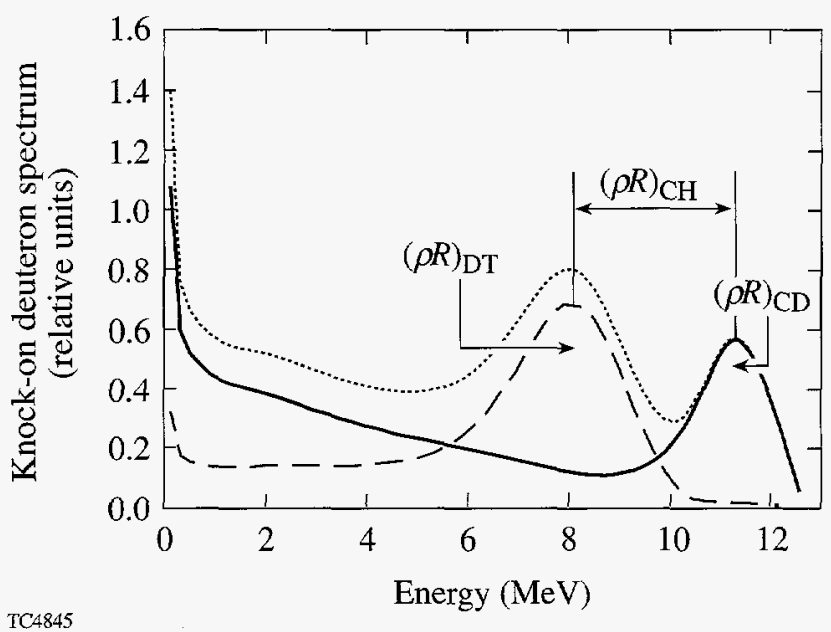

Figure 77.22

Simulated knock-on deuteron spectrum from a three-layer target with contributions from the individual layers. step using the simulated density and temperature profiles while accounting for their energy loss. The relevant features are the two high-energy peaks in the spectrum arising from the individual contributions of the fuel and $C D$ layers (shown as dashed lines in Fig. 77.22). The area under the higher-energy peak is primarily a measure of the areal density of the CD layer, $(\rho R)_{\mathrm{CD}}$, whereas the peak at the lower energy has contributions from both the fuel and the CD shell. The separation of the two peaks provides a measure of the areal density of the plastic layer, $(\rho R)_{\mathrm{CH}}$. We note that for the typical electron temperatures in the cold shell and ablator, the energy range spanned by the peaks of such a deuteron spectrum is fairly temperature insensitive. This temperature independence will be exploited later to deduce the areal densities of the three regions of the target from the data.

The areal densities of the three layers can be deduced nearly model independently using the scheme outlined previously, if the peaks are well separated. We first consider the areal density of the plastic layer. For the spectrum shown in Fig. 77.23(a) (a solid line) the separation of the two high-energy peaks is about $3 \mathrm{MeV}$. The areal density of the plastic resulting in this separation should correspond to that value that results in a downshift of the end-point energy by the same amount. From Fig. 77.19(b), this separation corresponds to an areal density of about $40 \mathrm{mg} / \mathrm{cm}^{2}$ to be compared with the value of $35 \mathrm{mg} / \mathrm{cm}^{2}$ in the simulation. Next, to deduce the areal density of the CD layer, we calculate the total number of deuterons in the highenergy peak. This value is a known fraction of the total number of deuterons produced since this portion of the spectrum is unaffected by the presence of deuterons from the fuel. For the 

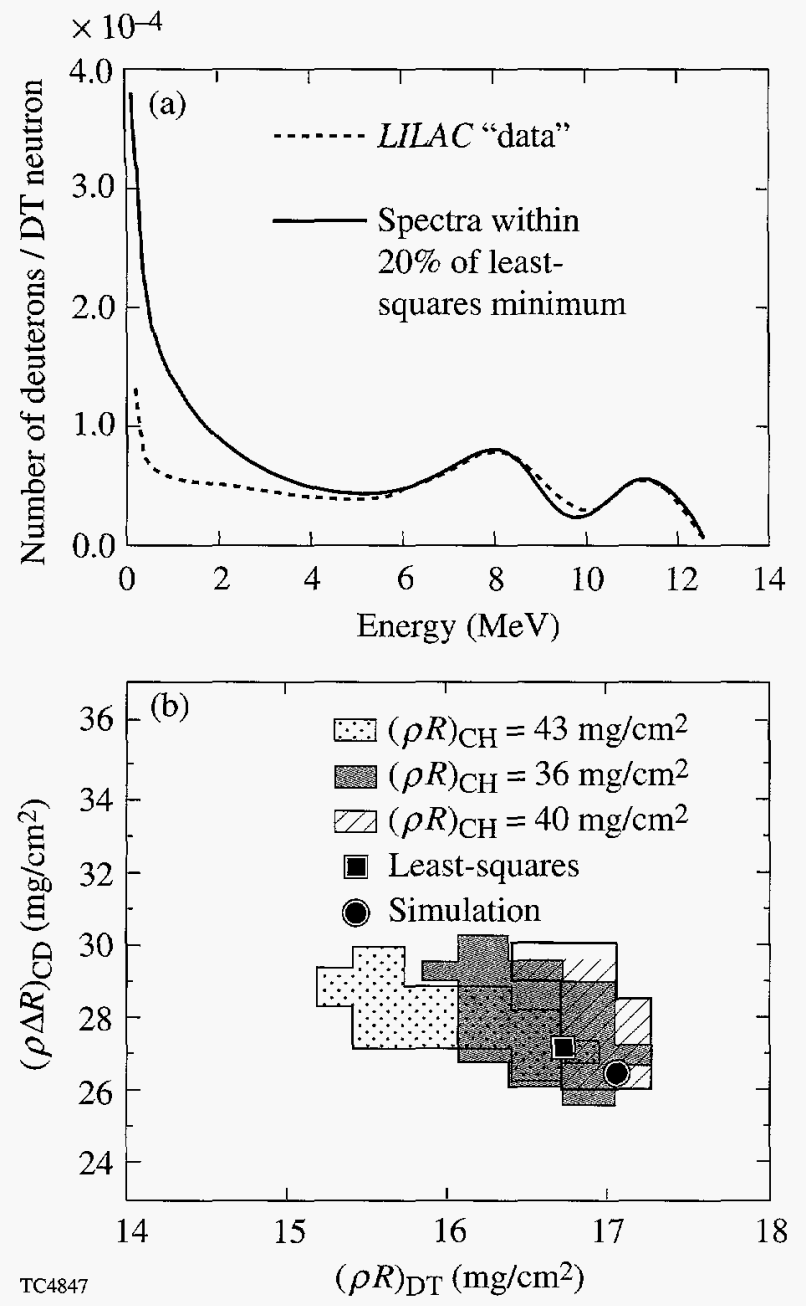

Figure 77.23

(a) Comparison of test (simulated) spectrum (solid line) showing spectrum from best-fit model (dashed line). (b) Areal densities for the three layers that result in spectra whose difference squared is within $20 \%$ of the least-squares value. Each shaded region represents the set of areal densities of the fuel and CD layer satisfying the $20 \%$ criterion, for a fixed value of the areal density of the $\mathrm{CH}$ layer.

spectrum (solid line) in Fig. 77.23(a), this is the number of deuterons above $10.25 \mathrm{MeV}$, and again, from the spectra in Fig. 77.19 (a) this corresponds to about $12 \%$ of the total knockon deuterons produced. Using this fraction for the number of deuterons in the peak and a formula for CD [similar to Eq. (2)], we obtain a value of $26.5 \mathrm{mg} / \mathrm{cm}^{2}$ for the $C D$ layer that compares favorably with the value of $25.6 \mathrm{mg} / \mathrm{cm}^{2}$ in the simulation. While the areal densities of the $\mathrm{CH}$ and $\mathrm{CD}$ layers can be determined model independently, some uncertainty is introduced in the value of the areal density of the fuel, $(\rho R)_{f}$, since not all the deuterons under the low-energy peak are produced in the fuel. The contribution to this peak from the $\mathrm{CD}$ layer depends on the slowing down of the deuterons and the geometry of the paths through the target. An upper limit for $(\rho R)_{f}$ can be obtained by assuming that all the deuterons under this peak are produced in the fuel. In this case, the inferred areal density of the fuel using Eq. (2) is $23.6 \mathrm{mg} / \mathrm{cm}^{2}$ to be compared with the simulation value of $17.0 \mathrm{mg} / \mathrm{cm}^{2}$.

This uncertainty in the value of the inferred fuel areal density can be mitigated through a different analysis of the knock-on deuteron spectrum. We consider deuteron spectra from a model where each layer is approximated by a constant density and temperature (an ice-block model). The density and the thickness of each layer are chosen by requiring a fixed mass for each layer (known from the specifications of the target being modeled) and a chosen $\rho R$. We note once again that since the deuteron spectrum is insensitive for the ranges of areal densities expected in such implosions, temperatures in the colder plastic and CD can be ignored in this analysis. The choice of fuel temperature, however, cannot be made arbitrarily since the deuterons may lose some energy in the hot fuel. In this example, we choose the fuel temperature at peak neutron rate in the simulation as the relevant fuel temperature. In deducing areal densities from the experimentally measured spectrum, the temperature obtained experimentally from the width of the DT neutron spectrum ${ }^{14}$ (measured through timeof-flight techniques) should be used in the model.

In this manner we construct a static representation of the target and fit the spectra from such a model by varying the areal densities of each layer. While the ice-block model is not expected to accurately describe the primary complexities of an imploding target such as the spatial and time-dependent variation of densities and temperatures, the spatial localization of neutron sources in the target, and the geometry of the knockon trajectories through the target, it should provide a reasonable time and spatially averaged representation of the target relevant to the knock-on spectrum.

To test our scheme for deducing the areal densities, we consider again the simulated spectrum shown as a solid line in Fig. 77.23(a). Using the model described above, we vary the $\rho R$ of the three layers to minimize the least-squares difference between the spectrum from the model and the data. The energy range chosen for this minimization is the area determining the two peaks in the spectrum ( $\approx 5 \mathrm{MeV}$ ). The technique for minimization we choose is based on the Downhill Simplex Method of Nelder and Mead. ${ }^{15}$ If we assume that the neutrons are created uniformly in the fuel, the resulting spectrum of 
such a minimization scheme is shown as the dashed line in Fig. 77.23(a). Our values for the areal densities for the DT, CH, and $C D$ layers $\left(16.5,40\right.$, and $27.4 \mathrm{mg} / \mathrm{cm}^{2}$, respectively) compare favorably with the results from the simulation $(17,35$, and $25.6 \mathrm{mg} / \mathrm{cm}^{2}$ ). These values agree very well with the model-independent extraction of the areal densities of the $\mathrm{CH}$ and CD layers, implying correctly well-separated peaks and, in addition, provide a tighter bound on $(\rho R)_{f}$.

To gauge the sensitivity of the spectrum to the least-squares values of $\rho R$ obtained in this manner, we consider Fig. 77.23(b), which shows sets of $(\rho R)_{\mathrm{CD}}$ and $(\rho R)_{f}$ for different values of $(\rho R)_{\mathrm{CH}}$. [Each shaded region represents a set of areal densities of $\mathrm{CD}$ and fuel, corresponding to a certain value of $(\rho R)_{\mathrm{CH}}$.] For each value of $(\rho R)_{\mathrm{CH}}$, this set corresponds to those values whose spectra are within $20 \%$ of the least-squares value. This range of areal densities of CD and the fuel has been obtained by fixing the areal density of the plastic in the model to the required value and varying the areal densities of the $C D$ and fuel layers. In this manner, we find the range of acceptable values of the areal densities of each layer in the target. Also shown in the figure is the least-squares value (square) and the result from the $1-\mathrm{D}$ simulation (circle). We see that by using this procedure we obtain values of $\rho R$ of the fuel and CD layer to within $10 \%$ of the true value. The larger range of acceptable values of $(\rho R)_{\mathrm{CH}}$ (35.5 to $43 \mathrm{mg} / \mathrm{cm}^{2}$ for the plastic layer compared to 15 to $17.2 \mathrm{mg} / \mathrm{cm}^{2}$ for the fuel and 25.5 to $29.1 \mathrm{mg} / \mathrm{cm}^{2}$ for the CD layer) indicates that the deuteron spectrum is less sensitive to the areal density of the plastic layer. This is probably due to the fact that $(\rho R)_{\mathrm{CH}}$ does not determine an absolute number or energy; the relative separation of the two high-energy peaks is determined by this value. A comparison with the value deduced from the knock-on proton spectrum would, in addition, provide an independent check on the value of $(\rho R)_{\mathrm{CH}}$. Finally, we note that the true set of areal density values obtained from the simulation is not excluded from our result at this $20 \%$ level, thus providing a measure of the sensitivity of the spectrum to the three areal densities.

If we assume that the neutrons are produced in the center of the fuel and repeat the above analysis, we obtain the following results: an areal density of $16.3,40.4$, and $30.5 \mathrm{mg} / \mathrm{cm}^{2}$ for DT, $\mathrm{CH}$, and $\mathrm{CD}$, respectively. The least-squares difference between the model spectra and the test data for this case is higher than for the uniform source $\left(4.3 \times 10^{4}\right.$ and $2.1 \times 10^{4}$, respectively), implying correctly a uniform distribution of the DT neutrons in the simulation.

\section{Modification of the Knock-on Deuteron Spectrum due to Mix}

Our discussion has so far been based on a 1-D simulation of the implosion that does not include the effects of hydrodynamic instabilities and mix on the imploding target. In addition, any effects on the target due to long-wavelength asymmetries (possibly due to laser-beam imbalances in power and pointing errors) have also been ignored. The effects of such departures on nuclear and particle diagnostics are difficult to determine quantitatively from 1-D simulation.

During the deceleration phase, the Rayleigh-Taylor unstable fuel-pusher interface, seeded by its nonuniformity, can result in a mixing of the hot fuel and cold pusher. This mixing of materials at very different temperatures can result in a significant quenching of the neutron yield relative to $1-D$ simulations (that do not include this effect). Since the diagnostic should probe conditions in the compressed target corresponding to times of peak neutron and consequently knock-on production, this quenching can result in different conditions probed experimentally by the diagnostic relative to 1-D simulations. For the purposes of studying the feasibility of the diagnostic in the presence of such mixing, we assume that the effect of the deviations from 1-D is to exclusively change the neutron-production rate and hence the knock-on spectrum. In other words, the effect of such departures from 1-D behavior on the implosion dynamics is ignored.

To assess the effect of this mixing, we compare the spectra from purely 1-D simulations with two models of neutron rate truncation. These models should span the extremes of possible neutron rate truncations in the experiment. In the first model, we assume that a portion of the fuel implodes with a constant velocity acquired just before deceleration begins and is unaffected by the growing instabilities at the fuel-pusher interface. We then assume that the only neutron yield is from this portion of the fuel. The neutron rate from this model is shown in Fig. 77.24(a) as the free-fall rate and is significantly lower in magnitude relative to the $1-\mathrm{D}$ simulation. Figure 77.24 (b) shows the corresponding areal densities in the target from the simulation. As Figs. 77.24(a) and 77.24(b) indicate, the neutron rate in this model peaks earlier and thus probes earlier times in the implosion. This results in a deuteron spectrum (dotted line in Fig. 77.25) that is characteristic of smaller areal densities for all three layers. Our analysis provides values that agree reasonably with the results from simulation; the leastsquares values are $9.8,22.5$, and $17.1 \mathrm{mg} / \mathrm{cm}^{2}$, whereas the results of the simulation are $8.9,26.7$, and $15.8 \mathrm{mg} / \mathrm{cm}^{2}$. We once again note that the independent measurement of $(\rho R)_{\mathrm{CH}}$ 

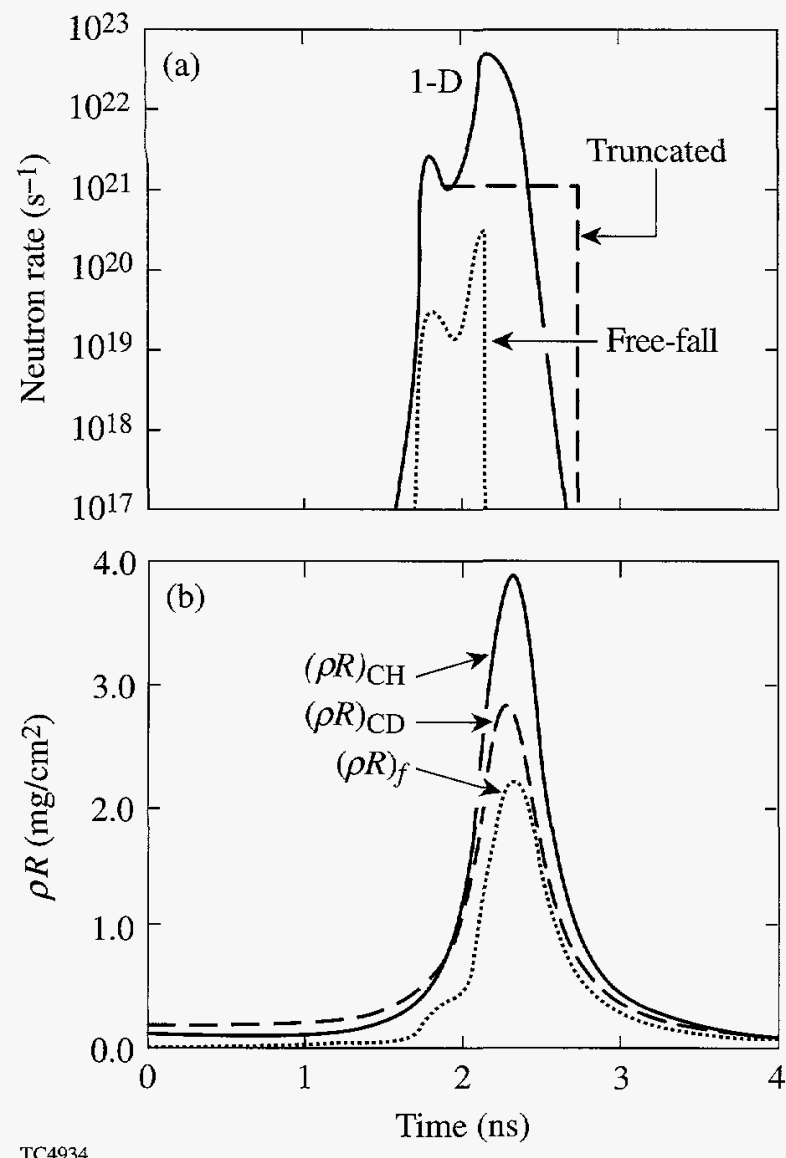

Figure 77.24

(a) Neutron rate curves for different models used for assessing the modification of the deuteron spectrum due to hydrodynamic mix: solid line-result of a 1-D simulation; dotted line-neutron rate obtained from a free-fall model (see text); and dashed line - neutron rate fixed to a constant value at a certain time. (b) $\rho R$ history of the target from 1-D simulations. In the simulations, the diagnostic is sensitive to areal densities near peak neutron rates and consequently peak compression.

using the knock-on proton spectrum can constrain the areal density of plastic inferred from the deuteron spectrum. The favorable comparison between the values of the areal densities inferred from the diagnostic and the true values suggests that the areal densities can still be deduced reasonably were such a modified spectrum the result of a measurement.

In a different model, we assume that the neutron rate proceeds as given by the 1-D simulation up to a certain time, and, thereafter, it proceeds at a constant rate given by the rate at the chosen time. This is shown in Fig. 77.24(a) as the constant burn rate model. A comparison with Fig. 77.24(b) indicates that the diagnostic then probes the times corresponding to the steep

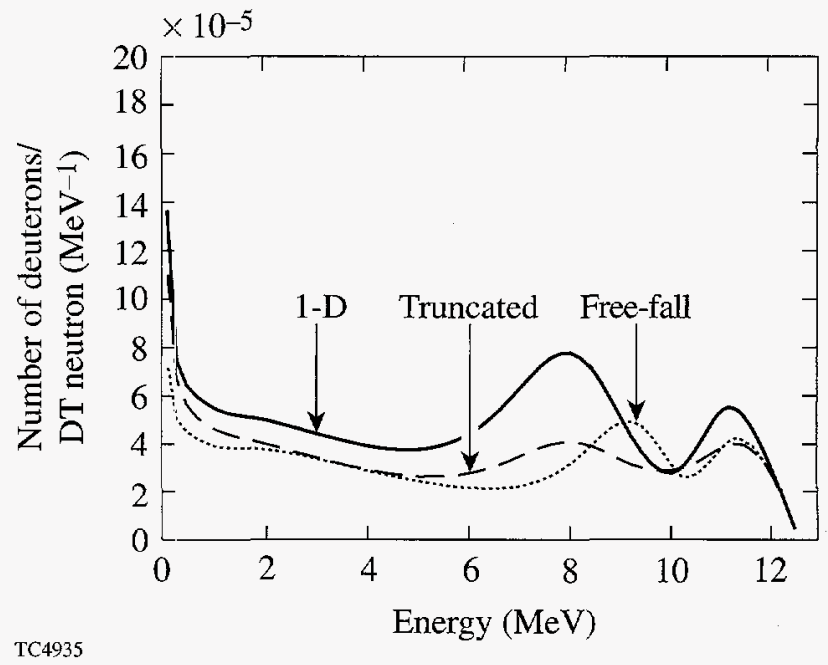

Figure 77.25

Knock-on deuteron spectra using the three models of neutron rate truncation shown in Fig. 77.24(a).

changes in the areal density. The significant neutron rate for a large fraction of time over which these changes in areal density occur in the target results in a considerably broadened emergent spectrum with less well defined peaks (dashed line in Fig. 77.25), which are to be compared with the results of the simulation $\left(10.7,32.3\right.$, and $\left.16.0 \mathrm{mg} / \mathrm{cm}^{2}\right)$. Nevertheless, the least-squared values $\left(8.9,22.9\right.$, and $\left.18.7 \mathrm{mg} / \mathrm{cm}^{2}\right)$ compare favorably with the results of the simulation $(17.9,36.0$, and $23.0 \mathrm{mg} / \mathrm{cm}^{2}$ ), suggesting that our analysis can be used to reliably infer the areal density of each of the layers, even when the peaks in the spectrum are less well defined.

Experimentally, the neutron rate history can be obtained through the neutron temporal diagnostic (NTD). ${ }^{10}$ One method to compare the implosion with 1-D simulations could be as follows: The experimentally obtained neutron rate curve could be used to identify the times in the implosion probed by the diagnostic - the diagnostic probes times around the peak neutron burn rate. An identification of these times would allow us to calculate the areal densities of the three layers from the simulation. A comparison of these values with those obtained from the knock-on diagnostic would shed light on whether conditions in the experiment compare favorably with the 1-D simulation up to the time probed by the diagnostic. If the areal densities inferred from the diagnostic differ considerably from those in the simulation, this procedure will allow one to identify a time, when mixing effects have already significantly influenced the fusion processes. Independent of any comparison with detailed hydrodynamics simulations, the areal densi- 
ties deduced from the knock-on deuteron diagnostic should be nearly model independent and would provide information about the conditions in the target corresponding to times in the implosion identified using the NTD. Further, a comparison with detailed mixing models may enable the identification of conditions in the target that would result in the observed neutron rate curves and the inferred values of $(\rho R)$.

\section{Summary and Conclusions}

In this article, we have presented a new diagnostic based on knock-on deuterons, which will simultaneously diagnose the areal densities in three different regions of the compressed ICF target. These targets have three layers (DT, $\mathrm{CH}$, and CD), and the areal density of each of these layers can be inferred from the deuteron diagnostic. In addition, knock-on protons from the $\mathrm{CH}$ layer can be used to independently deduce the areal density of the plastic.

When used in conjunction with a detector that measures the neutron rate history of an implosion (NTD), the time in the implosion probed by this diagnostic can be identified. This will permit a more detailed comparison between the simulation and experiment.

We have also examined the modification of the knock-on deuteron spectrum due to departures from 1-D behavior such as mixing. We conclude that while the spectrum may be influenced significantly by such departures from 1-D behavior, our method for analyzing the experimental spectrum should still reliably infer the areal densities in the three layers. Detailed mixing models would be required, however, to make any inferences about the mixing process in implosions. Experiments to measure these spectra from an imploding target are currently underway, and the results will be presented elsewhere.

\section{ACKNOWLEDGMENT}

This work was supported by the U.S. Department of Energy Office of Inertial Confinement Fusion under Cooperative Agreement No. DE-FC0392SF19460, the University of Rochester, and the New York State Energy Research and Development Authority. The support of DOE does not constitute an endorsement by DOE of the views expressed in this article.

\section{REFERENCES}

1. G. S. Fraley et al., Phys. Fluids 17, 474 (1974).

2. S. Skupsky and S. Kacenjar, J. Appl. Phys. 52, 2608 (1981).

3. M. D. Cable and S. P. Hatchett, J. Appl. Phys. 62, 2233 (1987).

4. Laboratory for Laser Energetics LLE Review 73, 15, NTIS document No. DOE/SF/19460-212 (1997). Copies may be obtained from the National Technical Information Service, Springfield, VA 22161.

5. H. Nakaishi et al., Appl. Phys. Lett. 54, 1308 (1989).

6. D. G. Hicks, C. K. Li, R. D. Petrasso, F. H. Seguin, B. E. Burke, J. P. Knauer, S. Cremer, R. L. Kremens, M. D. Cable, and T. W. Phillips, Rev. Sci. Instrum. 68, 589 (1997).

7. P. B. Radha and S. Skupsky, "A Novel Charged-Particle Diagnostic for $\rho R$ in Compressed ICF Targets," in preparation.

8. Lord Rayleigh, Proc. London Math Soc. XIV, 170 (1883); G. Taylor, Proc. R. Soc. London Ser. A 201, 192 (1950).

9. D. K. Bradley, J. A. Delettrez, R. Epstein, R. P. J. Town, C. P. Verdon, B. Yaakobi, S. Regan, F. J. Marshall, T. R. Boehly, J. P. Knauer, D. D. Meyerhofer, V. A. Smalyuk, W. Seka, D. A. Haynes, Jr., M. Gunderson, G. Junkel, C. F. Hooper, Jr., P. M. Bell, T. J. Ognibene, and R. A. Lerche, Phys. Plasmas 5, 1870 (1998).

10. R. A. Lerche, D. W. Phillion, and G. L. Tietbohl, Rev. Sci. Instrum. 66, 933 (1995).

11. S. Skupsky, Phys. Rev. A 16, 727 (1977); J. D. Jackson, Classical Electrodynamics, 2nd ed. (Wiley, New York, 1975).

12. F. J. Marshall, B. Yaakobi, D. D. Meyerhofer, R. P. J. Town, J. A. Delettrez, V. Glebov, D. K. Bradley, J. P. Knauer, M. D. Cable, and T. J. Ognibene, Bull. Am. Phys. Soc. 43, 1784 (1998).

13. E. Goldman, Laboratory for Laser Energetics Report No. 16, University of Rochester (1973).

14. H. Brysk, Plasma Phys. 15, 611 (1973).

15. W. H. Press et al., Numerical Recipes in FORTRAN: The Art of Scientific Computing, 2nd ed. (Cambridge University Press, Cambridge, England, 1992). 


\section{Arresting UV-Laser Damage in Fused Silica}

Deciding when to replace spot-damage-afflicted fused-silica optics or, in the case of inaccessible, space-based lasers, predicting the useful service life of fused-silica optics before catastrophic, pulsed-laser-driven crack growth shatters a part has recently become simpler. By empirically deriving a rule for laser-driven crack growth in fused silica as a function of the number of constant-fluence laser pulses, Dahmani et al. ${ }^{1}$ provided laser systems designers and operators with guidance on the crack-growth kinetics as well as on the stress-related ramifications such a laser-driven crack entails. Specifically, a hoop stress in the immediate vicinity of a crack growing along the beam propagation direction was identified as strongly coupling to both the laser fluence and the crack. ${ }^{2}$ It prompted the question of whether or not breaking the hoop-stress symmetry by some external perturbation will accelerate or stymie crack growth or, alternatively, will have no effect at all.

In this article, we report not only on the finding that, depending on the magnitude of a perturbing external stress, crack propagation in fused silica may slow relative to stressfree conditions, but also the more unexpected finding that the applied external stress raises the damage-initiation fluence. In gathering this evidence, a conventional experimental arrangement was used.

Pulses from a Nd:glass oscillator/single-pass amplifier system were frequency tripled in a dual-crystal KDP cell to yield temporally stable, 500-ps pulses at a repetition rate of one pulse every $10 \mathrm{~s}$. Prior to frequency conversion, the IR pulse was sent through a nonmagnifying, vacuum spatial filter. UV pulses were focused by a $2-\mathrm{m}$-focal-length lens to an $\sim 600-\mu \mathrm{m}$ spot size at the sample entrance surface. For each pulse, a record of the fluence distribution in this spot was acquired by a charge-injection-device camera located in a sample-equivalent plane and digitized to 8-bit accuracy. Spatially integrated UV energy per pulse was also monitored on each exposure. The UV beam-incidence direction was chosen to be a few degrees $(<10)$ off-normal to the sample entrance face to prevent (1) any back-reflection of residual, unconverted IR from seeding the amplifier in the backward direction, and (2) setting up a 351-nm interference pattern between sample entrance and exit surfaces that would invalidate the calculated fluence distribution. Damage initiation anywhere between the two sample surfaces, i.e., along the pulse-propagation direction, was recorded by $110 \times$-magnification dark-field microscopy. After damage initiation, the crack length was measured microscopically by viewing the sample orthogonally to the laserpulse propagation direction.

Fused-silica samples (Corning 7940, UV Grade A), with length $L=64 \mathrm{~mm}$, width $w=13.6 \mathrm{~mm}$, and thickness $t=$ $4.6 \mathrm{~mm}$, were conventionally pitch polished to laser quality on the entrance and exit surfaces and to cosmetic quality around the edges.

Samples were mechanically stressed by first centering each between apertured aluminum plates separately attached to a load cell (Eaton, Model 3397-25, maximum load capacity: $25 \mathrm{lbs}$ ). A predetermined, constant, uniaxial, compressive load was applied in such a manner that the compressive-force direction nearly coincided with the laser-pulse propagation direction (Fig. 77.26). The laser pulse entered and exited the samples through the apertures in the aluminum plates. The need for having beam-passage apertures ipso facto brings about stress conditions that vary from point to point within the aperture, both in magnitude and in principal directions (compressive or tensile). At first, this may appear as complicating data interpretation; for the following reason, however, it does not.

Laser-damage thresholds (for pulse lengths greater than picoseconds) are always reported as average values derived from a statistical number of sample sites per tested specimen. In all nondeterministic, i.e., extrinsic-impurity-driven, laserdamage processes the occurrence of damage hinges on the statistical presence or absence of one or more absorbing impurities within a given irradiated area. This statistical distribution in defect volume density is now convoluted by a site-tosite-varying stress distribution. In an ideal experiment, a large enough number of tests on samples and sites with precisely 


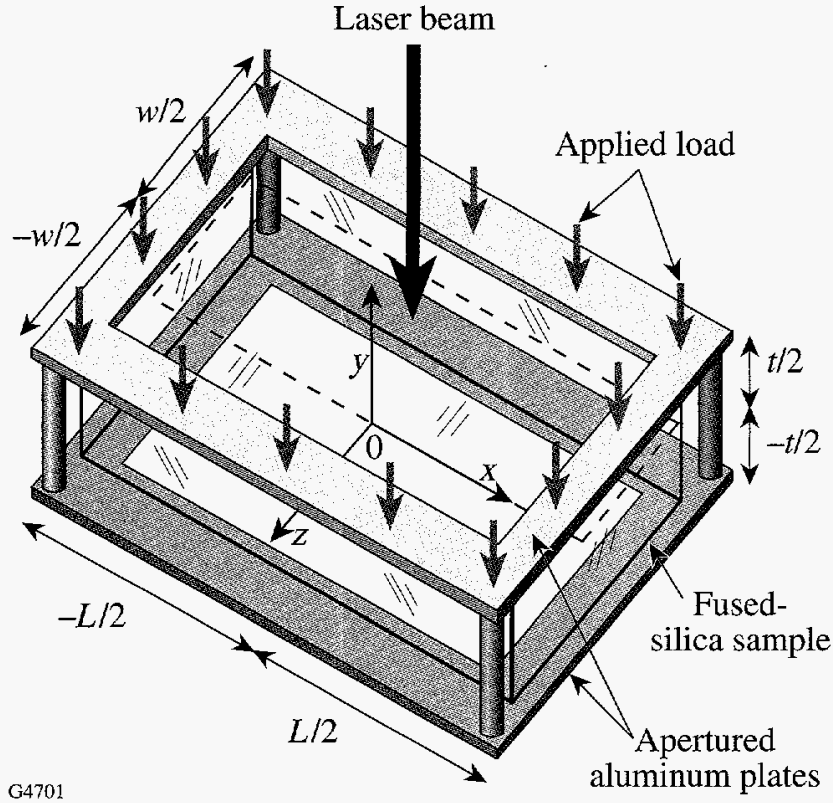

Figure 77.26

Experimental arrangement for applying compressive loads to fused-silica surfaces by clamping the conventionally polished sample between aluminum plates. The static pressure applied is measured by the load cell. Sample dimensions are length $L=64 \mathrm{~mm}$, width $w=13.6 \mathrm{~mm}$, and thickness $t=$ $4.6 \mathrm{~mm}$ oriented relative to the Cartesian coordinate system as indicated.

known local stress will deconvolve the two distributions; in practice, however, this is unrealistic. Rather, simulation of local stress conditions by finite-element methods, ${ }^{3}$ or when possible by analytic approaches, permits one to find with acceptable accuracy, for various aperture boundary conditions, the compressive and tensile stresses within the aperture, based on which one may choose many irradiation sites on a single sample. To guarantee good statistics, however, the current measurements still rely on more than one sample. All stress values quoted here are numerically derived from experimentally measured sample-loading conditions. The total applied force on the sample is $10 \mathrm{kgf}(1 \mathrm{kgf}=9.8 \mathrm{~N})$ in this case.

The onset of damage is defined as follows: for the given microscopic magnification and lighting conditions, any observable, permanent, irradiation-induced, surface or bulk modification. Throughout this article, initiation thresholds are reported for 1-on-1 mode, i.e., each sample site is irradiated only once. For well-known reasons, ${ }^{4}$ conventionally polished material of good bulk purity damages first at the exit surface. In these experiments, this is not only confirmed but damage propagation effects under multiple irradiation conditions are, for now, deliberately restricted to those events in which throughout damage-crack initiation and growth no entrance-surface damage is encountered, i.e., the laser fluence at the damage site remains unobscured by upstream obstacles.

We first report the effect of stress on damage initiation. In Fig. 77.27, the $351-\mathrm{nm}$, damage-onset fluence threshold is plotted for exit-surface damage $(\times$ symbols $)$ against applied stress magnitude (sign convention: + tensile, - compressive). Here the load is applied nearly collinear with the pulsepropagation direction. As is immediately evident, regardless of whether the stress character is tensile or compressive, threshold enhancements of up to $70 \%$ are attainable from modest stresses, and the largest, relative threshold-improvement increments can be garnered from the smallest stresses. Note how the symmetry around zero stress tends to imply that the underlying damage-initiation process is independent of whether the stress is compressive or tensile. Over how large a tensile-stress range this holds true is yet to be ascertained.

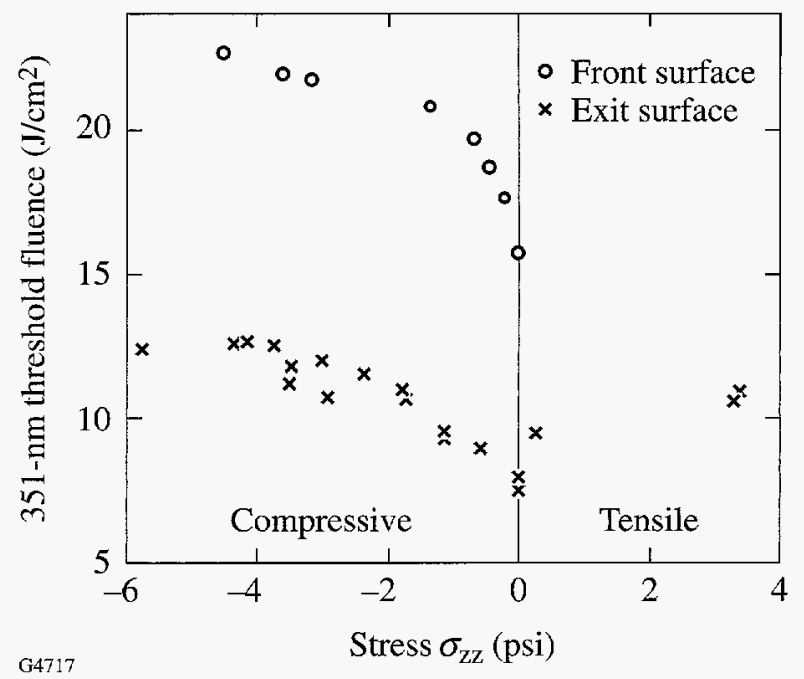

Figure 77.27

Entrance (x)- and exit $(o)$-surface, 351-nm damage-initiation threshold as a function of applied stress follows similar trends.

For the laser systems designer it is important to know if the beneficial stress effect is an exit-surface phenomenon only or if an equivalent advantage can be gained for the entrance surface as well. Collecting the data for which front-surface damage was incurred, i.e., data excluded so far, Fig. 77.27 also plots a similar trend for the entrance-surface damage-initiation threshold (open circles) as for the exit surface.

We next concentrate on laser-driven crack growth. Once the exit-surface damage-initiation threshold $F_{\text {exitthr }}$ is determined as described above, a flaw is deliberately created at a 
new site, which upon further irradiation becomes the source for both crack formation and crack propagation. Cracks observed in these experiments are not empty voids but are filled with granular glass debris that scatters light efficiently. The growth dynamics of such cracks as a function of incident fluence and number of exposures has already been reported elsewhere. ${ }^{2}$ To underscore the influence of stress on the crackgrowth kinetics, we compare here results obtained under extreme conditions, i.e., for irradiation of a flaw by 270 consecutive laser pulses of constant fluence $F_{L}=2.1 \times F_{\text {exit/thr }}$, a far-from-normal condition for most lasers. By choosing extreme irradiation conditions, crack-growth arrest is most convincingly demonstrated. Figure 77.28 displays side-byside micrographs of cracks formed in (a) the unstressed sample $\left(\sigma_{\mathrm{zz}}=0\right)$ and $(\mathrm{b})$ the stressed sample $\left(\sigma_{\mathrm{zz}}=-6 \mathrm{psi}\right)$. Note that in Fig. 77.28(a) the crack growth has pushed the crack tip beyond the field of view. A less-striking, though quantitative, account of crack arrest as a function of applied stress is displayed in Fig. 77.29, where the length of cracks from multiple sites, all irradiated at the above-fluence condition, is plotted against the stress prevailing at any particular site. The functional dependence on applied stress displayed in Fig. 77.29 offers promise: much can be achieved in altering, by modest stress, the crack-propagation outcome, while the empirical crack-length-reduction limit for larger stresses renders these unnecessary in practice. The concomitant penalty in stress-induced birefringence that such crack-growth preven- tion entails is also kept within bounds: the maximum stress of 6 psi plotted in Figs. 77.27 and 77.29 causes 0.5 -nm retardance. A second, intriguing ramification of the slope in Fig. 77.29 pertains to damage testing in general: there exists evidence 5 that the polishing process leaves a thin, densified layer of silica at/near the air interface, the stress within which may locally vary or may vary from sample to sample. Depending on the extent of such variation, the statistical error on measured surface-damage initiation thresholds should correspondingly be large since the slope in Fig. 77.27 is steepest near zero stress. An unfortunate paucity of reported 351-nm, fused-silica, surface-damage thresholds makes it, at this time, difficult to corroborate this correlation from literature data. In the same vein, there should be a damage-initiation stress effect for interfacial darnage on coated or cladded silica surfaces whenever the therraal-expansion mismatch between the substrate and the film stack or cladding material introduces interfacial stress. This would be most readily observable in antireflective coatings as these permit significant laser intensity to reach the substrate interface. Finally, there remains an urgent question to be resolved as to whether this phenomenon is unique to fused silica or may be present also in other glasses.

We presented here results from stress-inhibited laser-driven crack propagation and stress-delayed damage-initiation experiments in fused silica at $351 \mathrm{~nm}$. Within the stress interval of $-6 \leq \sigma_{\mathrm{zZ}} \leq 4 \mathrm{psi}$, the damage initiation threshold is raised by
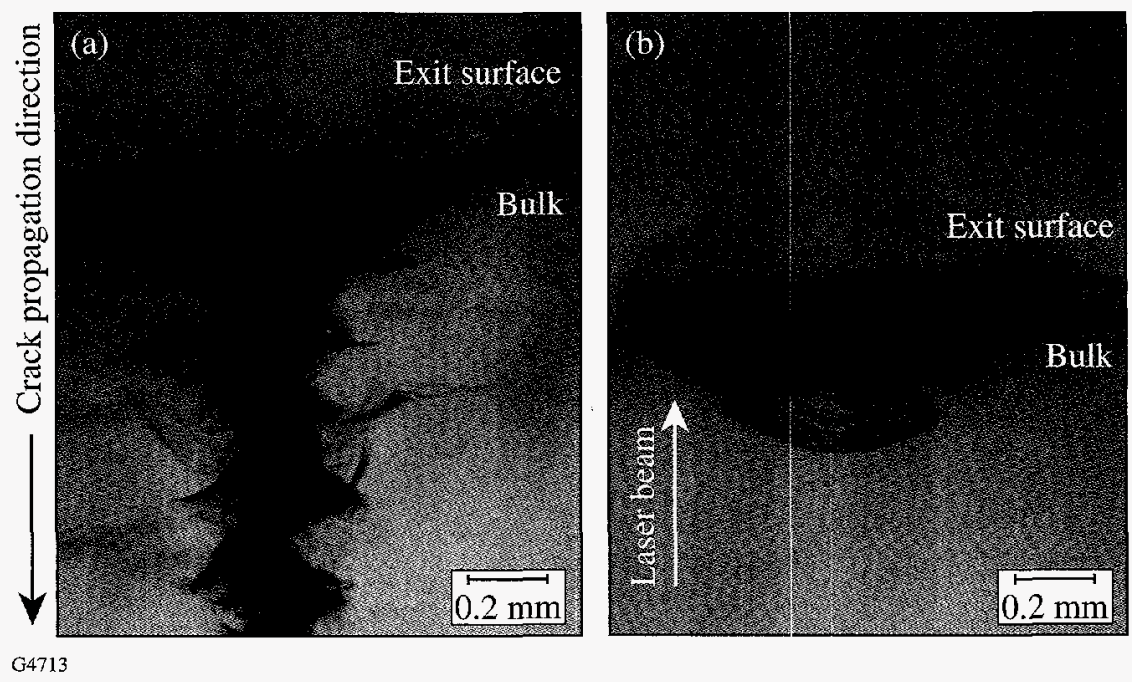

Figure 77.28

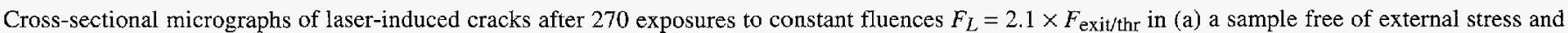
(b) a sample with $\sigma_{\mathrm{zz}}=-6$ psi. The crack tip in micrograph (a) is, for the given magnification, located already outside the field of view. 
$70 \%$. For such modest stresses, both compressive or tensile stresses appear to raise this threshold while keeping the induced-birefringence penalty $\leq 0.5-\mathrm{nm}$ retardation. The ramifications of these findings for large-aperture systems, such as OMEGA, are yet to fully emerge: aperture scaling must commence and the stress-magnitude regime must be extended to higher stresses in order to evaluate whether or not the apparent saturation (near-zero slope) at 6 psi remains. These tasks are in progress at this time.

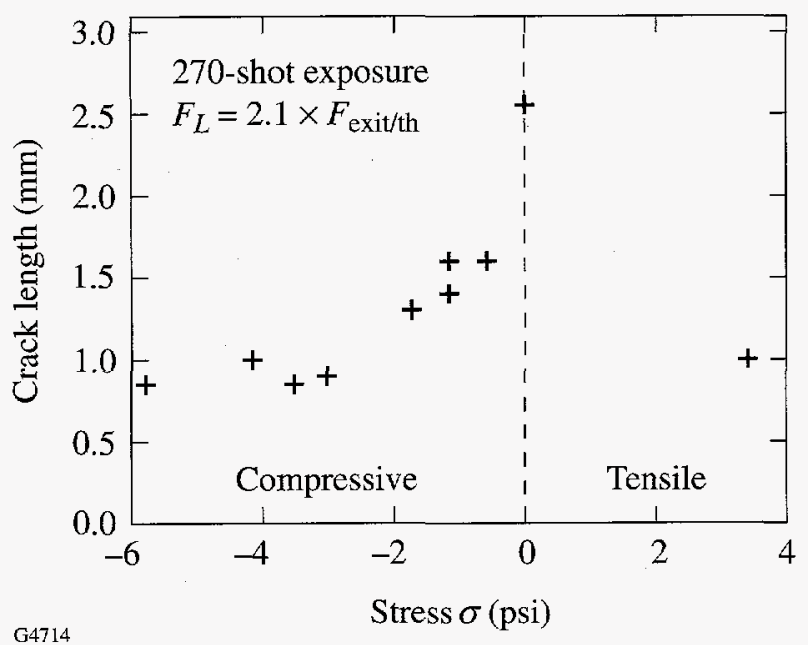

Figure 77.29

Crack length as a function of applied external stress for identical irradiation conditions as in Fig. 77.28.

\section{ACKNOWLEDGMENT}

This project was funded by the U.S. Department of Energy under Cooperative Agreement No. DE-FC03-92SF19460, the University of Rochester, and the New York State Energy Research Development Authority. The support of DOE does not constitute an endorsement of the views expressed in this article. One of the authors thanks the Laboratory for Laser Energetics for an F. J. Horton Fellowship. We thank Alex Maltsev for masterfully polishing all sample faces and Tom Greene for loan of the load cell.

\section{REFERENCES}

1. F. Dahmani, J. C. Lambropoulos, A. W. Schmid, S. Papernov, and S. J. Burns, "Fracture of Fused Silica with 351-nm-Laser-Generated Surface Cracks," to be published in Journal of Materials Research.

2. F. Dahmani, A. W. Schmid, J. C. Lambropoulos, and S. J. Burns, Appl. Opt. 37, 7772 (1998).

3. ANSYS $5.4{ }^{3}$ is a finite-element code developed by Ansys Inc.

4. N. L. Boling, M. D. Crisp, and G. Dubé, Appl. Opt. 12, 650 (1973).

5. H. Yokota et al., Surf. Sci. 16, 265 (1969). 


\section{Theory of the Ablative Richtmyer-Meshkov Instability}

In inertial confinement fusion (ICF) implosions, a laser irradiation induces a shock wave propagating through the target. During the shock transit time, the ablation front travels at a constant velocity, and any surface perturbations could grow due to the Richtmyer-Meshkov (RM)-like instability. ${ }^{1-5}$ Later, when a rarefaction wave reaches the ablation surface, the acceleration of the interface becomes finite, and ablationfront perturbations (multiplied by the RM growth) grow due to the Rayleigh-Taylor (RT) instability. It is important to study the perturbation evolution during the shock transit time mainly for two reasons: (1) to determine the initial conditions for the RT phase of instability and (2) to analyze the level of laser imprint on directly driven ICF targets.

The RM instability occurs when a plane shock interacts with a corrugated interface between two fluids (see Fig. 77.30). As a result of such an interaction, interface perturbation starts to grow because the transmitted shock is converging at the peak (point A) and diverging at the valley (point B). Converging shock increases pressure and accelerates perturbation peak into fluid 2. Similar instability occurs at the distorted interface of an ablatively driven target, where ablation pressure generates a rippled shock that induces pressure perturbation at the ablation front and causes distortion growth. The classical treatment of the RM problem leads to a linear-in-time asymptotic perturbation growth ${ }^{1} \eta\left(k c_{s} t>1\right) \simeq \eta_{0} k c_{s} t$, where $\eta$ is the interface perturbation, $k$ is the mode wave number, $c_{s}$ is the sound speed of the compressed material, and $\eta_{0}$ is a constant depending on the initial conditions. Recent studies showed that the ablation of material from the target surface turns such a growth into damped oscillations. ${ }^{4,5}$ During the last two years, several researchers have made attempts to develop an analytic theory of the ablative Richtmyer-Meshkov instability. In Refs. 4 and 5, the authors, on the basis of a gas dynamic model, found saturation of perturbations. At the ablation front, however, they used an heuristic boundary condition that, as will be shown later, contradicts the result of the self-consistent theory. ${ }^{6-8}$ In Ref. 3 the boundary conditions at the ablation front were derived by using the Chapman-Jouget deflagration model. As criticized in Ref. 5, however, this
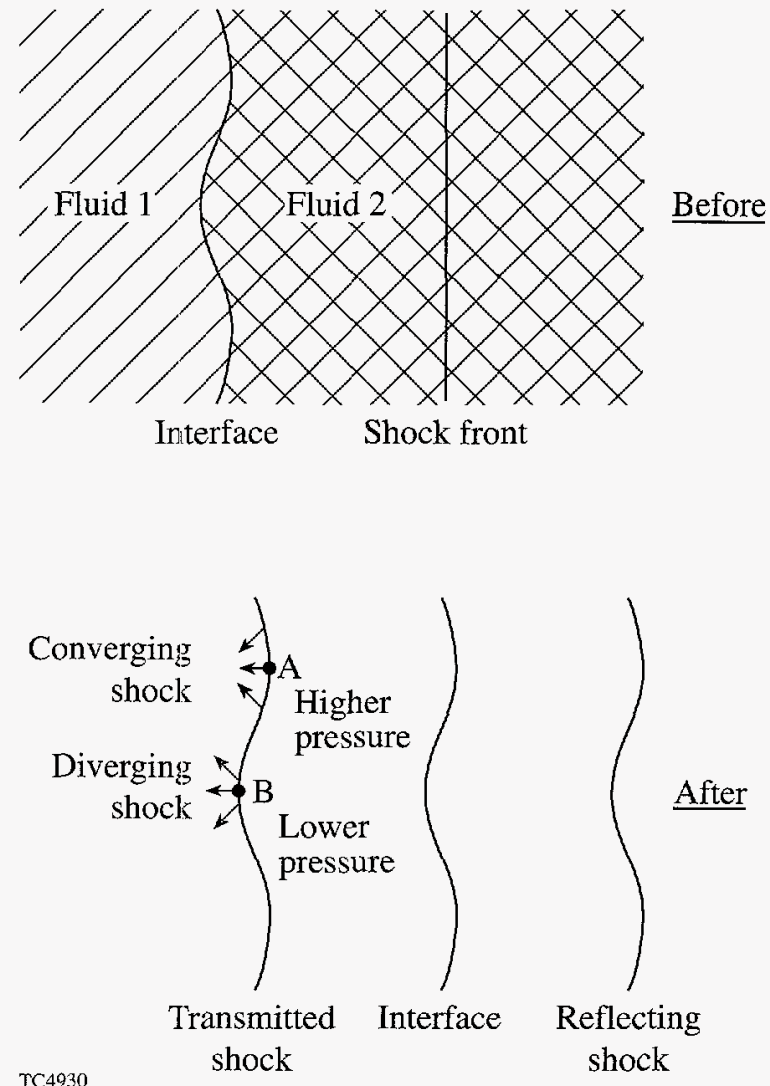

Figure 77.30

Richtmyer-Meshkov instability occurs when a plane shock interacts with a corrugated interface between two fluids.

model does not give an adequate description of the ablative process. In this article, we develop a sharp-boundary model to study the imposed mass-perturbation growth during the shocktransit time. The boundary conditions at the shock front are derived using the Hugoniot relations. At the ablation front the result of the self-consistent analysis ${ }^{6-8}$ is applied, and it is shown that the asymptotic behavior of the ablation-front perturbations is quite different from the earlier theoretical predictions. ${ }^{3-5}$ In particular, the dynamic overpressure causes 
perturbation oscillations in time (in agreement with the numerical results) $)^{2,5}$ with the frequency $\omega=k \sqrt{V_{a} V_{\mathrm{bl}}}$ and the amplitude $\eta_{0} c_{s} / \sqrt{V_{a} V_{\mathrm{bl}}}$, where $V_{a}$ and $V_{\mathrm{bl}}$ are the ablation and blow-off velocity, respectively. In addition, the mass ablation damps the oscillation amplitude on a time scale $1 /\left(k V_{a}\right)>1 / \omega$.

To study the linear perturbation growth during the shocktransit time, we consider a sharp-boundary model and identify the following three constant-density regions (see Fig. 77.31): (1) uncompressed material (undriven portion of the target) $y<$ $y_{s}\left(\rho=\rho_{1}\right)$, (2) material compressed by the shock $y_{s}<y<y_{a}$ $\left(\rho=\rho_{2}\right)$, and (3) ablated plasma $y>y_{a}$ with the density $\rho=\rho_{3}$. In the ablation-front frame of reference, the compressed material and blowoff plasma are moving in a positive $y$ direction with velocities $V_{a}$ and $V_{\mathrm{bl}}=V_{a} \rho_{2} / \rho_{3}$, respectively. In the shock-front frame of reference, the undriven- and compressedfluid velocities are $U_{1}=\sqrt{\rho_{2} / \rho_{1}\left(P_{2}-P_{1}\right) /\left(\rho_{2}-\rho_{1}\right)}$ and $U_{2}=$ $\rho_{1} U_{1} / \rho_{2}$, where $P_{1(2)}$ is the pressure in the region 1(2).

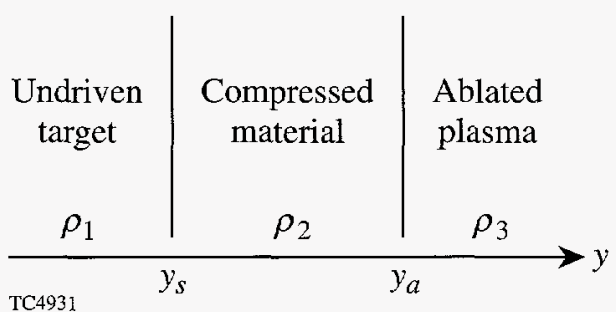

Figure 77.31

The equilibrium configuration is represented by the three regions (1) undriven target, (2) compressed material, and (3) ablated plasma

The stability analysis of the described equilibrium is performed in the standard fashion. First, all perturbated quantities are decomposed in the Fourier space $Q_{1}=\tilde{Q}(y, t) e^{i k x}$. Then, in the frame of reference moving with the compressed-region velocity, the linearized conservation equations are combined into a single partial differential equation for the pressure perturbation $\tilde{p}: 1,3,9$

$$
\partial_{t}^{2} \tilde{p}-c_{s}^{2} \partial_{y}^{2} \tilde{p}+k^{2} c_{s}^{2} \tilde{p}=0
$$

where $c_{s}$ is the sound speed of the compressed material. The boundary conditions at the ablation front can be derived by integrating the perturbed conservation equations across the interface $y=y_{\alpha}$. The result is

$$
\begin{aligned}
& \frac{\tilde{v}_{x}-\tilde{v}_{x}^{l}}{c_{s}}=i \zeta_{a} \Omega(1-\delta),(1-\delta) d_{\tau} \zeta_{a}=\tilde{v}_{y}-\delta \tilde{v}_{y}^{l}, \\
& \tilde{w}-\tilde{w}^{l}=2 \frac{V_{a}}{c_{s}} \Omega(1-\delta) \zeta_{a},
\end{aligned}
$$

where superscript $l$ denotes the blowoff region variable, $\Omega=$ $V_{\mathrm{bl}} / c_{s}, \delta=\rho_{3} / \rho_{2}, \tau=k c_{s} t, \tilde{w}=\tilde{p} /\left(\rho_{2} c_{s}^{2}\right)$, and $\zeta_{a}=k \eta_{a}$ is the normalized ablation-surface perturbation. It is well known ${ }^{10,11}$ that the sharp-boundary model cannot be solved in closed form in the presence of ablation without a supplementary boundary condition at the surface of discontinuity. The closure equation can be derived only by using the self-consistent stability analysis of ablation fronts. In Refs. 6-8 such an analysis was carried out by keeping finite thermal conductivity in the energy-conservation equation. Taking the limit of zero ablation-front thickness in the analytical solution, one can derive the jump conditions for the hydrodynamic quantities at the ablation front. ${ }^{8}{ }^{10}$ In addition to the conditions (2), the following jump in the perturbed transverse velocity is found:

$$
\tilde{v}_{y}-\tilde{v}_{y}^{l}=-\zeta_{a} V_{\mathrm{bl}}(1-\delta)
$$

Observe that by combining Eqs. (2) and (3) one can derive an equation for the perturbation evolution $d_{t} \eta_{a}+k V_{a} \eta_{a}=\tilde{v}_{y}$ that contradicts the boundary condition chosen heuristically in Eq. (10) of Ref. 5. At the shock front the boundary conditions are obtained by using the perturbed Hugoniot relations. The details of calculation can be found in Ref. 9. Next, to simplify the matching procedure we introduce new variables $r=\sqrt{\tau^{2}-k^{2} y^{2}}$ and $\theta=\tanh ^{-1}(k y / \tau)$; then Eq. (1) and the boundary conditions at the shock and ablation fronts take the following form:

$$
\partial_{r}^{2} \tilde{w}+\frac{1}{r} \partial_{r} \tilde{w}+\tilde{w}-\frac{1}{r^{2}} \partial_{\theta}^{2} \tilde{w}=0
$$

$$
\tilde{w}_{s}=\frac{L_{1}}{\cosh \theta_{s}} d_{r} \zeta_{s}
$$

$$
\partial_{\theta} \tilde{w}_{s}=-r L_{3} \frac{\sinh ^{2} \theta_{s}}{\cosh \theta_{s}} \zeta_{s}-\frac{r L_{2}}{\cosh \theta_{s}} d_{r}^{2} \zeta_{s}
$$




$$
\begin{gathered}
(1-\delta) d_{r}^{2} \zeta_{a}-\delta \Omega^{2} \zeta_{a}+\frac{\partial_{\theta} \tilde{w}_{a}}{r}+\tilde{w}_{a}=O\left(\delta^{2}\right) \\
d_{r}\left(\delta \Omega d_{r} \zeta_{a}+\delta \Omega^{2} \zeta_{a}-\tilde{w}_{a}\right) \\
=\delta \Omega \tilde{w}_{a}+\delta^{2} \Omega^{2} F(r)+O\left(\delta^{3}\right)
\end{gathered}
$$

where $\tanh \theta_{a}=-V_{a} / c_{s}, \tanh \theta_{s}=-U_{2} / c_{s}, \tilde{w}_{s}=\tilde{w}\left(r, \theta_{s}\right)$, $\tilde{w}_{a}=\tilde{w}\left(r, \theta_{a}\right)$, and

$$
\begin{aligned}
L_{1} & =\frac{4}{\gamma+1} \tanh \theta_{s}, L_{2}=2 \frac{M_{1}^{2}+1}{M_{1}^{2}(\gamma+1)}, \\
L_{3} & =2 \frac{M_{1}^{2}-1}{2+(\gamma-1) M_{1}^{2}}, \\
F(r) & =\frac{1}{L_{1}}\left(\frac{4}{\gamma+1}-L_{2}-L_{3}\right) \tilde{w}\left(\frac{-\delta \Omega r}{\sinh \theta_{s}}, \theta_{s}\right) .
\end{aligned}
$$

Here $M_{1}=U_{1} / c_{1}$ is the shock Mach number, and $c_{1}$ is the sound speed of the undriven material. A general solution of Eq. (4) can be written as an infinite sum of Bessel functions $J_{\mu}(r): 3,9$

$$
\tilde{w}=\sum_{\mu}\left(M_{\mu} \cosh \mu \theta+N_{\mu} \sinh \mu \theta\right) J_{\mu}(r)
$$

where constants $M_{\mu}$ and $N_{\mu}$ are determined from the boundary conditions (5)-(8). The temporal evolution of the front-surface perturbations is described by Eqs. (7) and (8) that can be solved by using the multiple-scale analysis. Next, we introduce a long-scale variable $T=\sqrt{\delta} r$ and make the following ordering: $\Omega \sim 1,\left(V_{a} / c_{s}\right) \sim \delta \ll 1$. Also we assume that

$$
\tilde{w}_{a} \sim \delta, \partial_{r} \tilde{w}_{a} \sim \delta, \partial_{\theta} \tilde{w}_{a} \sim 1
$$

The last assumptions will be verified $a$ posteriori. The system (7)-(8) then reduces to

$$
\begin{aligned}
& \partial_{r}^{2} \zeta_{a}+2 \sqrt{\delta} \partial_{r T}^{2} \zeta_{a}+\delta \partial_{T}^{2} \zeta_{a}+\delta \Omega \partial_{r} \zeta_{a} \\
& +\delta^{3 / 2} \Omega \partial_{T} \zeta_{a}+\frac{\partial_{\theta} \tilde{w}_{a}}{r}=f(T),
\end{aligned}
$$

$$
\begin{aligned}
& \partial_{r}^{2} \zeta_{a}+2 \sqrt{\delta} \partial_{r T}^{2} \zeta_{a}+\Omega \partial_{r} \zeta_{a}+\sqrt{\delta} \Omega \partial_{T} \zeta_{a} \\
& +\Omega^{2} \zeta_{a}-\frac{\tilde{w}_{a}}{\delta}=f(T),
\end{aligned}
$$

where $f(T)$ satisfies the first-order differential equation

$$
d_{T} f(T)=\Omega \tilde{w}_{a} / \sqrt{\delta}+\sqrt{\delta} \Omega^{2} F(T)
$$

In Eqs. (11) and (12) the ablation-front perturbation $\zeta_{a}$ and constants $N_{\mu}$ and $M_{\mu}$ are expanded in powers of $\delta: Q=Q^{0}+$ $\delta Q^{1}+\ldots$. To the first order in $\sqrt{\delta}$, the solution of Eq. (11) is

$$
\zeta^{0}=A(T)+B r-\int_{0}^{r} d t \int_{0}^{t} d \xi\left(\frac{\partial_{\theta} \tilde{w}_{a}(\xi)}{\xi}\right)^{0}
$$

The constant $B$ and function $A(T)$ are determined from the matching conditions. The functions $M_{\mu}(T)$ can be found by solving Eqs. (11)-(13) and keeping only terms up to the order of $\sqrt{\delta}$. The result is $M_{i}^{0}=0, M_{0}^{1}=\Delta_{2}-N_{1}^{0} / 2$, and

$$
\begin{aligned}
M_{2 i}^{1}=2 \Delta_{2}-\frac{1}{2}[ & 4 \Omega^{2} \sum_{k=1}^{i}(2 k-1) N_{2(i-k)+1}^{0} \\
& \left.+N_{2 i-1}^{0}+N_{2 i+1}^{0}\right], i=1,2 \ldots
\end{aligned}
$$

$$
\begin{aligned}
M_{2 i+1}^{1}= & 2(2 i+1) B \Omega^{2} \\
& -2 \Omega\left[\sum_{k=0}^{i} N_{2 k+1}^{0}-(i+1) N_{2 i+1}^{0}\right], i=0,1 \ldots
\end{aligned}
$$

where $\Delta_{2}=\Omega \sqrt{\delta} A^{\prime}(T)+\Omega^{2} A(T)+\Omega B-f(T)$. Observe that Eqs. (14) and (15) confirm our initial assumption (10). Next, we derive an equation for the $\delta$ correction to the front perturbation. Keeping $\delta$ - and $\delta^{3 / 2}$-order terms in Eq. (11) yields

$$
\begin{aligned}
\partial_{r}^{2} \zeta^{1}= & -A^{\prime \prime}(T) \\
& -\Omega \sqrt{\delta} A^{\prime}(T)-\Omega \partial_{r} \zeta^{0}-\left(\frac{\partial_{\theta} \tilde{w^{\prime}}}{r}\right)^{1}+f(T) .
\end{aligned}
$$


Following the standard procedure of the multiple-scale analysis, we eliminate the secular terms in the last equation. This condition gives the following differential equation for $A(T)$ :

$$
A^{\prime \prime}(T)+\sqrt{\delta} \Omega A^{\prime}(T)+\Omega\left(B+C_{t}\right)+\left.\left(\frac{\partial_{\theta} \tilde{w}}{r}\right)^{1}\right|_{r \rightarrow \infty}=f(T),
$$

where

$$
\begin{aligned}
\left(\frac{\partial_{\theta} \tilde{w}}{r}\right)^{1}= & \frac{1}{2}[ \\
& N_{1}^{1} J_{0}(r)+N_{2}^{1} J_{1}(r) \\
& \left.\quad+\sum_{\mu=2}^{\infty} J_{\mu}(r)\left(N_{\mu-1}^{1}+N_{\mu+1}^{1}\right)\right],
\end{aligned}
$$

and $C_{t}=-\sum_{i=0}^{\infty} N_{2 i+1}^{0}$. The functions $N_{\mu}(T)$ can be found by solving Eqs. (5) and (6). After some straightforward algebra, in the limit of $\mu \gg>1$, we obtain $N_{\mu}^{1} \approx M_{\mu}^{1}$, and Eq. (18) becomes

$$
\left.\left(\frac{\partial_{\theta} \tilde{w}}{r}\right)^{1}\right|_{r \rightarrow \infty}=\Delta_{2}-C_{1} \Omega^{2}+\Omega C_{t}+\left(B+C_{t}\right) \Omega^{2} r,
$$

where $C_{1}=-\sum_{i=0}^{\infty}(2 i+1) N_{2 i+1}^{0}$. Eliminating the secular terms in Eq. (16) with the help of Eq. (19) gives $B=-C_{t}$, and

$$
A^{\prime \prime}+2 \sqrt{\delta} \Omega A^{\prime}+\Omega^{2} A=2 f(T)+\Omega^{2} C_{1} .
$$

Substituting Eq. (20) into Eq. (13) and using the fact that the coefficients $N_{i>3}^{0}$ are numerically small for an arbitrary Mach number $M_{1}$ yields

$$
\begin{aligned}
& \frac{\eta_{a}\left(k c_{s} t>1\right)}{\eta_{0}}=\Sigma_{3} e^{k V_{a} t} \int_{\infty}^{-k V_{a} t / \sinh \theta_{s}} e^{\eta \sinh \theta_{s}} J_{1}(\eta) d \eta \\
& +\left[\frac{c_{s} \Sigma_{2}}{\sqrt{V_{a} V_{\mathrm{bl}}}} \sin \omega t+\left(1-\Sigma_{1}+\Sigma_{4}\right) \cos \omega t\right] e^{-2 k V_{a} t},
\end{aligned}
$$

where $\omega=k \sqrt{V_{a} V_{\mathrm{bl}}}$ and

$$
\begin{aligned}
& \Sigma_{0}=\frac{16\left(M_{1}^{2}-1\right) M_{1}^{2}}{\left(2 \gamma M_{1}^{2}-\gamma+1\right)\left(3 M_{1}^{2}+1\right)}, \\
& \Sigma_{1}=2 \Sigma_{0} \frac{M_{1}^{4}(5 \gamma-1)+2 M_{1}^{2}(\gamma+3)-3 \gamma-1}{M_{1}^{4}(17 \gamma-7)+2 M_{1}^{2}(\gamma+9)-3 \gamma+5}, \\
& \Sigma_{2}=\frac{1}{3}\left(\Sigma_{0}+\Sigma_{1}\right), \\
& \Sigma_{3}=\frac{\sum_{0}}{L_{1}}\left(\frac{4}{\gamma+1}-L_{2}-L_{3}\right) \frac{\sinh ^{2} \theta_{s}}{\Omega}, \\
& \Sigma_{4}=\sum_{3}\left(1-\tanh \theta_{s}\right) .
\end{aligned}
$$

Equation (21) shows that the ablation-front perturbations oscillate in time with the frequency $\omega$ proportional to the ablation velocity $V_{a}$. In addition, the amplitude of oscillations is damped by the mass ablation [term $e^{-2 k V_{a} t}$ in Eq. (21)]. The period of oscillations is much smaller, however, than the damping rate $k V_{a} / \omega=\sqrt{V_{a} / V_{\mathrm{bl}}}=\sqrt{\delta} \ll 1$. In the limit of zero ablation velocity, $\sin \omega t \approx k \sqrt{V_{a} V_{\mathrm{b} 1}} t$, and Eq. (21) leads to a classical asymptotic linear growth $\eta\left(k c_{s} t>1\right) \approx$ $\eta_{0}\left(1-\Sigma_{1}+k c_{s} \Sigma_{2} t\right)$.

The oscillatory behavior of the perturbations can be explained on the basis of the following simple model. Let us consider a slab of a uniform-density fluid with the perturbed right interface. If the applied pressure at the left and right sides of the slab is $P_{L}$ and $P_{R}$, then the effective acceleration experienced by the slab is $g_{\text {eff }}=-\left(P_{R}-P_{L}\right) / M$, where $M=$ $\rho L$ is the mass of the slab and $L$ is its length. In the case where the effective acceleration is pointing in the direction from a perturbed interface toward the slab, such a configuration is hydrodynamically stable, and any surface perturbations oscillate in time (gravity wave). For a target driven by a laser irradiation, the dynamic pressure in the blowoff region $P_{R}=\rho_{3} V_{\mathrm{bl}}^{2}=\rho_{2} V_{a} V_{\mathrm{bl}}$ is greater than the dynamic pressure in the shock-compressed region $P_{L}=\rho_{2} V_{a}^{2}=P_{R} \delta<P_{R}$, and the effective acceleration is pointing in the direction of the density gradient (from the perturbed ablation surface toward the shock compressed region). From the equation describing the temporal evolution of a gravity wave $\ddot{\eta}=k g_{\text {eff }} \eta$, it follows 
that the frequency of oscillations is $\omega=\sqrt{-k g_{\text {eff }}} \simeq \sqrt{k V_{a} V_{\mathrm{bl}} / L}$, and we recover the result of Eq. (21) with $L=1 / k$. The simple model described above shows that the dynamic overpressure is the main stabilizing mechanism of the ablation-surface perturbations during the shock-transit time. The estimate of the oscillation frequency can be also obtained by using the result of the self-consistent theory of the ablative RT instability. ${ }^{6-8}$ For the large Froude number case (small acceleration), the perturbation growth rate is $\gamma \simeq \sqrt{k g-k^{2} V_{a} V_{\mathrm{bl}}}-2 k V_{a}$. Taking the limit of $g \rightarrow 0$ in the last expression gives the oscillation frequency $\omega=i \gamma=k \sqrt{V_{a} V_{\mathrm{bl}}}$, in agreement with Eq. (21).

For a quantitative comparison of the model [Eq. (21)] with the result of numerical simulations, one needs to estimate the value of blowoff velocity $V_{\mathrm{bl}}$. Simulations and the selfconsistent analysis of ablation fronts ${ }^{6-8}$ show that the velocity of ablated plasma is not uniform, and it increases in the direction toward the blowoff plasma. As shown in Refs. 7 , 8 , and 10 , however, the appropriate value of the blowoff velocity to be substituted into the sharp-boundary model is $V_{\mathrm{bl}} \equiv V_{a} / \delta=V_{a} /\left[\mu(v)\left(k L_{0}\right)^{1 / v}\right]$, where $v$ is the power index for the thermal conduction, $L_{0}$ is the characteristic thickness of ablation front (proportional to the minimum density-gradient scale length $),{ }^{7} \mu=(2 / v)^{1 / v} / \Gamma(1+1 / v)+0.12 / v^{2}$, and $\Gamma(x)$ is the gamma function. The effective power index $v$ and the thickness of the ablation front $L_{0}$ can be determined by fitting the hydrodynamic profiles obtained using the 1-D hydrodynamic code with the solution of the isobaric model. ${ }^{12}$ For plastic $(\mathrm{CH})$ targets directly driven by a flat-top laser pulse with an intensity of 50 to $200 \mathrm{TW} / \mathrm{cm}^{2}, L_{0} \simeq 0.1 \mu \mathrm{m}, v \simeq 1$, and the oscillation period is

$$
T_{\mathrm{CH}} \simeq 2.8 /\left[V_{a}(\mu \mathrm{m} / \mathrm{ns}) \sqrt{k\left(\mu \mathrm{m}^{-1}\right)}\right] \mathrm{ns} .
$$

Cryogenic DT targets have a much smaller density-gradient scale length $L_{0} \simeq 0.01 \mu \mathrm{m}, v \simeq 2$, and

$$
T_{\mathrm{DT}} \simeq 2 /\left[V_{a}(\mu \mathrm{m} / \mathrm{ns}) k^{3 / 4}\left(\mu \mathrm{m}^{-1}\right)\right] \mathrm{ns} .
$$

For the cryogenic NIF target designs, $V_{a} \simeq 2 \mu \mathrm{m} / \mathrm{ns}$ during the shock-transit time, and $T_{\mathrm{DT}}=2.5 \mathrm{~ns}$ for $20-\mu \mathrm{m}$ perturbation wavelength. In this case the ablation-front perturbations will experience several oscillations (the breakout time for such targets is around $5 \mathrm{~ns}$ ). Figure 77.32 shows the front-perturbation evolution of the $200-\mu$ m-thick DT foil driven by a square

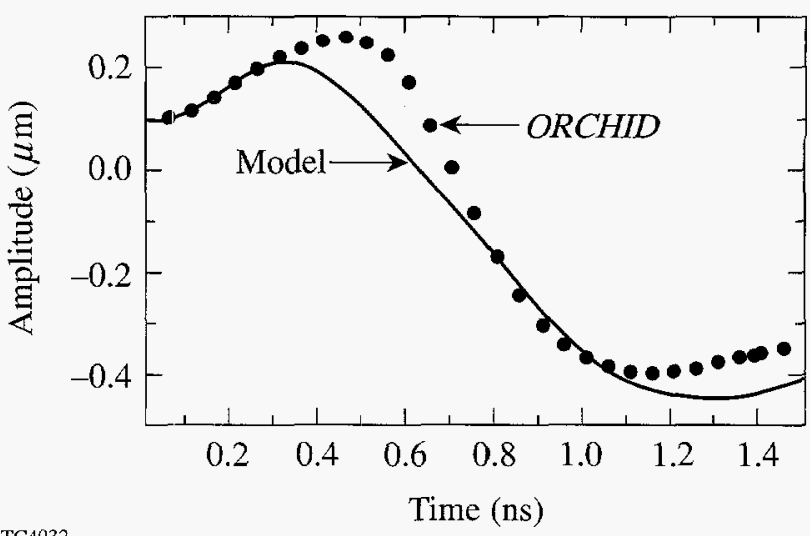

Figure 77.32

Time evolution of the ablation-front perturbation calculated using analytic formula (22) (solid line) compared with the numerical results (dots) of the 2-D hydrocode $O R C H I D$.

pulse with an intensity of $100 \mathrm{TW} / \mathrm{cm}^{2}$. The initial amplitude of perturbation is $0.1 \mu \mathrm{m}$, and its wavelength is $20 \mu \mathrm{m}$. The dots represent the result of 2-D hydrocode ORCHID, ${ }^{13}$ and the solid line shows the prediction of the sharp-boundary model. Observe that the analytic formula (21) reproduces not only the period of oscillation but also its amplitude.

In summary, the analytic theory of the ablative RichtmyerMeshkov instability was developed. It was shown that the main stabilizing mechanism of the ablation-front perturbations is the dynamic overpressure of the blowoff plasma with respect to a target material.

\section{ACKNOWLEDGMENT}

The author thanks Professors R. Betti and J. Sanz and Dr. C. Cherfils for helpful discussions. This work was supported by the U.S. Department of Energy Office of Inertial Confinement Fusion under Cooperative Agreement No. DE-FC03-92SF19460, the University of Rochester, and the New York State Energy Research and Development Authority. The support of DOE does not constitute an endorsement by DOE of the views expressed in this article.

\section{REFERENCES}

1. R. D. Richtmyer, Commun. Pure. Appl. Math. XIII, 297 (1960).

2. S. E. Bodner, D. G. Colombant, J. H. Gardner, R. H. Lehmberg, S. P. Obenschain, L. Phillips, A. J. Schmitt, J. D. Sethian, R. L. McCrory, W. Seka, C. P. Verdon, J. P. Knauer, B. B. Afeyan, and H. T. Powell, Phys. Plasmas 5, 1901 (1998).

3. R. Ishizaki and K. Nishihara, Phys. Rev. Lett. 78, 1920 (1997). 
4. R. J. Taylor et al., Phys. Rev. Lett. 79, 1861 (1997).

5. A. L. Velikovich et al., Phys. Plasmas 5, 1491 (1998).

6. J. Sanz, Phys. Rev. Lett. 73, 2700 (1994).

7. V. N. Goncharov, R. Betti, R. L. McCrory, P. Sorotokin, and C. P. Verdon, Phys. Plasmas 3, 1402 (1996).

8. V. N. Goncharov, "Self-Consistent Stability Analysis of Ablation Fronts in Inertial Confinement Fusion," Ph.D thesis, University of Rochester, 1998.
9. P. M. Zaidel, J. Appl. Math. Mech. 24, 316 (1960).

10. A. R. Piriz, J. Sanz, and L. F. Ibanez, Phys. Plasmas 4, 1117 (1997).

11. S. E. Bodner, Phys. Rev. Lett. 33, 761 (1974).

12. R. Betti, V. N. Goncharov, R. L. McCrory, and C. P. Verdon, Phys. Plasmas 5, 1446 (1998).

13. R. L. McCrory and C. P. Verdon, in Computer Applications in Plasma Science and Engineering, edited by A. T. Drobot (Springer-Verlag, New York, 1991). 


\section{Reverse Intersystem Crossing from a Triplet State of Rose Bengal Populated by Sequential 532-nm plus 1064-nm Laser Excitation}

Photodynamic therapy is a treatment in which the combination of a dye, light, and oxygen causes photochemically induced cell death. Observations of this effect occurred at least as early as the end of the last century. In 1900, Raab reported that the dye acridine rapidly killed paramecia when exposed to light, but had no effect in the dark. ${ }^{1}$ This observation quickly inspired attempts to use this effect to treat disease. Light and the dye eosin were combined to treat skin cancer in $1903 ;{ }^{2}$ however, significant progress in applying photodynamic therapy to the treatment of cancer did not occur until the 1940s and 1950s, when it was discovered that porphyrin-based photosensitive dyes preferentially accumulated in malignant tissues. ${ }^{3,4} \mathrm{~A}$ sustained series of studies into the mechanisms and applications of photodynamic therapy for the treatment of a broad range of cancers was initiated by Dougherty in the 1970s. ${ }^{5}$ This work led the U.S. Food and Drug Administration in December 1995 to approve the treatment of advanced esophageal cancer using photodynamic therapy with Photofrin ${ }^{\circledR}$, a porphyrin-based photosensitizer. In 1998 this approval was extended to cover the treatment of early-stage lung cancer. Other countries have also approved photodynamic therapy for the treatment of bladder, gastric, and cervical cancers. Several review articles have been published that provide an overview of the clinical results as well as the open questions about this therapy that require further research. 6,7

The photodynamic effect is a result of three primary processes. First, the ground state of the dye $\left(S_{0}\right)$ is optically excited to produce the excited singlet state $\left(\mathrm{S}_{1}\right)$. Population from this excited state is transferred by intersystem crossing (a radiationless transition) to the dye's lowest triplet state $\left(\mathrm{T}_{1}\right)$. Finally, collisional energy transfer from the triplet dye to ground-state molecular oxygen $\left({ }^{3} \mathrm{O}_{2}\right)$ produces highly reactive singlet oxygen $\left({ }^{1} \mathrm{O}_{2}\right)$ and returns the dye to its ground state:

$$
\begin{aligned}
& \mathrm{S}_{0}+h v \rightarrow \mathrm{S}_{1} \\
& \mathrm{~S}_{1} \rightarrow \mathrm{T}_{1} \\
& \mathrm{~T}_{1}+{ }^{3} \mathrm{O}_{2} \rightarrow \mathrm{S}_{0}+{ }^{1} \mathrm{O}_{2} .
\end{aligned}
$$

The singlet oxygen produced as a result of this three-step process reacts readily with many biological targets and, in sufficient quantity, can destroy a wide variety of cells.

Since the cell damage produced by conventional photodynamic therapy is due to singlet oxygen, an abundant supply of oxygen is critical for an effective treatment. One factor leading to oxygen depletion is the consumption of oxygen by the photodynamic process at a rate faster than it can be resupplied by the circulatory system. In addition, some tumors are inherently poorly oxygenated. A possible solution to this limitation has been suggested by observations that several dyes produce oxygen-independent damage following population of their higher-lying states. It is believed that these states contain sufficient energy to allow for the cleavage of one of the molecular bonds, producing radicals that are even more reactive than singlet oxygen. The production of these radicals does not require the presence of oxygen. Understanding this process requires greater knowledge of the properties of these higherlying states. In this article we report on our studies of rose bengal, a dye that has been found to produce oxygen-independent damage following excitation of one of its higher-lying triplet states. ${ }^{8}$ In particular, we have studied a decay mechanism based on reverse intersystem crossing from high-lying triplet states that may compete with the bond-cleavage process.

Although intersystem crossing has been identified primarily with transitions from the lowest excited singlet state of a molecule to an even lower-lying triplet state, triplet to singlet intersystem crossing also may occur. Well-known examples of reverse (tripler to singlet) intersystem crossing include $\mathrm{E}$ - and P-type delayed fluorescence. ${ }^{9}$ E-type delayed fluorescence, also known as delayed thermal fluorescence, is observed when thermal activation causes population transfer from $T_{1}$ back to the more-energetic $S_{1}$ state. The strength of E-type delayed fluorescence is temperature dependent, and its lifetime reflects that of $T_{1}$. P-type delayed fluorescence results when the activation energy is provided by triplet-triplet annihilation $\left(T_{1}\right.$ $+\mathrm{T}_{1} \rightarrow \mathrm{S}_{1}+\mathrm{S}_{0}$ ). The strength of the P-type delayed fluorescence increases quadratically with the triplet concentration. 
Reverse intersystem crossing may also occur from higherlying triplet states where intersystem crossing to the singlet manifold competes with direct internal conversion to the lowest triplet state. This process of reverse intersystem crossing from higher-lying triplets is responsible for two-step laserinduced fluorescence (TSLIF) observed in several dyes. ${ }^{10-13}$ The quantum yield of reverse intersystem crossing, $\Phi_{\text {risc }}$, can be quite small $\left(<10^{-5}\right),{ }^{10}$ but there are reports of exceptionally high yields $\left(\Phi_{\text {risc }}>0.1\right)$ in 9,10 -dibromoanthracence, ${ }^{11}$ several merocyanine derivatives, ${ }^{12}$ tetraphenylporphyrin, ${ }^{13}$ erythrosin $\mathrm{B},{ }^{13}$ and rose bengal. ${ }^{13}$

Several reports of reverse intersystem crossing in rose bengal (RB) have been published. ${ }^{13-15}$ Durán and Cilento ${ }^{14}$ describe observations of fluorescence following generation of RB triplets by energy transfer from excited triplet acetone. It was believed that higher-lying triplets were populated through triplet-triplet excitation transfer and subsequently relaxed to $\mathrm{S}_{1}$ through reverse intersystem crossing. The magnitude of the emission was compared for a series of xanthene dyes (fluorescein, eosin, and rose bengal), which revealed that heavy-atom substitution enhanced the effect. This process was not associated with a particular triplet state, and no attempt was made to quantify its yield. Ketsle et al. ${ }^{15}$ investigated transient absorption changes following two-pulse excitation $(532 \mathrm{~nm}+$ $694 \mathrm{~nm}$ ) of various fluorescein derivatives, including rose bengal, incorporated in polymer hosts. Photobleaching of the $\mathrm{T}_{1}$ absorption due to the second pulse was observed to have a component that was irreversible on the microsecond time scale. It was observed that the decrease in concentration of $T_{1}$ equaled the increase in concentration of $\mathrm{S}_{0}$, providing evidence for a photophysical rather than photochemical process. Fluorescence emission was also observed coincident with the second pulse. A reverse intersystem crossing quantum yield of 0.72 was reported for $T_{3}$, the triplet state excited by red light. Most recently, the work of Reindl and Penzkofer ${ }^{13}$ reported an $80 \%$ quantum yield of reverse intersystem crossing for $\mathrm{T}_{4}$, the state excited through absorption of green light by $\mathrm{T}_{1}$. Using a model of the population dynamics, the yield was extracted from measurements of the pulse-to-pulse variation in fluorescence for a train of picosecond pulses.

The present work is the first study to identify and investigate the properties of $T_{2}$, a triplet state in rose bengal populated by near-infrared light $(\lambda=1064 \mathrm{~nm})$. We have used laser flash photolysis and two-step laser-induced fluorescence measurements to determine the triplet-triplet absorption crosssection spectrum in the near infrared and the quantum yield of reverse intersystem crossing and lifetime of $T_{2}$. In addition, upper limits on the reverse intersystem crossing yield for $T_{3}$ are established.

\section{Experimental}

The foundation of the laser system is a mode-locked Nd:YAG laser that generates a train of pulses at $76 \mathrm{MHz}$. Every $400 \mathrm{~ms}$ a single pulse is selected using an electro-optic switch and amplified using a regenerative amplifier followed by a flashlamp-pumped two-pass amplifier (both Nd:YAG). The amplified pulses have a wavelength of $1064 \mathrm{~nm}$, a pulse length of $\sim 190 \mathrm{ps}$, and energies exceeding $2 \mathrm{~mJ}$. The second harmonic is generated from this pulse using a KDP crystal, resulting in a pulse with a wavelength of $532 \mathrm{~nm}$, a pulse length of $\sim 134 \mathrm{ps,}$ and an energy greater than $250 \mu \mathrm{J}$.

The experimental setup for the laser flash photolysis measurements is shown in Fig. 77.33 using the symbols defined in Table 77.II. In this configuration the second-harmonic pulse $(\lambda$ $=532 \mathrm{~nm}$ ) is separated from the fundamental by a dichroic mirror (DM) with any residual light at the fundamental wavelength $(\lambda=1064 \mathrm{~nm})$ further attenuated by a filter $(F)$. The majority of this frequency-doubled pump pulse is focused by a cylindrical lens (CL) onto the masked sample cuvette, exciting a $2-\mathrm{mm}$ by $1-\mathrm{cm}$ cross-sectional area. A small fraction of the pump pulse is reflected by a glass plate (BS) prior to the cylindrical lens, attenuated by neutral density filters, and then detected by silicon photodiode PD1. This signal is captured by gated integrator GI1 and transferred to a computer. By removing the sample cuvette and placing an energy meter behind the beam mask, the pump pulse monitor signal measured by PD1 can be calibrated with respect to the energy reaching the

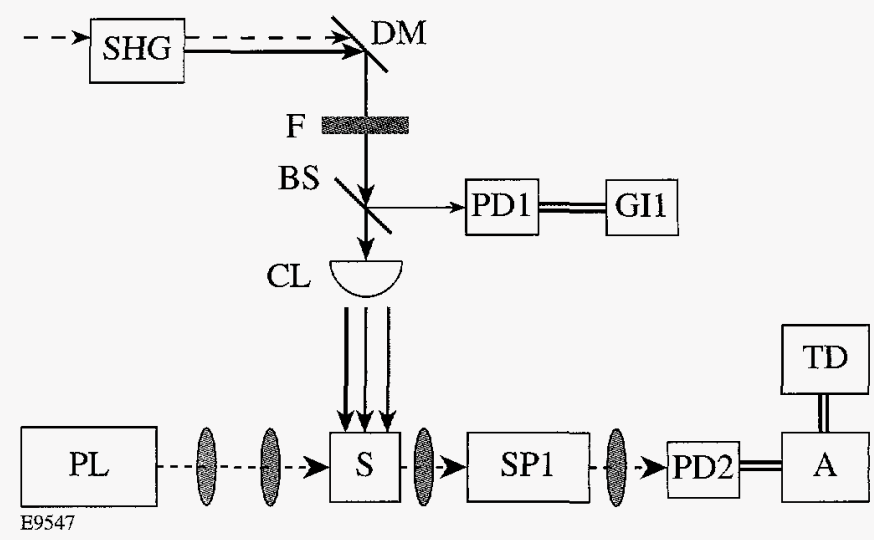

Figure 77.33

Experimental setup for laser flash photolysis measurements. See Table 77.II for symbol definitions. 
Table 77.II: Equipment used in the laser flash photolysis and two-step laser-induced fluorescence experiments.

\begin{tabular}{|c|l|l|}
\hline \hline & \multicolumn{1}{|c|}{ Description } & \multicolumn{1}{c|}{ Manufacturer, Model } \\
\hline A & Fast ampifier & EG\&G, 574 \\
\hline BS & Glass plate (microscope slide) & \\
\hline CL & Cylindrical lens & \\
\hline DM & Dichroic mirror, R @ 532 nm, T@ 1064 nm & \\
\hline F & Short-pass filter & Schott, KG3 \\
\hline GI1-4 & Gated integrator & Stanford Research Systems, 250 \\
\hline L1-3 & Lenses & \\
\hline P & Prism & \\
\hline PD1-2 & Silicon photodiodes & EG\&G, FND-100 \\
\hline PL & Mercury lamp & Vincent Associates, Uniblitz VS25 \\
& Fast shutter & Schott, RG695 \\
\hline PMT & Photomultiplier tube & Burle, 6199 \\
\hline PS & Polarizing beam splitter & \\
\hline S & Sample cuvette and beam mask & \\
\hline SHG & Second-harmonic generator $(\mathrm{KDP}$ crystal) & \\
\hline SP1 & Monochromator, bandwidth $\sim 13 \mathrm{~nm}$ & Instruments SA, H20 \\
\hline SP2 & Monochromator, bandwidth $\sim \mathrm{nm}$ & Photon Technology Intl., 102 \\
\hline TD & Digitizing oscilloscope & Hewlett-Packard, HP54201A \\
\hline WP & Half-wave plate $(\lambda=1064 \mathrm{~nm})$ & \\
\hline \hline
\end{tabular}

sample. Transient absorption changes are probed by a broadband light beam traveling along the length of the irradiated zone (perpendicular to the pump pulse). The probe pulse has a 20 -ms duration and is produced by a mercury lamp followed by a long-pass filter and a fast mechanical shutter. This collection of elements is represented by PLin Fig. 77.33. The probe pulse passes through monochromator SP1 before being detected by silicon photodiode PD2. The photodiode signal is increased by multistage amplifier A and then recorded by digital oscilloscope TD. The average signal from 64 shots at 9-bit resolution is then transferred to a computer for analysis.

The two-step laser-induced fluorescence measurements probing $\mathrm{T}_{2}$ are made with the optical layout shown in Fig. 77.34. To achieve a high degree of spectral separation between the fundamental and second-harmonic pulses, prism $P$ is used to spatially disperse the two beams. The first pump pulse (P1) has a wavelength of $532 \mathrm{~nm}$, and the second pump pulse (P2) has a wavelength of $1064 \mathrm{~nm}$. P2 is delayed by 34 ns relative to $P 1$ by traversal of a greater optical path length.
The delay path includes a half-wave plate followed by a polarizing beam splitter, allowing for continuous variation of the second pump pulse energy. The pump pulses, $\mathrm{P} 1$ and $\mathrm{P} 2$, are recombined spatially at dichroic mirror DM. The pulses pass through two pinholes, ensuring collimation, before irradiating a 2-mm-diam spot at the sample cuvette. As in the laser flash photolysis layout, a small fraction of the excitation light is reflected by a glass plate to a silicon photodiode. This signal is split before sampling by two gated integrators, GI1 and GI2, which distinguish between the $\mathrm{P} 1$ and $\mathrm{P} 2$ signals. The pumppulse signals are calibrated individually using an energy meter. Emission from the excited sample is collected, spectrally resolved using monochromator SP2, and detected by a photomultiplier tube. The signal from the PMT is split and sampled by the gated integrators GI3 and GI4. The temporal gate of GI3 is centered on the fluorescence excited by P1. The center of the GI4 temporal gate is set to be $34 \mathrm{~ns}$ later than the center of the GI3 gate, corresponding to the time delay between the pump pulses. Both gates are $20 \mathrm{~ns}$ wide. The values of all four gated integrators are recorded by a computer for each shot. 


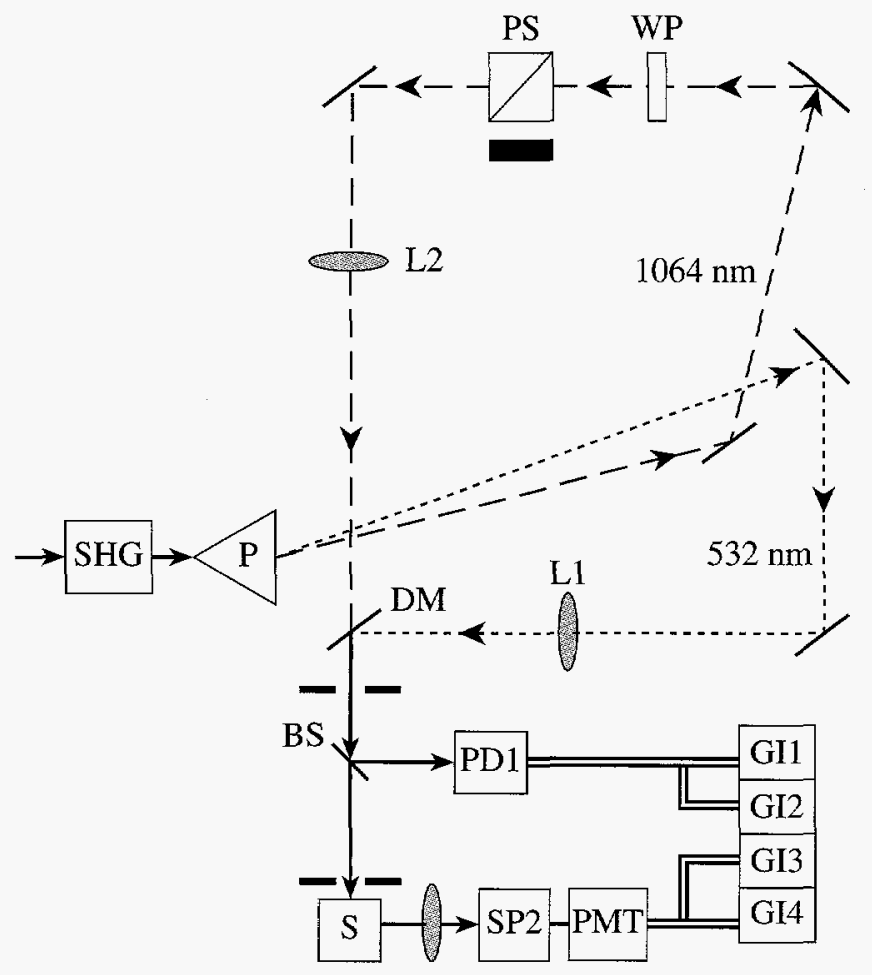

E9548

L3

Figure 77.34

Experimental setup for two-step laser-induced fluorescence measurements. See Table 77.II for symbol definitions.

Two-step laser-induced fluorescence measurements probing $T_{3}$ are made using a similar setup. In this case $P 2$, the 1064-nm pump pulse, is replaced by a 632-nm-wavelength pump pulse, while the first pump pulse remains at $532 \mathrm{~nm}$. The $632-\mathrm{nm}$ pulse is generated by stimulated Raman scattering of the Nd:YAG second harmonic in an 18-cm ethanol cell, resulting in $60 \mu \mathrm{J} / \mathrm{pulse}$ with a pulse length of approximately $80 \mathrm{ps}$. The 632-nm light is separated from the 532-nm light by a pair of prisms before P2 enters the delay line. The pulses are spatially recombined at the dichroic mirror DM, and from this point the system is identical to the previously described twostep, laser-induced fluorescence apparatus.

Rose bengal was purchased from Sigma (St. Louis) and used without further purification. All experiments were carried out in phosphate-buffered saline with a $\mathrm{pH}$ of 7 . Effects of photobleaching were minimized by continuously stirring all samples with a micro-stirbar during irradiation. Photobleaching was monitored by measuring the decrease in fluorescence as a function of the number of excitation pulses. There was a lessthan-5\% decrease in fluorescence after more than 3700 two- step excitations. Samples had a concentration of approximately $20 \mu \mathrm{M}$ and were stored in the dark prior to use.

\section{Analysis}

Several photophysical parameters associated with an upper triplet state can be determined from two-step laser-induced fluorescence (TSLIF) measurements collected over a range of second pump-pulse (P2) fluences. These measurements are sensitive to the lifetime of the upper triplet state excited by P2, the quantum yield of intersystem crossing from this state back to the singlet manifold, and its thermalization rate. The upper triplet photophysical parameters are determined by fitting a model of the two-step laser-induced fluorescence process to the fluence-dependent TSLIF data.

The kinetic model used to analyze the TSLIF experiments is shown in Fig. 77.35(a). The rate equations describing this model are

$$
\begin{aligned}
\frac{d p_{\mathrm{S}_{0}}}{d t}= & -\sigma_{\mathrm{S}_{0} \mathrm{~S}_{1}}\left(p_{\mathrm{S}_{0}}-p_{\mathrm{S}_{1^{\prime}}}\right) I_{1}(t) \\
& +\left(1-\Phi_{\mathrm{isc}}\right) \tau_{\mathrm{S}_{1}}^{-1} p_{\mathrm{S}_{1}}+\tau_{\mathrm{T}_{1}}^{-1} p_{\mathrm{T}_{1}},
\end{aligned}
$$

$$
\begin{aligned}
\frac{d p_{\mathrm{T}_{1}}}{d t}= & \Phi_{\mathrm{isc}} \tau_{\mathrm{S}_{1}}^{-1} p_{\mathrm{S}_{1}}-\tau_{\mathrm{T}_{1}}^{-1} p_{\mathrm{T}_{1}}-\sigma_{\mathrm{T}_{1} \mathrm{~T}_{4}}\left(p_{\mathrm{T}_{1}}-p_{\mathrm{T}_{4}}\right) I_{1}(t) \\
& -\sigma_{\mathrm{T}_{1} \mathrm{~T}_{n}}\left(p_{\mathrm{T}_{1}}-p_{\mathrm{T}_{n}^{\prime}}\right) I_{2}(t) \\
& +\left(1-\Phi_{\text {risc, } \mathrm{T}_{4}}\right) \tau_{\mathrm{T}_{4}}^{-1} p_{\mathrm{T}_{4}}+\left(1-\Phi_{\text {risc, } \mathrm{T}_{n}}\right) \tau_{\mathrm{T}_{n}}^{-1} p_{\mathrm{T}_{n}},
\end{aligned}
$$

$$
\frac{d p_{\mathrm{S}_{1}}}{d t}=k_{r} p_{\mathrm{S}_{1^{\prime}}}-\tau_{\mathrm{S}_{1}}^{-1} p_{\mathrm{S}_{1}}
$$

$$
\begin{aligned}
\frac{d p_{\mathrm{S}_{1^{\prime}}}}{d t}= & \sigma_{\mathrm{S}_{0} \mathrm{~S}_{1}}\left(p_{\mathrm{S}_{0}}-p_{\mathrm{S}_{1^{\prime}}}\right) I_{1}(t)-k_{r} p_{\mathrm{S}_{1^{\prime}}} \\
& +\Phi_{\text {risc, }, \mathrm{T}_{4}} \tau_{\mathrm{T}_{4}}^{-1} p_{\mathrm{T}_{4}}+\Phi_{\text {risc, } \mathrm{T}_{n}} \tau_{\mathrm{T}_{n}}^{-1} p_{\mathrm{T}_{n}},
\end{aligned}
$$

$$
\begin{aligned}
& \frac{d p_{\mathrm{T}_{n}}}{d t}=k_{r, \mathrm{~T}_{n}} p_{\mathrm{T}_{n}^{\prime}}-\tau_{\mathrm{T}_{n}}^{-1} p_{\mathrm{T}_{n}}, \\
& \frac{d p_{\mathrm{T}_{n}^{\prime}}}{d t}=\sigma_{\mathrm{T}_{1} \mathrm{~T}_{n}}\left(p_{\mathrm{T}_{1}-} p_{\mathrm{T}_{n}^{\prime}}\right) I_{2}(t)-k_{r, \mathrm{~T}_{n}} p_{\mathrm{T}_{n}^{\prime}}, \\
& \frac{d p_{\mathrm{T}_{4}}}{d t}=\sigma_{\mathrm{T}_{1} \mathrm{~T}_{4}}\left(p_{\mathrm{T}_{1}}-p_{\mathrm{T}_{4}}\right) I_{1}(t)-\tau_{\mathrm{T}_{4}}^{-1} p_{\mathrm{T}_{4}},
\end{aligned}
$$


where the $p_{i}^{\prime}$ 's are the populations of $\mathrm{S}_{0}, \mathrm{~T}_{1}, \mathrm{~S}_{1}, \mathrm{~S}_{1}^{\prime}, \mathrm{T}_{n}$, $\mathrm{T}_{n}^{\prime}$, and $\mathrm{T}_{4}$ (arranged in order of increasing energy), where $n=2$ or 3 . Table 77.II lists the definitions and values of the photophysical parameters. The unknown parameters are $\Phi_{\text {risc, } \mathrm{T}_{n}}, \tau_{\mathrm{T}_{n}}$, and $k_{r, \mathrm{~T}_{n}}$. The pump pulses $\mathrm{P} 1$ and $\mathrm{P} 2$ have a Gaussian temporal profile such that

$$
I_{1}(t)=\frac{F_{1}}{\sqrt{2 \pi \delta_{1}^{2}}} \exp \left[-(t+\Delta / 2)^{2} /\left(2 \delta_{1}^{2}\right)\right]
$$

and

$$
I_{2}(t)=\frac{F_{2}}{\sqrt{2 \pi \delta_{2}^{2}}} \exp \left[-(t-\Delta / 2)^{2} /\left(2 \delta_{2}^{2}\right)\right]
$$

where $F_{1}$ and $F_{2}$ are the fluences, $\delta_{1}$ and $\delta_{2}$ are related to the full-width-at-half-maximum pulse lengths by FWHM $=\sqrt{8 \ln 2} \delta$, and $\Delta$ is the time delay between the peaks of $\mathrm{P} 1$ and $\mathrm{P} 2$.

Excited-state absorption from states other than $T_{1}$ has been neglected. Previous experiments have found no evidence for absorption of 532-nm light by $\mathrm{S}_{1} \cdot{ }^{16}$ The state $\mathrm{T}_{4}$ may absorb 532-nm light and thus populate an even higher-lying state, but we assume with Reindl and Penzkofer ${ }^{13}$ that any such extremely high-lying state will relax back to $\mathrm{T}_{4}$ immediately. This process would affect transmission measurements, but since the present studies are concerned only with emission, it appears reasonable to omit it in this case. Finally, absorption by $\mathrm{T}_{n}$ is also not included in this model. The validity of this assumption will be discussed in the Results section.

As a result of the large time delay between the pump pulses ( $\Delta=34 \mathrm{~ns}$ ), it is possible to separate the system of rate equations $[(\mathrm{Eq}$. (1)] into two subsets. The set of equations describing the effects of the first pump pulse is (a)

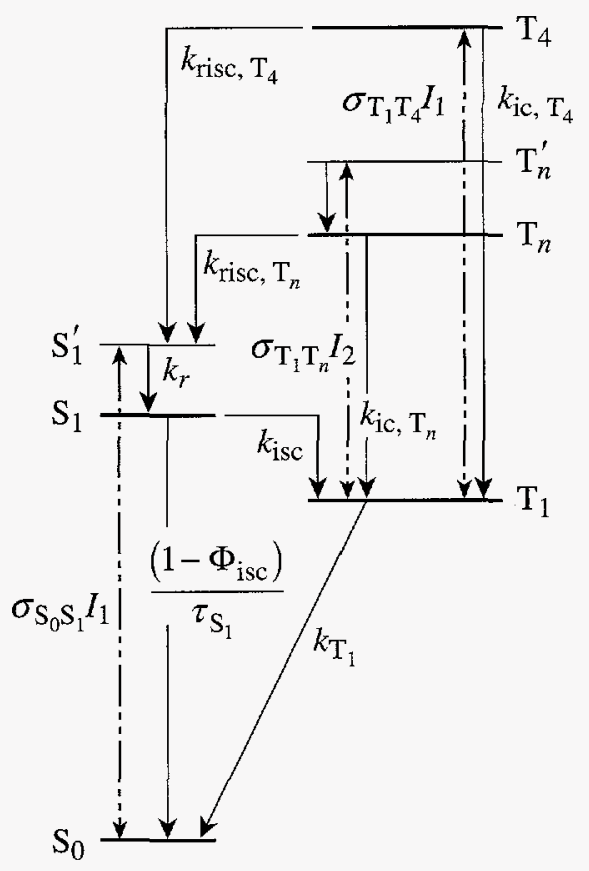

(b)

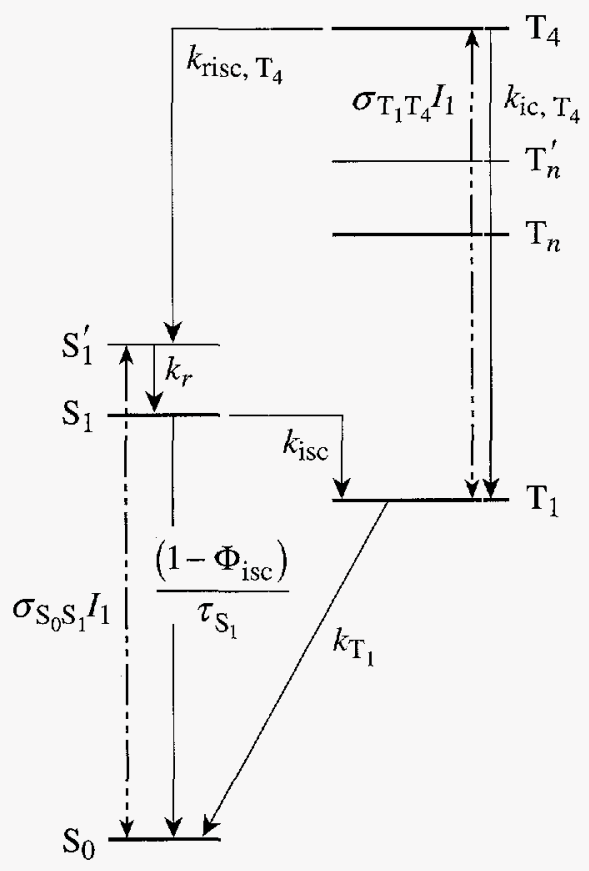

(c)

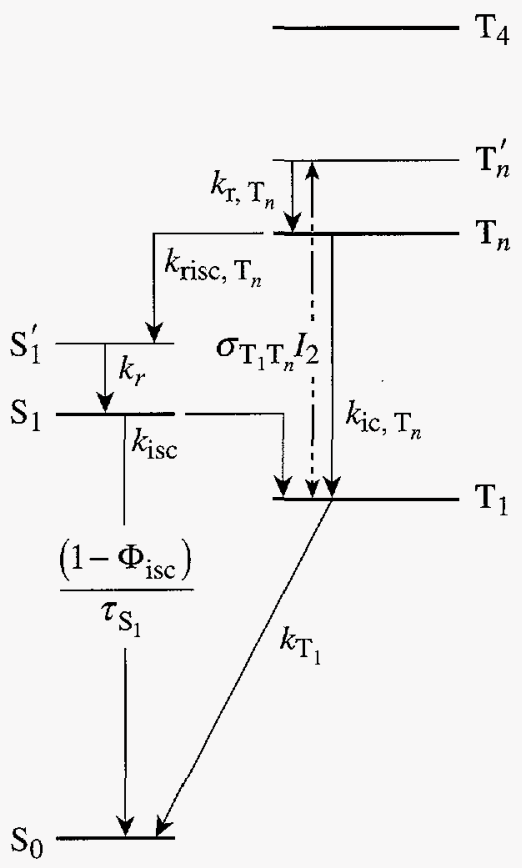

E9549,50,51

Figure 77.35

Energy-level scheme for description of two-color excitation dynamics. (a) Complete two-step model, (b) P1 subset of model, (c) P2 subset of model. See Table 77.III for parameter descriptions and values. 
Table 77.III: Parameters used in the two-step laser-induced fluorescence model for rose bengal.

\begin{tabular}{|c|c|c|c|}
\hline Parameter & Description & Value & Reference \\
\hline$\sigma_{\mathrm{S}_{0} \mathrm{~S}_{1}}$ & $\begin{array}{l}\text { Ground-state absorption cross section at } 532 \mathrm{~nm} \\
\left(\mathrm{~S}_{0}+\hbar \omega \rightarrow \mathrm{S}_{1}^{\prime}\right)\end{array}$ & $1.8 \times 10^{-16} \mathrm{~cm}^{2}$ & 16 \\
\hline$\sigma_{\mathrm{T}_{1} \mathrm{~T}_{2}}$ & $\begin{array}{l}\text { Triplet absorption cross section } \\
\left(\mathrm{T}_{1}+\hbar \omega \rightarrow \mathrm{T}_{2}^{\prime}\right)\end{array}$ & $(1.1 \pm 0.1) \times 10^{-16} \mathrm{~cm}^{2}$ & This work \\
\hline$\sigma_{\mathrm{T}_{1} \mathrm{~T}_{4}}$ & $\begin{array}{l}\text { Triplet absorption cross section at } 532 \mathrm{~nm} \\
\left(\mathrm{~T}_{1}+\hbar \omega \rightarrow \mathrm{T}_{4}\right)\end{array}$ & $7.4 \times 10^{-17} \mathrm{~cm}^{2}$ & 16 \\
\hline$\tau_{\mathrm{S}_{1}}$ & $\mathrm{~S}_{1}$ lifetime & $89 \mathrm{ps}$ & $16-18$ \\
\hline$\tau_{\mathrm{T}_{1}}$ & $\begin{array}{l}\text { Lifetime of } \mathrm{T}_{1} \text { (includes both phosphorescence and } \\
\text { oxygen quenching) }\end{array}$ & $3 \mu \mathrm{s}$ & 19 \\
\hline$\tau_{\mathrm{T}_{4}}$ & Lifetime of $\mathrm{T}_{4}$ & $50 \mathrm{fs}$ & 16 \\
\hline$\tau_{\mathrm{T}_{n}}$ & Lifetime of $T_{n}$ & fitting parameter & \\
\hline$\Phi_{\text {isc }}$ & Intersystem crossing yield $\left(\mathrm{S}_{1} \rightarrow \mathrm{T}_{1}\right)$ & 0.98 & 18,19 \\
\hline$\Phi_{\text {risc }, T_{4}}$ & Reverse intersystem crossing yield $\left(\mathrm{T}_{4} \rightarrow \mathrm{S}_{1}^{\prime}\right)$ & 0.8 & 13 \\
\hline$\Phi_{\text {risc }, T_{n}}$ & $\begin{array}{l}\text { Reverse intersystem crossing yield } \\
\left(\mathrm{T}_{n} \rightarrow \mathrm{S}_{1}^{\prime}\right)\end{array}$ & fitting parameter & \\
\hline$F_{1}$ & First pump-pulse fluence $(\lambda=532 \mathrm{~nm})$ & $(8.8 \pm 0.5) \times 10^{15}$ photons $/ \mathrm{cm}^{2}$ & \\
\hline$F_{2}$ & Second pump-pulse fluence $(\lambda=1064 \mathrm{~nm})$ & varied & \\
\hline$k_{\mathrm{ic}, \mathrm{T}_{4}}$ & Internal conversion rate $\left(\mathrm{T}_{4} \rightarrow \mathrm{T}_{1}\right)$ & $\left(1-\Phi_{\text {risc, } \mathrm{T}_{4}}\right) / \tau_{\mathrm{T}_{4}}$ & \\
\hline$k_{\mathrm{ic}, \mathrm{T}_{n}}$ & Internal conversion rate $\left(\mathrm{T}_{n} \rightarrow \mathrm{T}_{1}\right)$ & $\left(1-\Phi_{\text {risc, } \mathrm{T}_{n}}\right) / \tau_{\mathrm{T}_{n}}$ & \\
\hline$k_{\text {isc }}$ & Intersystem crossing rate & $\Phi_{\mathrm{isc}} / \tau_{\mathrm{S}_{\mathrm{I}}}$ & \\
\hline$k_{r, \mathrm{~T}_{n}}$ & Thermalization rate $\left(\mathrm{T}_{n}^{\prime} \rightarrow \mathrm{T}_{n}\right)$ & fitting parameter & \\
\hline$k_{r}$ & Thermalization rate $\left(S_{1}^{\prime} \rightarrow S_{1}\right)$ & $10^{12} s^{-1}$ & 16 \\
\hline$k_{\text {risc, } \mathrm{T}_{4}}$ & $\begin{array}{l}\text { Reverse intersystem crossing rate } \\
\left(\mathrm{T}_{4} \rightarrow \mathrm{S}_{1}^{\prime}\right)\end{array}$ & $\Phi_{\text {risc }, T_{4}} / \tau_{\mathrm{T}_{4}}$ & \\
\hline$k_{\text {risc, } \mathrm{T}_{n}}$ & $\begin{array}{l}\text { Reverse intersystem crossing rate } \\
\left(\mathrm{T}_{n} \rightarrow \mathrm{S}_{1}^{\prime}\right)\end{array}$ & $\overline{\Phi_{\text {risc }, T_{n}} / \tau_{\mathrm{T}_{n}}}$ & \\
\hline$k_{\mathrm{T}_{1}}$ & $\mathrm{~T}_{1}$ relaxation rate & $1 / \tau_{\mathrm{T}_{1}}$ & \\
\hline
\end{tabular}


(L)

$$
\cdot p p(\mathfrak{l}){ }^{\mathrm{I}} \mathrm{S}_{d} \int_{\infty+\downarrow}^{0} \frac{{ }^{\mathrm{I}} \mathrm{S}_{\mathfrak{z}}}{{ }^{f}{ }_{\Phi}}=\tau_{f}
$$

pue

(9)

$$
t p(t)^{\mathrm{I}} \mathrm{S}_{d} \int_{0}^{\infty-{ }^{\mathrm{I}} \mathrm{S}_{2}} \frac{I f}{{ }^{f} \boldsymbol{\Phi}}={ }^{\prime}
$$

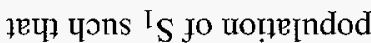

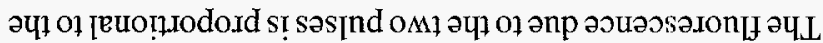

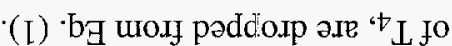

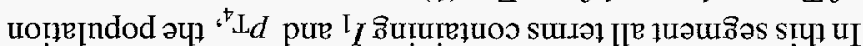

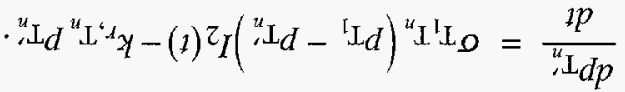

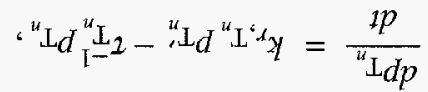

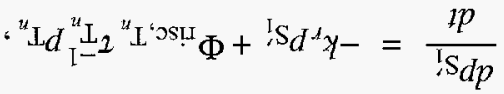

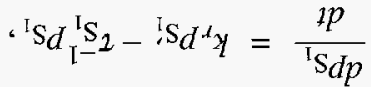

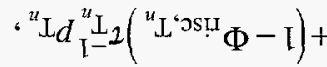

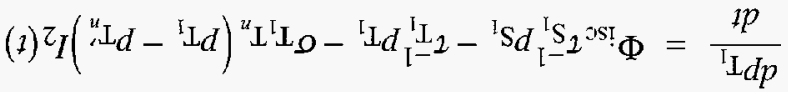

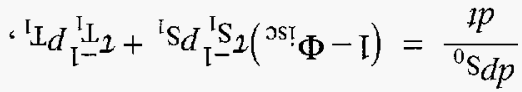

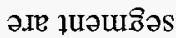

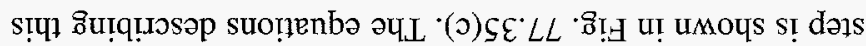

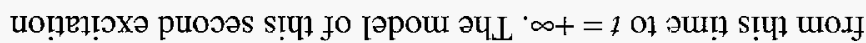

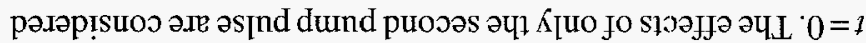

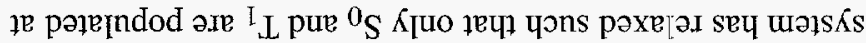

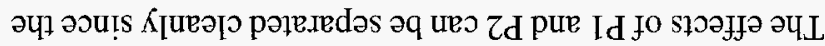

'əs[nd dund

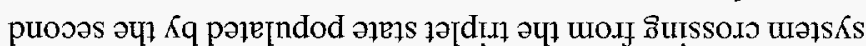

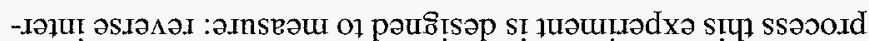

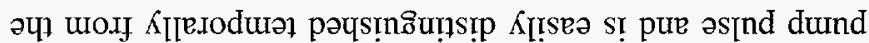

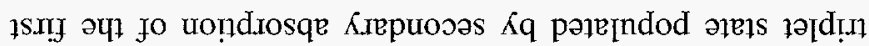

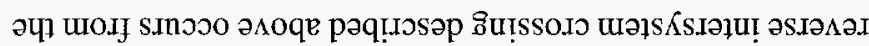

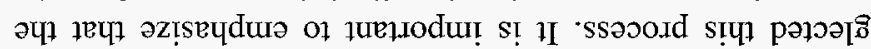

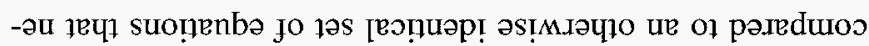

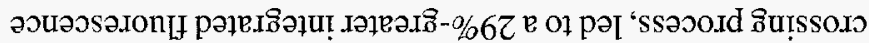

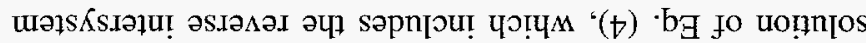

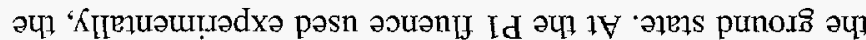

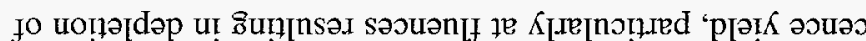

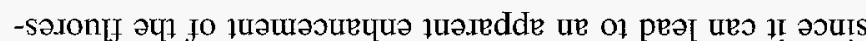

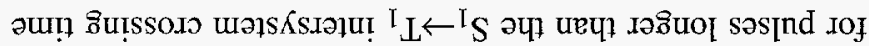

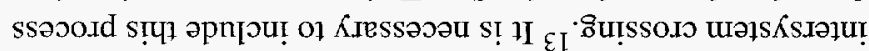

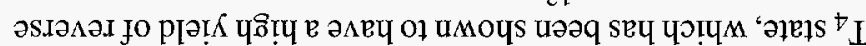
ә

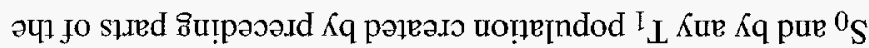

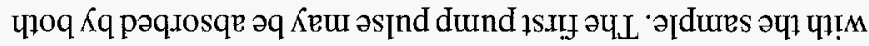

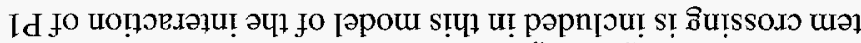

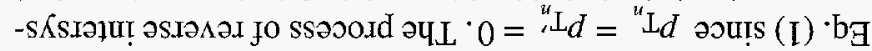

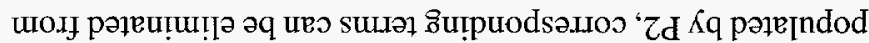

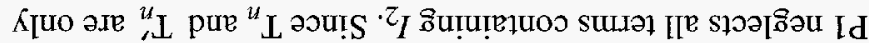

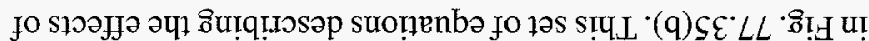

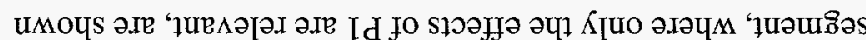

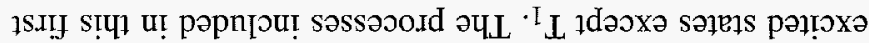

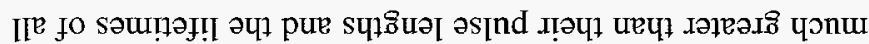

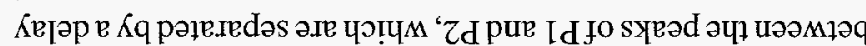

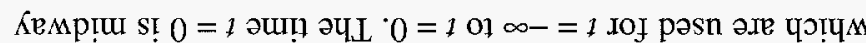

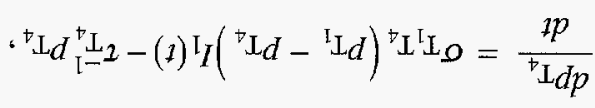

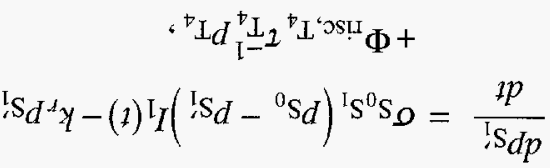

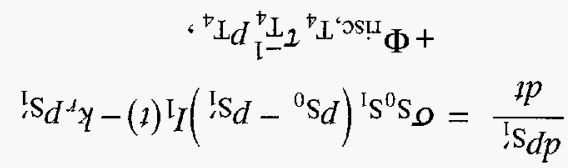

$$
\begin{aligned}
& { }^{\mathrm{I}} \mathrm{S}_{d}{ }_{I^{-} \mathrm{S}_{2}}^{\mathrm{I}}{ }^{\mathrm{I}} \mathrm{S}_{d}{ }^{\prime}{ }_{y}=\frac{t p}{{ }^{\mathrm{T}} \mathrm{S}_{d p}}
\end{aligned}
$$

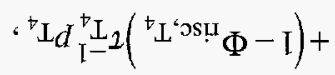

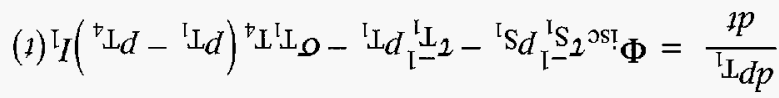$$
\text { ، } I_{d}{ }_{1}^{\mathrm{I}} \mathrm{L}_{1}+
$$

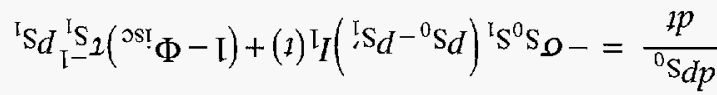


where $\Phi_{f}$ is the fluorescence yield. The two-step, laser-induced fluorescence ratio $f_{R}$ is defined by

$$
f_{R} \equiv \frac{f_{2}}{f_{1}}
$$

This is a convenient quantity to compare with experimental results since fluorescence yield, collection, and detection efficiency factors are eliminated.

The $\mathrm{T}_{n}$ photophysical parameters are determined by fitting this model of the two-step, laser-induced fluorescence process to the fluence-dependent $f_{R}$ obtained experimentally. As will be discussed later, extraction of the parameters requires $f_{R}$ measurements over a range of $\mathrm{P} 2$ fluences, which, at the upper limit, are sufficient to partially deplete the lowest triplet state. In addition, the length of the second pump pulse must exceed the lifetime of $\mathrm{T}_{n}$. A numerical approach is required since under these conditions analytical solutions cannot be obtained easily. The numerical analysis consists of three major components: (a) a calculation of the fluence-dependent $f_{R}$ for a given set of $\mathrm{T}_{n}$ photophysical parameters, (b) an algorithm that optimizes these parameters to provide the best fit to the experimental data, and (c) an estimate of the precision to which the extracted parameters are known based on a randomization and re-optimization technique.

Calculation of the fluence-dependent, two-step laserinduced fluorescence ratio was based on the sequential solution of the rate equations given in Eqs. (4) and (5). These rate equations were solved using Runge-Kutta numerical integration. The agreement between the $f_{R}$ obtained from this model and the experimental data can be quantified by the $\chi^{2}$ statistic, which is summed over the set $F_{1}, F_{2}$ for which experimental measurements of the TSLIF ratio, $f_{R \text {,expt }}$, were made. The standard deviations of those measurements are given by $\sigma_{R \text {,expt }}$. The next step is to search parameter space in order to find the values of $k_{r, \mathrm{~T}_{n}}, \tau_{\mathrm{T}_{n}}$, and $\Phi_{\text {risc, } \mathrm{T}_{n}}$ that minimize $\chi^{2}$. The optimization algorithm used is the downhill simplex method. ${ }^{20}$

The downhill simplex method will find the set of parameters that minimizes $\chi^{2}$, but it does not report the precision with which these parameters are known given the uncertainties in the experimental measurements. This precision was estimated by running the optimization routine on sets of TSLIF ratio measurements, $f_{R, \text { mix }}$, calculated from

$$
f_{R, \text { mix }}\left(F_{1}, F_{2}\right)=f_{R, \text { expt }}\left(F_{1}, F_{2}\right)+r \sigma_{R, \text { expt }}\left(F_{1}, F_{2}\right),
$$

where $r$ is a uniformly distributed random number between -1 and 1 . The standard deviations of the parameters found in minimizing ten such data sets provide the estimated precision to which the parameters are known.

An analytical model of two-step laser-induced fluorescence that is limited to low-intensity and low-fluence conditions can be developed. In this regime the fluorescence signals are given by

$$
f_{1}=a \sigma_{\mathrm{S}_{0} \mathrm{~S}_{1}} F_{1}
$$

and

$$
f_{2}=a \Phi_{\mathrm{isc}} \Phi_{\mathrm{risc}, \mathrm{T}_{n}} \sigma_{\mathrm{S}_{0} \mathrm{~S}_{1}} F_{1} \sigma_{\mathrm{T}_{1} \mathrm{~T}_{n}} F_{2},
$$

where $a$ includes fluorescence yield, collection, and detection factors. Calculating the two-step laser-induced fluorescence ratio from Eqs. (10) and (11) gives

$$
f_{R}=\Phi_{\text {isc }} \Phi_{\text {risc, } \mathrm{T}_{n}} \sigma_{\mathrm{T}_{1} \mathrm{~T}_{n}} F_{2}
$$

Although this expression cannot be used to determine $\tau_{\mathrm{T}_{n}}$ and $k_{r, T_{n}}$, it is useful for estimating upper limits on $\Phi_{\text {risc, } T_{n}}$ when there is an undetectable two-step laser-induced fluorescence signal.

\section{Results}

The triplet-triplet absorption spectrum of rose bengal in the near infrared (Fig. 77.36) is derived from a series of transient absorption measurements acquired by laser flash photolysis. There is no measurable ground-state absorption in this region. Detector insensitivity prevented the extension of this spectrum beyond $1100 \mathrm{~nm}$. Using the intensity variation method ${ }^{21}$ it was found that the absorption has a peak between 1050 and $1075 \mathrm{~nm}$ with a cross section $\sigma_{\mathrm{T}_{1} \mathrm{~T}_{2}}=(1.1 \pm 0.1) \times 10^{-16} \mathrm{~cm}^{2}$.

Figure 77.37 shows two-step laser-induced fluorescence results for $\mathrm{T}_{2}$. The ratio of two-step to one-step fluorescence $f_{R \text {,expt }}$ versus the fluence of the second pump pulse $\left(\lambda_{2}=\right.$ $1064 \mathrm{~nm})$ is plotted. The first pump-pulse fluence $\left(\lambda_{1}=\right.$ $532 \mathrm{~nm}$ ) was held approximately constant at $3.3 \pm 0.2 \mathrm{~mJ} / \mathrm{cm}^{2}$, and the resulting one-step fluorescence varied by less than $2 \%$. No emission following P2 was detected when P1 was blocked. Each point in this plot represents the average of from 26 to 370 double-pulse excitations. The error bars indicate the corresponding standard deviations. In addition, the spectrum of the 532-nm + 1064-nm excited emission was measured and found to be the same as the $S_{1} \rightarrow S_{0}$ fluorescence spectrum, 


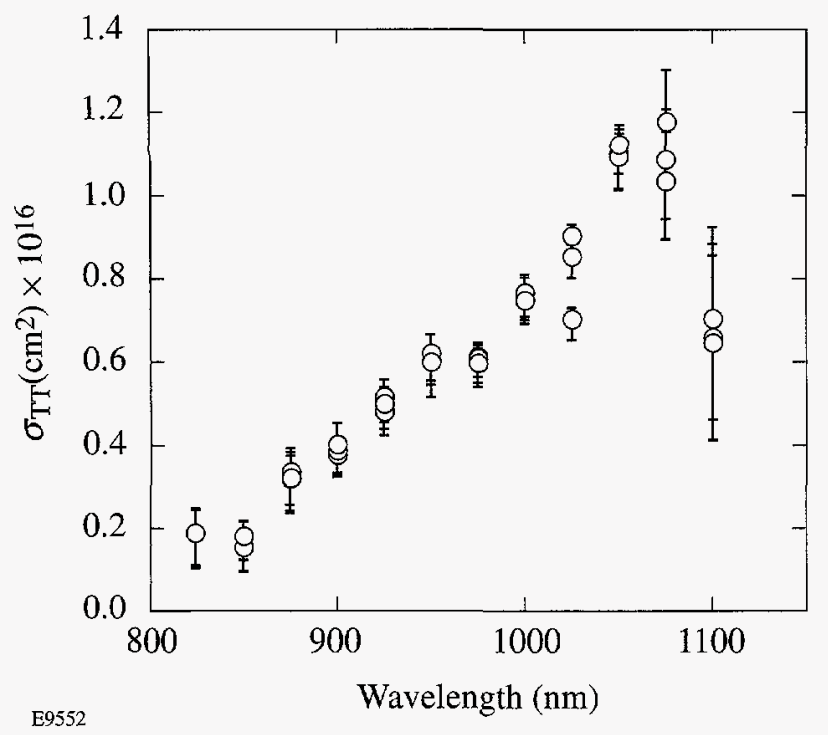

Figure 77.36

Triplet-triplet absorption spectrum of rose bengal in the near infrared.

confirming that the TSLIF results from repopulation of $\mathrm{S}_{1}$. The parameters $\Phi_{\text {risc, } \mathrm{T}_{2}}, \tau_{\mathrm{T}_{2}}$, and $k_{r, \mathrm{~T}_{2}}$ can be determined by analyzing the nonlinear dependence of $f_{R}$ on $F_{2}$ using the multistate kinetic model described in the Analysis section. This analysis of the data shown in Fig. 77.37 gives $\Phi_{\text {risc, } \mathrm{T}_{2}}=$ $0.0142 \pm 0.0003, \tau_{\mathrm{T}_{2}}=5.8 \pm 1.6 \mathrm{ps}$, and $k_{r, \mathrm{~T}_{2}}=1.30 \pm 0.18 \mathrm{ps}^{-1}$ with $\chi^{2}=0.011$

Similar measurements probing $\mathrm{T}_{3}\left(\lambda_{1}=532 \mathrm{~nm}, \lambda_{2}=\right.$ $632 \mathrm{~nm}$ ) failed to detect any two-step laser-induced fluorescence. Based on the fluorescence detection limits, the quantum yield of reverse intersystem crossing from $\mathrm{T}_{3}$ can be constrained to $\Phi_{\text {risc, } \mathrm{T}_{3}}<0.06$ using Eq. (12) with $\sigma_{\mathrm{T}_{1} \mathrm{~T}_{3}}$ determined from Ref. 22 and $f_{R}$ set equal to the uncertainty in the TSLIF measurement.

\section{Discussion}

Although no analytical expression can be given for $f_{R}\left(F_{1}, F_{2}\right)$ that is applicable for the high fluences used in these experiments, it is possible to explain qualitatively the shape of the $f_{R}$ versus $F_{2}$ curve shown in Fig. 77.37. This explanation also provides some justification for why the kinetic model analysis is sensitive to the lifetime and thermalization rate of the upper triplet state. Under low-fluence and low-intensity conditions, Eq. (12) predicts that $f_{R}$ will increase linearly with $F_{2}$. Deviations from the predicted linear response are expected to occur for P2 with sufficiently high intensity or fluence. Under conditions where the pulse length is shorter than the lifetime of $\mathrm{T}_{2}$, the saturation fluence $F_{\text {sat }}=\left(\sigma_{\mathrm{T}_{1} \mathrm{~T}_{2}}\right)^{-1}$ for

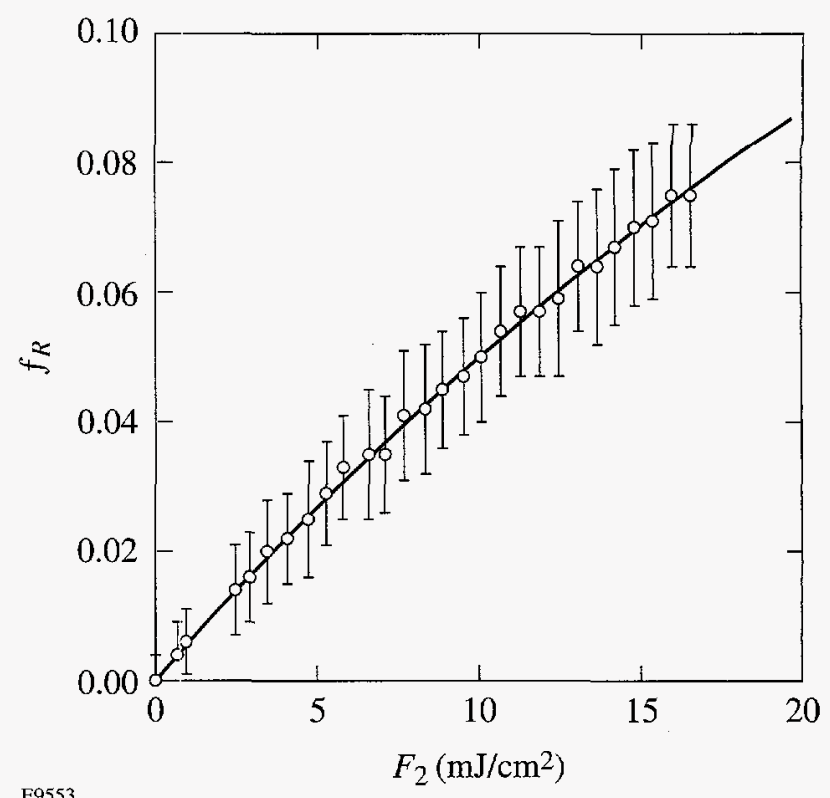

E9553

Figure 77.37

Fluence dependence of two-step laser-induced fluorescence ratio $f_{R}$. Delay between excitation pulses: 34 ns. Circles are averages of from 26 to 370 double-pulse excitations with error bars indicating the standard deviations. The curve represents the best fit $\left(\chi^{2}=0.011\right)$ calculated from solutions of Eqs. (4) and (5) using the parameter values given in Table 77.III. The photophysical parameters determined from the fitting procedure are $\Phi_{\text {risc, } \mathrm{T}_{2}}=0.0142 \pm 0.0003, \tau_{\mathrm{T}_{2}}=5.8 \pm 1.6 \mathrm{ps}$, and $k_{r, \mathrm{~T}_{2}}=1.30 \pm 0.18 \mathrm{ps}^{-1}$.

$\mathrm{T}_{1} \rightarrow \mathrm{T}_{2}$ excitation is $9 \times 10^{15}$ photons $/ \mathrm{cm}^{2}\left(1.7 \mathrm{~mJ} / \mathrm{cm}^{2}\right)$. Multiple excitations are possible, however, for pulses that are longer than the lifetime of $\mathrm{T}_{2}$. This allows the two-step laserinduced fluorescence ratio to continue to grow beyond the short-pulse saturation fluence limit. Limits on the growth of the two-step laser-induced fluorescence are not solely fluence dependent. The maximum rate at which population can be excited to the upper triplet state is limited by the thermalization rate $k_{r, T_{2}}$. In addition, the maximum number of excitation cycles that can be achieved during a pulse is limited by the upper triplet lifetime $\tau_{\mathrm{T}_{2}}$ and the length of the second pump pulse $\delta_{2}$. Since the nonlinear portion of the $f_{R}$ curve is dependent on the upper triplet lifetime and its thermalization rate, it is possible to extract these parameters from a fit of the kinetic model to data obtained under high-intensity and highfluence conditions where the deviation from linearity becomes significant.

The multistate kinetic model described in Eq. (1) and Fig. 77.35 is not the only possible explanation for fluorescence following 532-nm + 1064-nm excitation. An alternative 
model that deserves consideration includes absorption of 1064-nm light by $T_{2}$ to populate $T_{4}$, a state already known to have a high reverse intersystem crossing yield..$^{13}$ On the basis of energetic considerations, the $\mathrm{T}_{2} \rightarrow \mathrm{T}_{4}$ absorption process appears to be plausible, although restrictions such as those based on parity may disallow this transition. If reverse intersystem crossing were to occur predominantly from $\mathrm{T}_{4}$, then the expression for $f_{R}$ given in Eq. (12) should be modified to give

$$
f_{R}=\Phi_{\text {isc }} \Phi_{\text {risc, } \mathrm{T}_{4}} \sigma_{\mathrm{T}_{1} \mathrm{~T}_{2}} \sigma_{\mathrm{T}_{2} \mathrm{~T}_{4}} F_{2}^{2}
$$

According to this model, $f_{R}$ increases quadratically rather than linearly in $F_{2}$ since population of $\mathrm{T}_{4}$ from $\mathrm{T}_{1}$ requires the sequential absorption of two 1064-nm photons. In addition, $f_{R}$ is expected to saturate at a value greater than $\Phi_{\text {isc }} \Phi_{\text {risc, } \mathrm{T}_{4}}=0.78$. The experimental data shown in Fig. 77.37 do not exhibit this behavior, which justifies our elimination of this alternative model.

No two-step laser-induced fluorescence was detected in the $532-\mathrm{nm}+632-\mathrm{nm}$ experiment. This experiment was performed under conditions much less favorable than for the 532-nm + 1064-nm experiment. Both the maximum P2 fluence and the triplet-triplet absorption cross section were significantly less at $632 \mathrm{~nm}$ compared to $1064 \mathrm{~nm}$. Even with these limitations, however, the value of $\Phi_{\text {risc, } T_{3}}$ can be determined to be less than 0.06 . This result disagrees with a yield of 0.72 for this state reported previously. ${ }^{15}$

Ketsle et al. attempted to measure the yield of reverse intersystem crossing through measurements of the change in $\mathrm{T}_{1}$ absorption (and therefore, concentration) in a two-step excitation experiment. ${ }^{15}$ Immediately following P2, a decrease in the concentration $\Delta C_{a b}$ of $T_{1}$ was observed, which was followed by a partial recovery $\Delta C_{a c}$, as shown in Fig. 77.38, which is a sketch showing the key features in the transient signal plotted in Fig. I of Ref. 15. The points $a, b$, and $c$ in Fig. 77.38 represent the times immediately prior to $\mathrm{P} 2$, immediately following $\mathrm{P} 2$, and after the fast recovery of the transient bleaching, respectively. The lack of complete recovery is due to reverse intersystem crossing from the higher-lying triplet populated by $\mathrm{P} 2$. From these concentration changes Ketsle $e t a l$. calculated the reverse intersystem crossing yield using the formula

$$
\frac{\Delta C_{a c}}{\Delta C_{a b}}=\Phi_{\text {risc }, T_{3}}
$$

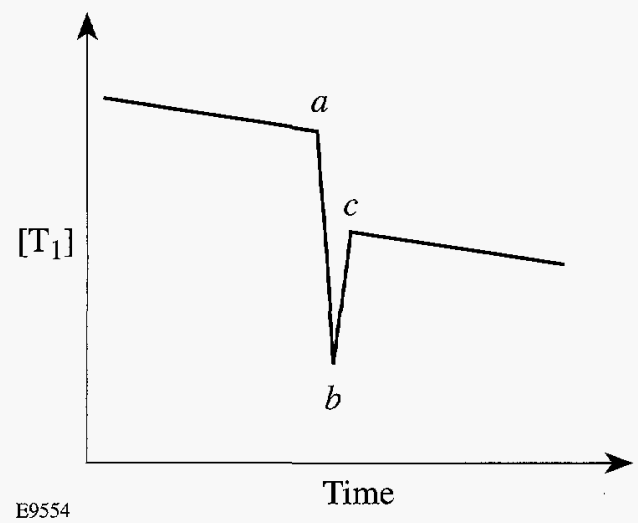

Figure 77.38

Schematic of transient changes in $T_{1}$ concentration (based on Fig. $I$ in Ref. 15). The points $a, b$, and $c$ in the figure represent the times immediately prior to $\mathrm{P} 2$, immediately following $\mathrm{P} 2$, and after the fast recovery of the transient bleaching, respectively

Equation (14) attributes the bleaching to the entire fraction undergoing reverse intersystem crossing. It is more appropriate, however, to interpret bleaching as due to the fraction that undergoes reverse intersystem crossing and in addition does not repopulate the triplet manifold through $S_{1} \rightarrow T_{1}$ transfer, implying

$$
\frac{\Delta C_{a c}}{\Delta C_{a b}}=\Phi_{\text {risc }, \mathrm{T}_{3}}\left(1-\Phi_{\mathrm{isc}}\right)
$$

Indeed, this latter interpretation of the bleaching fraction agrees with that used by Redmond et al. ${ }^{12}$ Recalculating a yield based on Eq. (15) using the Ketsle et al. bleaching fraction data found in Table I of Ref. 15 gives $\Phi_{\text {risc, } T_{3}}>1$. Since this quantum yield cannot exceed unity, it appears that their experimental data was obtained under conditions in which the assumptions used to derive these equations do not apply. In particular, these equations are valid only under conditions in which $\Delta C_{a b}$ is proportional to the number of photons absorbed by $T_{1}$. This can occur only when the length of the exciting pulse is shorter than the lifetime of the upper triplet state $\left(\delta_{2}<<\tau_{\mathrm{T}_{3}}\right)$ and when the transient absorption detection system is capable of responding on this same time scale. Ketsle et al. do not report the length of their second pump pulse but state only that it is from a ruby laser. It appears likely that their excitation pulse is longer than several nanoseconds, which is much greater than the expected upper triplet lifetime of picoseconds or less. In addition, the time response of their transient absorption detection system is not reported. The use of long pulses or slow detection systems with this transient 
absorption technique will lead to an underestimate of the number of absorbed photons, thus leading to values of $\Phi_{\text {risc, }} T_{3}$ that exceed unity. The equipment requirements are not as demanding for fluorescence methods of measuring reverse intersystem crossing.

Reverse intersystem crossing yields have been calculated for a growing number of molecules. An aspect of this study that makes it of particular interest is that these yields have now been measured for several triplet states of rose bengal. Previous workers in this field have suggested that population excited to $\mathrm{T}_{n}$ relaxes rapidly to the next-lowest triplet state, and that the triplet-singlet transfer is predominantly due to reverse intersystem crossing from this less-energetic state. ${ }^{13,23}$ According to this model, the reverse intersystem crossing yield should be independent of the high-lying triplet state initially excited; experimental measurements clearly contradict this prediction with high yields of $\Phi_{\text {risc, } T_{4}}=0.80$ if $\mathrm{T}_{4}$ is initially excited, ${ }^{13}$ to much lower yields of $\Phi_{\text {risc, } T_{2}}=0.0142$ for the case of direct population of $T_{2}$ (measured in this work).

To understand these results it is instructive to consider the energies of the relevant triplet and singlet states. The energies of the singlet states can be estimated from the peaks of the ground-state absorption spectrum. Similarly, the energies of the triplet states relative to $T_{1}$ can be estimated from triplettriplet absorption spectra (the present work and Refs. 8 and 24). The energy of $T_{1}$ in methanol is $1.75 \mathrm{eV}^{25}$ These results have been compiled in Table 77.IV. The energy gaps between the excited triplet states and the nearest less-energetic singlet state are $\Delta E\left(\mathrm{~T}_{2}-\mathrm{S}_{2}\right)=0.51 \mathrm{eV}, \Delta E\left(\mathrm{~T}_{3}-\mathrm{S}_{3}\right)=0.35 \mathrm{eV}$, and $\Delta E\left(\mathrm{~T}_{4}-\mathrm{S}_{4}\right)=0.08 \mathrm{eV}$. Thus we find that the transition with the smallest energy gap exhibits the greatest reverse intersystem crossing yield $\left(\Phi_{\text {risc }, T_{2}}=0.0142, \Phi_{\text {risc, } \mathrm{T}_{3}}<0.06\right.$, $\Phi_{\text {risc, } \mathrm{T}_{4}}=0.80$ ). Although this ordering is consistent with a simple interpretation of the energy gap law for nonradiative transitions, which states that reverse intersystem crossing is likely to be most favorable when there is a small energy gap between the triplet state and a nearby singlet, such an interpretation must be considered critically. As developed by Englman and Jortner, ${ }^{26}$ the energy gap law applies to a particular tripletsinglet pair, whereas here we are considering three such pairs. The strength of the spin-orbit coupling between different states may vary by several orders of magnitude. Since we do not know the values of the coupling parameters for the three transitions under consideration, it is impossible to definitively attribute the entire variation in reverse intersystem crossing yield to differences in the energy gap.
Table 77.IV: Energies of rose bengal excited states. Singletstate energies are estimated from the ground-state absorption spectrum. Triplet-state energies are estimated from the $\mathrm{T}_{1}$ absorption spectrum.

\begin{tabular}{|c|c|c|}
\hline \hline State & Energy (eV) & Ref. \\
\hline $\mathrm{S}_{1}$ & 2.10 & This work \\
\hline $\mathrm{S}_{2}$ & 2.41 & This work \\
\hline $\mathrm{S}_{3}$ & 3.51 & This work \\
\hline $\mathrm{S}_{4}$ & 3.95 & This work \\
\hline $\mathrm{T}_{1}$ & 1.75 & 25 \\
\hline $\mathrm{T}_{2}$ & 2.92 & This work \\
\hline $\mathrm{T}_{3}$ & 3.86 & 8 \\
\hline $\mathrm{T}_{4}$ & 4.03 & 24 \\
\hline \hline
\end{tabular}

\section{Conclusion}

We have presented what we believe to be the first study of a triplet state of rose bengal that is produced by $1064-\mathrm{nm}$ excitation of $\tau_{1}$. The triplet-triplet absorption cross section was measured between $825 \mathrm{~nm}$ and $1100 \mathrm{~nm}$. This state was further characterized using two-step laser-induced fluorescence to determine its thermalization rate, lifetime, and quantum yield of reverse intersystem crossing. Similar two-step laser-induced fluorescence measurements were made of the triplet excited by 632-nm light.

In earlier work, the reverse intersystem crossing yield was predicted to be independent of which higher-lying triplet state was initially excited. The present work finds that the yields for triplets excited by red and near-infrared light, $\mathrm{T}_{3}$ and $\mathrm{T}_{2}$, are much less than those reported earlier for the more-energetic state $T_{4}$, which is populated by green light. ${ }^{13}$ An analysis of the triplet-triplet absorption spectrum and the ground-state absorption spectrum shows that $T_{4}$ is energetically close to a state in the singlet manifold, whereas the corresponding gaps are significantly greater for $T_{2}$ and $T_{3}$.

\section{ACKNOWLEDGMENT}

This work has been supported by the U.S. Department of Energy Office of Inertial Confinement Fusion under Cooperative Agreement No. DEFC03-92SF1946C, the University of Rochester, the New York State Energy Research and Development Authority, and by U.S. Public Health Service grant CA68409. The support of DOE does not constitute an endorsement by $\mathrm{DOE}$ of the views expressed in this article. 


\section{REFERENCES}

1. O. Raab, Infusoria Z. Biol. 39, 524 (1900).

2. V. H. Tappeiner and A. Jesionek, Muench. Med. Wochneshr. 47, 2042 (1903).

3. F. H. J. Figge, G. S. Weiland, and L. O. J. Manganiello, Proc. Soc. Exp. Biol. Med. 68, 640 (1948).

4. R. L. Lipson, E. J. Baldes, and A. M. Olsen, J. Natl. Cancer Inst. 26, 1 (1961).

5. T. J. Dougherty, J. Natl. Cancer Inst. 52, 1333 (1974).

6. H. I. Pass, J. Natl. Cancer Inst. 85, 443 (1993).

7. T. J. Dougherty et al., J. Natl. Cancer Inst. 90, 889 (1998).

8. G. Smith et al., Photochem. Photobiol. 59, 135 (1994).

9. S. P. McGlynn, T. Azumi, and M. Kinoshita, Molecular Spectroscopy of the Triplet State (Prentice-Hall, Englewood Cliffs, NJ, 1969).

10. S. Kobayashi, K. Kikuchi, and H. Kokubun, Chem. Phys. Lett. 42, 494 (1976); S. Kobayashi, K. Kikuchi, and H. Kokubun, Chem. Phys. 27, 399 (1978).

11. W. G. McGimpsey and J. C. Scaiano, J. Am. Chem. Soc. 111, 335 (1989).

12. R. W. Redmond et al., J. Phys. Chem. A 101, 2773 (1997).
13. S. Reindl and A. Penzkofer, Chem. Phys. 211, 431 (1996).

14. N. Durán and G. Cilento, Photochem. Photobiol. 32, 113 (1980).

15. G. A. Ketsle, L. V. Levshin, and S. N. Letuta, Opt. Spectrosc. (USSR) 68, $202(1990)$.

16. H. Stiel et al., J. Photochem. Photobiol. B: Biol. 33, 245 (1996).

17. G. R. Fleming et al., J. Am. Chem. Soc. 99, 4306 (1977).

18. M. A. J. Rodgers, Chem. Phys. Lett. 78, 509 (1981).

19. P. C. C. Lee and M. A. J. Rodgers, Photochem. Photobiol. 45, 79 (1987).

20. W. H. Press et al., Numerical Recipes in FORTRAN: The Art of Scientific Computing, 2nd ed. (Cambridge University Press, Cambridge, England, 1992).

21. I. Carmichael and G. L. Hug, J. Phys. Chem. Ref. Data 15, 1 (1986).

22. P. Murasecco-Suardi et al., Helv. Chim. Acta. 70, 1760 (1987).

23. H. Fukumura et al., J. Photochem. Photobiol. A: Chem. 42, 283 (1988).

24. C. R. Lambert et al., Photochem. Photobiol. 63, 154 (1996).

25. T. Shen et al., J. Photochem. Photobiol. A: Chem. 47, 203 (1989).

26. R. Englman and J. Jortner, Mol. Phys. 18, 145 (1970). 


\section{Picosecond Response of Optically Driven Y-Ba-Cu-O Microbridge and Josephson-Junction Integrated Structures}

Photoexcitation studies of superconductors have been a subject of intense investigation for the last 20 years. Early experiments were performed on metallic superconductors using nanosecond and picosecond pulses and were concentrated on the dynamics of the photon-induced, superconducting-tonormal transition. ${ }^{1,2}$ The discovery of high-temperature superconductors (HTS) prompted a new series of transient photoexcitation experiments. Experiments with optical-pulsedriven current-biased samples and direct measurements of the resulting voltage transient provide the most direct information on nonequilibrium processes in HTS. These experiments are also most relevant when evaluating the potential of HTS materials for fast photodetector applications. Recently, we have observed the single-picosecond electrical response of a current-biased $\mathrm{YBa}_{2} \mathrm{Cu}_{3} \mathrm{O}_{7-x}$ (YBCO) microbridge exposed to femtosecond optical pulses. ${ }^{3-6}$ The experiments were conducted in the temperature range from $4.2 \mathrm{~K}$ to $80 \mathrm{~K}$, using our subpicosecond electro-optic (EO) sampling system. ${ }^{4}$ The two mechanisms responsible for the picosecond response of the YBCO microbridge have been identified: nonequilibrium kinetic inductance and hot-electron heating. ${ }^{7}$ The corresponding electrical transient was either a 2-ps-wide oscillation for the kinetic inductance response, or a single-picosecond spike for electron heating in the resistive state. ${ }^{6}$

Picosecond-impulse excitation of Josephson junctions has been extensively studied theoretically. ${ }^{8-10}$ The simulations showed that a junction response is delayed with respect to the excitation impulse by a turn-on delay time $\tau_{D}$, which depends on both the junction bias and the critical current $I_{c}$ overdrive $\left(I_{c}\right.$ is defined as the maximum superconducting current that can flow through a Josephson junction). The rise time $\tau_{R}$ of the junction switching transient was also calculated and found to depend on the amount of the $I_{c}$ overdrive, as well as on the product of the junction's normal resistance $x$ the junction's capacitance $\left(R_{N} C_{J}\right)$. In the case of the single-flux-quantum (SFQ) pulse generation by resistively shunted junctions (RSJ), the generated pulse has been predicted to have an amplitude equal to $2 I_{c} R_{N}$ and a width corresponding to $\Phi_{0} / 2 I_{c} R_{N},{ }^{11}$ where $\Phi_{0}=2.07 \mathrm{mV} \cdot \mathrm{ps}$.
In this article, we report our studies on the picosecond photoresponse of a current-biased YBCO microbridge coupled to a bicrystal YBCO Josephson junction. Femtosecond optical pulses were used to excite the microbridge in the resistive state $^{5}$ and to generate a series of picosecond-duration electrical transient pulses. These transients were in turn applied to switch the grain-boundary Josephson junction. The junction response was superimposed on the large feedthrough signal, but the junction signal could be identified due to its dependence on the bias current.

\section{Sample Fabrication and Experimental Setup}

The test structures, consisting of coplanar strip (CPS) transmission lines, were fabricated on (100) MgO bicrystal substrates, using a standard laser ablation technique and ionbeam etching. ${ }^{12}$ YBCO films 50 to $100 \mathrm{~nm}$ thick were deposited at the substrate temperature $800^{\circ} \mathrm{C}$ and at the ambient oxygen pressure of $0.35 \mathrm{mbar}$. The deposition was followed by an annealing cycle in pure oxygen. Next, a 50-nm-thick gold layer was sputtered in situ on top of YBCO thin film at room temperature at an argon pressure of $0.05 \mathrm{mbar}$. The test structures were prepared in a two-step process. First, they were photolithographically defined and then etched with a lowcurrent-density $\left(1 \mathrm{~mA} / \mathrm{cm}^{2}\right)$ ion argon beam. In the second step, the Au layer was removed from the top of the junction and bridge areas, using the same low-intensity ion etching. As a result, eight 8-mm-long CPS lines, containing $10-\mu \mathrm{m} \times 5-\mu \mathrm{m}$ bridges and 5 - $\mu \mathrm{m}$-wide bicrystal Josephson junctions, were fabricated on each substrate. The 8-mm length of CPS was chosen to restrict the end-of-line reflections, assuring a 50-pslong reflection-free measurement time-window. The schematic of our CPS line and the measurement configuration are shown in Fig. 77.39. We note that the test structure is not a Josephson-junction transmission line, ${ }^{11}$ but rather a high characteristic impedance $(80 \Omega)$ CPS line with the junction electrodes representing high inductance. This type of experimental configuration was chosen for practical fabrication considerations and will be improved in future designs. 


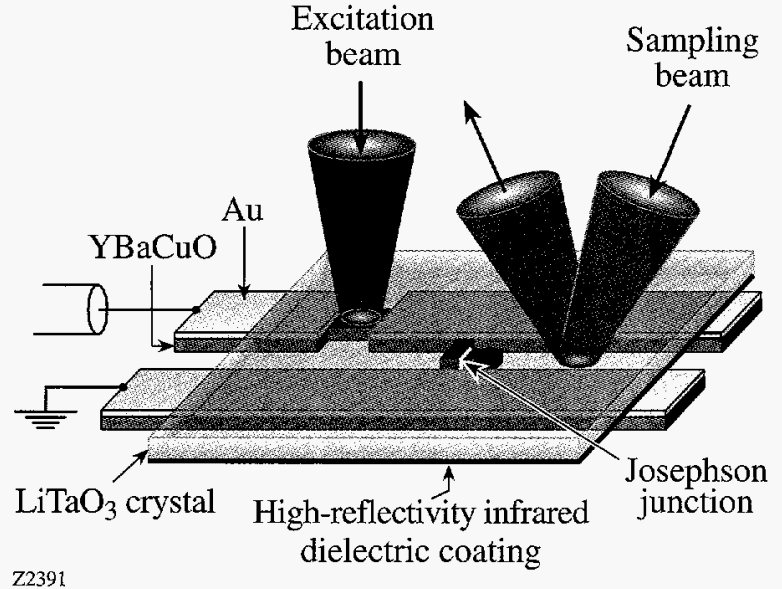

Figure 77.39

Experimental setup of CPS line and measurement configuration.

Our optical system for the femtosecond pulse excitation and EO sampling detection is described in detail in Ref. 4. Briefly, a mode-locked Ti:sapphire laser, operating at a repetition rate of $76 \mathrm{MHz}$, was used to generate $100-\mathrm{fs}$ pulses at a wavelength of $800 \mathrm{~nm}$. To perform EO characterization, the laser beam was split into two paths: a frequency-doubled $(\lambda=400 \mathrm{~nm})$ excitation beam used for inducing the photoresponse signal in the bridge, and an $800-\mathrm{nm}$ sampling beam for monitoring the electric field penetrating an $\mathrm{EO}\left(\mathrm{LiTaO}_{3}\right)$ crystal during the electrical pulse propagation (see Fig. 77.39). The sampling beam was time delayed with respect to the excitation beam by a computer-controlled translation stage, directed between the coplanar lines through the $\mathrm{LiTaO}_{3}$ crystal less than $100 \mu \mathrm{m}$ away from the bridge, and reflected to the analyzer by a highreflectivity dielectric coating at the bottom of the $\mathrm{LiTaO}_{3}$ crystal. The sampling beam sensed the instantaneous birefringence introduced in $\mathrm{LiTaO}_{3}$ by the photogenerated transient that propagated in the CPS underneath the crystal. By varying the relative delay between the moment of photoresponse generation (excitation beam) and the signal probing (sampling beam), the whole time-domain waveform could be resolved. From the operational point of view, our EO system can be regarded as a sampling oscilloscope ${ }^{4}$ featuring $<200$-fs time resolution and $\langle 150-\mu \mathrm{V}$ voltage sensitivity, which are well below the characteristics of transients reported here.

The samples were mounted in a continuous-flow helium optical cryostat. All the experiments were carried out in a temperature range from $20 \mathrm{~K}$ to $80 \mathrm{~K}$ with the temperature control of $\pm 0.2 \mathrm{~K}$. Our test structures were connected to dc current and voltage sources, for biasing and characterizing the in-situ current voltage $(I-V)$ of the junction and the microbridge, as well as to a 14-GHz-bandwidth oscilloscope for aligning the experiment and monitoring the bolometric response.

\section{Experimental Results}

The studied YBCO thin films, junctions, and microbridges exhibited standard, high-quality characteristics. The superconducting transition temperature $T_{c}$ of as-prepared, 100-nmthick films was in the $85 \mathrm{~K}$ to $87 \mathrm{~K}$ range. The $I-V$ characteristics of a bicrystal Josephson junction on a $\mathrm{MgO}$ substrate showed an RSJ-like behavior with $I_{C}=700 \mu \mathrm{A}$ and $I_{c} R_{N}=2.5 \mathrm{mV}$, and $I_{c}=40 \mu \mathrm{A}$ and $I_{c} R_{N}=0.1 \mathrm{mV}$, at $4.2 \mathrm{~K}$ and $77 \mathrm{~K}$, respectively (Fig. 77.40). The microbridge $I-V$ curves exhibited a flux-flow transition into a resistive state at $J_{c} \approx 10^{6} \mathrm{~A} / \mathrm{cm}^{2}$ at $77 \mathrm{~K}$. At higher bias currents, hot-spot formation occurred and the microbridge was driven into a switched (resistive) state. The microbridges were biased with a voltage source to prevent their destruction. 4
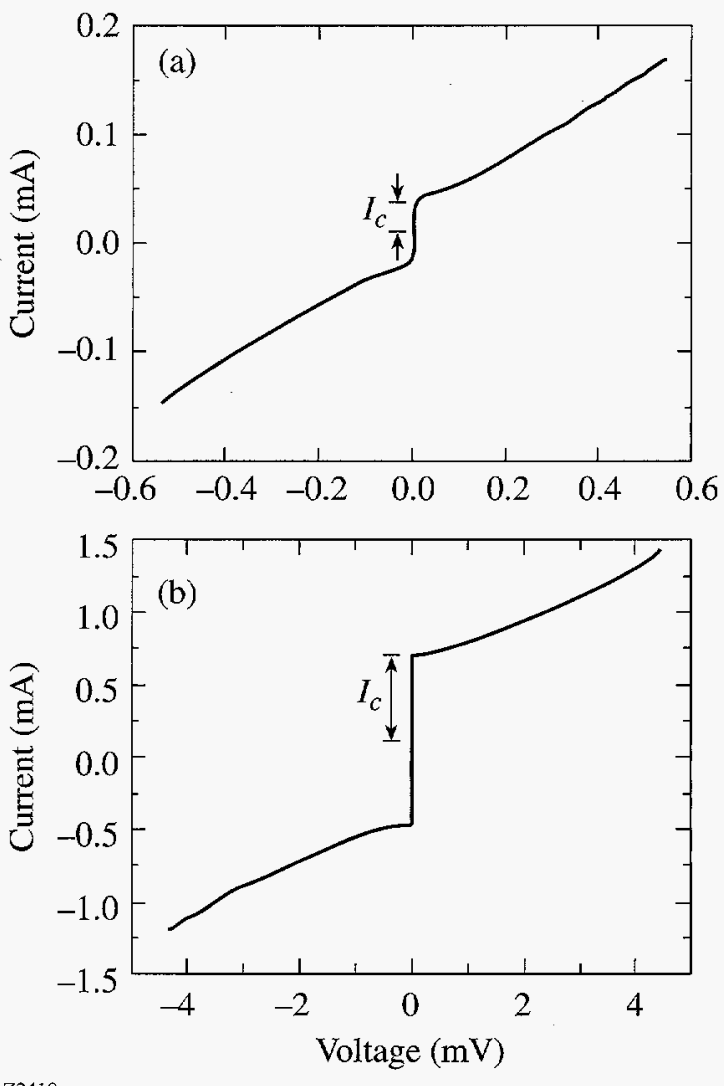

Figure 77.40

Current-voltage characteristics of a bicrystal Josephson junction with $I_{C}$ $=40 \mu \mathrm{A}$ and $I_{c}=700 \mu \mathrm{A}$ at $77 \mathrm{~K}$ and $4.2 \mathrm{~K}$, respectively. 
Figure 77.41 shows the EO sampling measurement of a 2.1-ps-wide electrical transient, typical for the microbridge biased in the resistive state. ${ }^{5}$ Pulses of this type were used in all experiments presented in this article. Typically, the pulse amplitude was about $25 \mathrm{mV}$, which, for the approximately $80-\Omega$ CPS line, corresponded to an $\sim 300-\mu$ A current pulse.

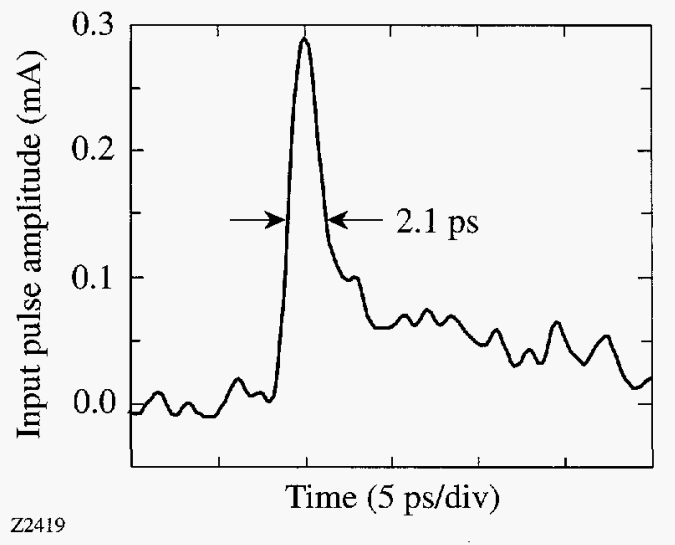

Figure 77.41

Photogenerated electrical transient of the YBCO microbridge.

The pulse from Fig. 77.41 was applied to the junction, and the resulting signal was electro-optically detected approximately $50 \mu \mathrm{m}$ after the junction (see Fig 77.39). Experiments were performed at $20 \mathrm{~K}$, and the junction $I_{c}$ was $650 \mu \mathrm{A}\left(I_{c} R_{N}\right.$ $\approx 2 \mathrm{mV}$ ). To elicit a junction response from the measured output, we used the following procedure: We biased our junction in five different points on the $I-V$ curve, namely at $0 I_{c}$, $\pm 0.7 I_{c}$, and $\pm 1.5 I_{c}$, and recorded the response. The " + " bias corresponded to the positive amplitude of the input pulse, while in the "-" polarity, the junction was biased in the opposite direction to the excitation pulse. Figure 77.42(a) shows the normalized transient responses from the junction at $0 I_{c},+0.7 I_{c}$, and $+1.5 I_{c}$. We note that the initial, positive part of each response overlaps, while some differences are visible in the remainder of the pulse. After the initial oscillatory transient, we observe additional oscillations, which are very noisy and, therefore, difficult to analyze. A similar set of responses was collected for the $-0.7 I_{C},-1.5 I_{c}$, and $0 I_{c}$ bias points and is shown in Fig. 77.42(b). All signals presented in Fig. 77.42 look very similar since they are dominated by the inductive response of the junction leads combined with the CPS resistance. Thus, the oscillatory transient with no bias applied to the junction (thin lines in Fig. 77.42) will be referred to as the feedthrough in further discussion.

\section{Discussion}

We believe that despite the fact that due to the design constraints all the waveforms presented in Fig. 77.42 look very similar and are dominated by the feedthrough signal, they contain information about switching dynamics of the YBCO Josephson junction; thus, we have subtracted the $0 I_{c}$ feedthrough signal from each measured waveform (results are shown in Fig. 77.43). For clarity, we separately showed the responses for the $\pm 1.5 I_{c}$ biased junction [Fig. 77.43(a)] and for $\pm 0.7 I_{c}$ [Fig. 77.43(b)]. In both cases, we overlaid the traces with the $0 I_{C}$ signal, which can be regarded as the zero-time reference for the junction response. From Fig. 77.43(a) we observe that when the junction is in the voltage state, the junction response for both bias polarities is positive and consists of an $\sim 1$-ps-wide transient with a turn-on delay of $0.7 \mathrm{ps}$. The response is consistent with our simulations (not shown)
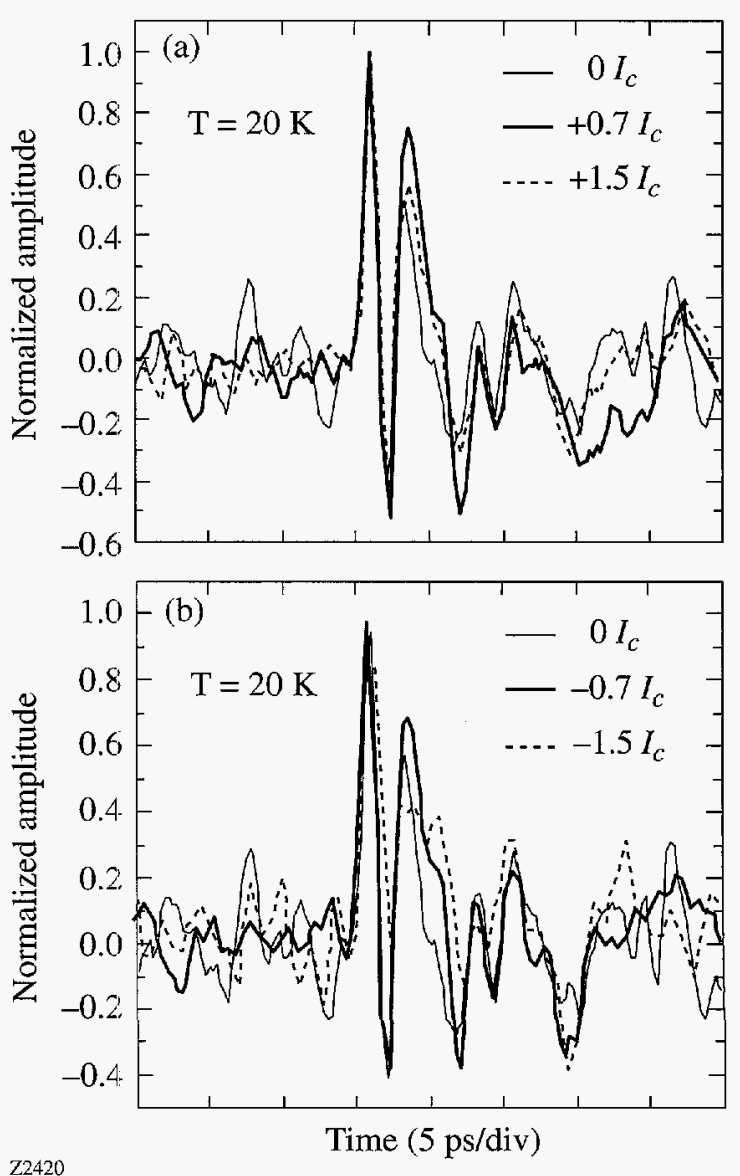

Figure 77.42

The test structure response to the ultrafast input pulse measured for different junction biases on the CPS line $50 \mu \mathrm{m}$ after the Josephson junction. All signals are normalized to the zero-bias feedthrough signal. 

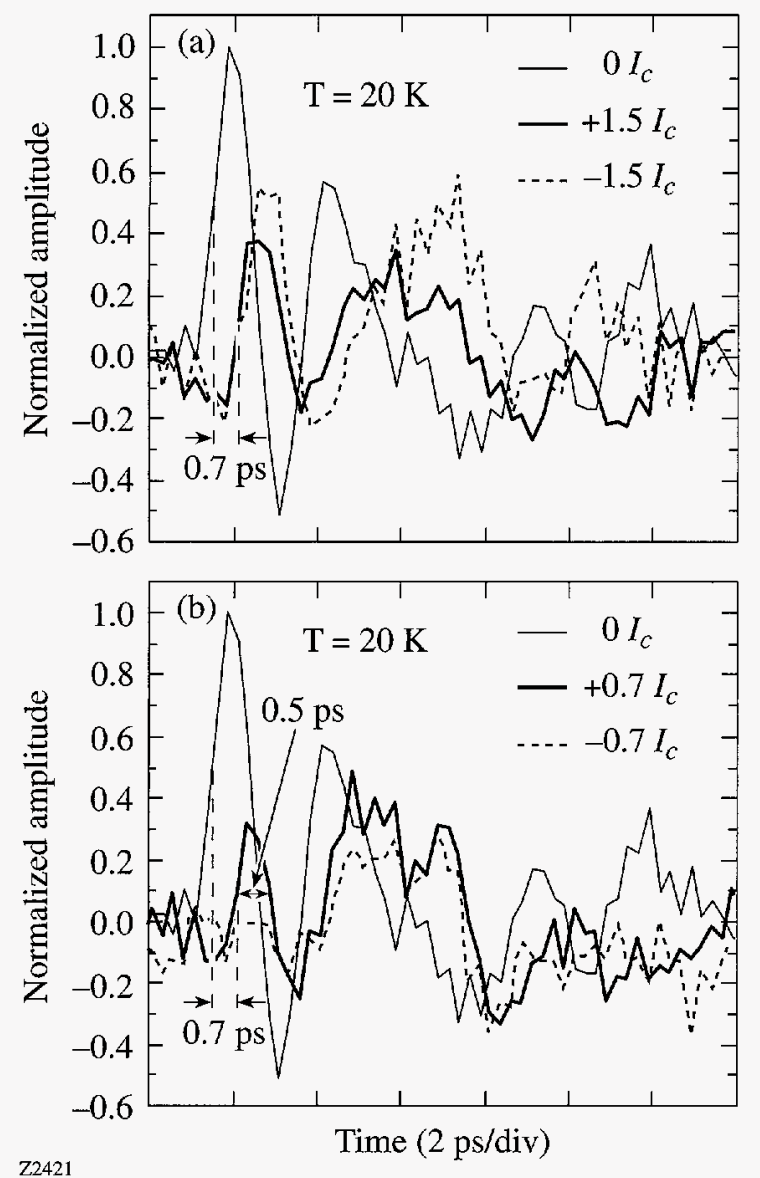

Figure 77.43

Response of the Josephson junction to the 2.1-ps electrical input pulse measured for different junction biases.

that in the voltage state, when junction current is oscillating with the frequency corresponding to the bias voltage, a small (compared to the bias) current-pulse perturbation generates a signal always in the direction of the excitation pulse polarization. In addition, the signal response is delayed with respect to the excitation.

The transients obtained for the junction biased in the superconducting state $\left( \pm 0.7 I_{c}\right)$ are shown in Fig. $77.43(\mathrm{~b})$. When the $\mathrm{dc}$ bias polarity is in the same direction $\left(+0.7 I_{c}\right)$ as the $0.46 I_{C}$ input-pulse amplitude, the excitation "knocks" the junction out of the zero-voltage state and we observe an SFQ-like, 0.5-pswide transient. We note that, at $20 \mathrm{~K}, I_{c} R_{N} \approx 2 \mathrm{mV}$; thus, the SFQ amplitude is expected to be $\sim 4 \mathrm{mV}$, which leads to $\sim 0.5$-ps SFQ width, in good agreement with our observation. In the case of the negative bias $\left(-0.7 I_{c}\right)$, however, the incoming pulse is unable to switch the junction and no response is detected. The measured $\tau_{D}$ of the $+0.7 I_{C}$ biased junction is $0.7 \mathrm{ps}$, the same as for the $\pm 1.5 I_{c}$ case. We must finally note that signals observed in Figs. 77.43(a) and 77.43(b) after the initial pulse responses discussed above are associated with the secondary input-pulse reflections and resulting secondary switching of our junction. Their detailed analysis and comparison with numerical circuit simulations will be presented in a later publication.

\section{Conclusion}

Picosecond electrical pulses, optically generated in currentbiased YBCO microbridges, were used to excite the response of a bicrystal Josephson junction placed in the YBCO superconducting coplanar transmission line. The transients recorded just past the junction contained large feedthrough signals, but the junction response could be separated by subtracting the feedthrough from the signals obtained under different bias conditions. As a result, we were able to observe single-picosecond switching of HTS Josephson junctions, as well as to measure the junction turn-on delay time. Our findings provide confirmation of the potential of YBCO for ultrafast optical and electrical transient detection and processing. In the future, however, a new feedthrough-free test structure representing YBCO Josephson-junction coplanar transmission line is needed. The development of such a circuit is currently underway.

\section{ACKNOWLEDGMENT}

This research is supported by the Office of Naval Research grant N0001498-1-0080 and also from the Frank Horton Graduate Fellowship Program.

\section{REFERENCES}

1. V. F. Elesin and Yu. V. Kopaev, Sov. Phys. Usp. 24, 116 (1981), and references therein.

2. R. Sobolewski, D. P. Butler, T. Y. Hsiang, C. V. Stancampiano, and G. A. Mourou, Phys. Rev. B 33, 4604 (1986), and references therein.

3. F. A. Hegmann, D. Jacobs-Perkins, C.-C. Wang, S. H. Moffat, R. A. Hughes, J. S. Preston, M. Currie, P. M. Fauchet, T. Y. Hsiang, and R. Sobolewski, Appl. Phys. Lett. 67, 285 (1995).

4. M. Lindgren, M. Currie, C. A. Williams, T. Y. Hsiang, P. M. Fauchet, R. Sobolewski, S. H. Moffat, R. A. Hughes, J. S. Preston, and F. A. Hegmann, IEEE J. Sel. Top. Quantum Electron. 2, 668 (1996).

5. M. Lindgren, M. Currie, C. Williams, T. Y. Hsiang, P. M. Fauchet, R. Sobolewski, S. H. Moffat, R. A. Hughes, J. S. Preston, and F. A. Hegmann, IEEE Trans. Appl. Supercond. 7, 3422 (1997).

6. M. Lindgren, M. Currie, C. A. Williams, T. Y. Hsiang, P. M. Fauchet, R. Sobolewski, S. H. Moffat, R. A. Hughes, J. S. Preston, and F. A. Hegmann, "Intrinsic Picosecond Response Times of $\mathrm{Y}-\mathrm{Ba}-\mathrm{Cu}-\mathrm{O}$ Superconducting Photodetectors," to be published in Applied Physics Letters. 
7. R. Sobolewski, in Superconducting and Related Oxides: Physics and Nanoengineering III, edited by I. Bozovic and D. Pavuna (SPIE, Bellingham, WA, 1998), Vol. 3481, pp. 480-491.

8. S. H. Dhong and T. Van Duzer, IEEE Trans. Electron Devices ED-27, 1965 (1980).

9. M. Darula and M. Kedro, J. Low Temp. Phys. 78, 287 (1990).
10. D. G. McDonald et al., IEEE Trans. Electron Devices ED-27, 1945 (1980).

11. K. K. Likharev and V. K. Semenov, IEEE Trans. Appl. Supercond. 1, 3 (1991).

12. R. A. Hegmann, R. A. Hughes, and J. S. Preston, Appl. Phys. Lett. 64, 3172 (1994). 


\section{Publications and Conference Presentations}

\section{Publications}

R. Betti, V. Lobatchev, and R. L. McCrory, "Feedout, Secular Distortion, and R-T Seeding Induced by Long-Wavelength Perturbations in Accelerated Planar Foils," Phys. Rev. Lett. 81, 5560 (1998).

S.-H. Chen, B. M. Conger, J. C. Mastrangelo, and A. S. Kende, "Synthesis and Optical Properties of Thermotropic Polythiophene and Poly(p-Phenylene) Derivatives," Macromolecules 31, 8051 (1998).

T. J. B. Collins, H. L. Helfer, and H. M. Van Horn, "A Model for Quasi-Periodic Oscillations in Cataclysmic Variables Based on Boundary-Layer Oscillations," Astrophys. J. 508, L159 (1998).

B. M. Conger, J. C. Mastrangelo, D. Katsis, and S.-H. Chen, "Fluorescence of Pyrenyl and Carbazolyl Derivatives in Liquid Solution and Solid Film," J. Phys. Chem. A 102, 9213 (1998).

S. Cremer, C. P. Verdon, and R. D. Petrasso, "Tertiary Proton Diagnostics in Future ICF Experiments," Phys. Plasmas 5, 4009 (1998).

M. Currie, R. Sobolewski, and T. Y. Hsiang, "Subterahertz Signal Crosstalk in Transmission Line Interconnects," Appl. Phys. Lett. 73, 1910 (1998).

F. Dahmani, A. W. Schmid, J. C. Lambropoulos, and S. J. Burns, "Dependence of Birefringence and Residual Stress Near Laser-Induced Cracks in Fused Silica on Laser Fluence and on Laser-Pulse Number," Appl. Opt. 37, 7772 (1998).
K. S. Il'in, I. I. Milostnaya, A. A. Verevkin, G. N. Gol'tsman, E. M. Gershenzon, and R. Sobolewski, "Ultimate Quantum Efficiency of a Superconducting Hot-Electron Photodetector," Appl. Phys. Lett. 73, 3938 (1998).

A.V. Kanaev and C. J. McKinstrie, "Exact Green's Function for a Class of Parametric Instabilities," Phys. Plasmas 5, 4511 (1998).

R. W. Short and A. Simon, "Collisionless Damping of Localized Plasma Waves in Laser-Produced Plasmas and Application to Stimulated Raman Scattering in Filaments," Phys. Plasmas 5, 4134 (1998).

R. W. Short and A. Simon, "Landau Damping and TransitTime Damping of Localized Plasma Waves in General Geometries," Phys. Plasmas 5, 4124 (1998).

V. A. Smalyuk, T. R. Boehly, D. K. Bradley, V. N. Goncharov, J. A. Delettrez, J. P. Knauer, D. D. Meyerhofer, D. Oron, and D. Shvarts, "Saturation of the Rayleigh-Taylor Growth of Broad-Bandwidth, Laser-Imposed Nonuniformities in Planar Targets," Phys. Rev. Lett. 81, 5342 (1998).

B. Yaakobi, F. J. Marshall, and D. K. Bradley, "Pinhole-Array X-Ray Spectrometer for Laser-Fusion Experiments," Appl. Opt. 37, 8074 (1998). 


\section{Forthcoming Publications}

E. L. Alfonso, S.-H. Chen, R. Q. Gram, D. R. Harding, and F.-Y. Tsai, "Fabrication of Polyimide Shells by Vapor Phase Deposition for Use as ICF Targets," to be published in Fusion Technology.

A. Babushkin, J. H. Kelly, C. T. Cotton, M. Labuzeta, M. Miller, T. A. Safford, R. G. Roides, W. Seka, I. Will, M. D. Tracy, and D. L. Brown, "Compact $\mathrm{Nd}^{3+}$-Based Laser System with Gain $G_{\mathrm{ss}} 10^{13}$ and 20-J Output Energy," to be published in SPIE's Proceedings of Solid State Lasers for Application (SSLA) to Inertial Confinement Fusion, 3rd Annual International Conference, Monterey, CA, 7-12 June 1998.

T. R. Boehly, A. Babushkin, D. K. Bradley, R. S. Craxton, J.A. Delettrez, R. Epstein, T. J. Kessler, J. P. Knauer, R.L. McCrory, P. W. McKenty, D. D. Meyerhofer, S. P. Regan, W. Seka, S. Skupsky, V. A. Smalyuk, R. P. J. Town, and B. Yaakobi, "Laser-Uniformity and Hydrodynamic-Stability Experiments at the OMEGA Laser Facility," to be published in Laser and Particle Beams.

T. R. Boehly, R. L. McCrory, C. P. Verdon, W. Seka, S. J. Loucks, A. Babushkin, R. E. Bahr, R. Boni, D. K. Bradley, R. S. Craxton, J. A. Delettrez, W. R. Donaldson, R. Epstein, D. Harding, P. A. Jaanimagi, S. D. Jacobs, K. Kearney, R. L. Keck, J. H. Kelly, T. J. Kessler, R. L. Kremens, J. P. Knauer, D. J. Lonobile, L. D. Lund, F. J. Marshall, P. W. McKenty, D. D. Meyerhofer, S. F. B. Morse, A. Okishev, S. Papernov, G. Pien, T. Safford, J. D. Schnittman, R. Short, M. J. Shoup III, M. Skeldon, S. Skupsky, A. W. Schmid, V. A. Smalyuk, D. J. Smith, J. M. Soures, M. Wittman, and B. Yaakobi, "Inertial Confinement Fusion Experiments with OMEGA-a 30-kJ, 60-Beam UV Laser," to be published in the Proceedings of the 1997 IAEA Conference, Osaka, Japan, 10-14 March 1997.

T. R. Boehly, V. A. Smalyuk, D. D. Meyerhofer, J. P. Knauer, D. K. Bradley, R. S. Craxton, M. J. Guardalben, S. Skupsky, and T. J. Kessler, "The Reduction of Laser Imprinting Using Polarization Smoothing on a Solid-State Fusion Laser," to be published in the Journal of Applied Physics.

S.-H. Chen, D. Katsis, A. W. Schmid, J. C. Mastrangelo, T. Tsutsui, and T. N. Blanton, "Circularly Polarized Light Generated by Photoexcitation of Luminophores in Vitrified Liquid-Crystal Films," to be published in Nature.
S.-H. Chen, J. C. Mastrangelo, B. M. Conger, and D. Katsis, "Design, Synthesis, and Potential Application of Glass-Forming Functional Organic Materials," to be published in the Proceedings of the 6th International Polymer Conference, Kusatsu, Japan, 20-24 October 1997 (invited).

T. J. B. Collins, A. Frank, J. E. Bjorkman, and M. Livio, "Supernova 1987A: Rotation and a Binary Companion," to be published in Astrophysical Journal.

F. Dahmani, S. J. Burns, and J. C. Lambropoulous, "Arresting UV-Laser Damage in Fused Silica," to be published in Optics Letters.

F. Dahmani, J. C. Lambropoulos, S. Burns, S. Papernov, and A. W. Schmid, "How Small Stresses Affect 351-nm Damage Onset in Fused Silica," to be published in SPIE's Proceedings of the XXX Annual Symposium on Optical Materials for High Power Lasers, Boulder, CO, 28 September-1 October 1998.

F. Dahmani, J. C. Lambropoulos, A. W. Schmid, S. J. Burns, and C. Pratt, "Nanoindentation Technique for Measuring Residual Stress Field Around a Laser-Induced Crack in Fused Silica," to be published in the Journal of Materials Science.

F. Dahmani, J. C. Lambropoulos, A. W. Schmid, S. Papernov, and S. J. Burns, "Fracture of Fused Silica with 351-nm-LaserGenerated Surface Cracks," to be published in the Journal of Materials Research.

F. Dahmani, A. W. Schmid, J. C. Lambropoulos, and S. J. Burns, "Lifetime Prediction of Laser-Precracked Fused Silica Subjected to Subsequent Cyclic Laser Pulses," to be published in the Journal of Materials Science.

O. M. Efimov, L. B. Glebov, S. Papernov, A. W. Schmid, and E. Van Stryland "Laser-Induced Damage of Photo-ThermoRefractive Glasses for Optical Holographic Elements Writing," to be published in SPIE's Proceedings of the XXX Annual Symposium on Optical Materials for High Power Lasers, Boulder, CO, 28 September-1 October 1998.

V. N. Goncharov, "Ablation Front Oscillations During the Shock Transit Time," to be published in Physical Review Letters. 
K. Green, W. Seka, M. D. Skeldon, R. L. Keck, A. V. Okishev, and R. Sobolewski, "Transient Bandwidth Analysis of Photoconductive Microwave Switches Implemented in the OMEGA Pulse-Shaping System," to be published in SPIE's Proceedings of Solid State Lasers for Application (SSLA) to Inertial Confinement Fusion, 3rd Annual International Conference, Monterey, CA, 7-12 June 1998.

D. Katsis, A. W. Schmid, and S.-H. Chen, "Mechanistic Insight into Circularly Polarized Photoluminescence from a ChiralNematic Film," to be published in Liquid Crystals.

J. P. Knauer, R. Betti, D. K. Bradley, T. R. Boehly, T. J. B. Collins, V. N. Goncharov, P. W. McKenty, D. D. Meyerhofer, V.A. Smalyuk, C.P. Verdon, S. G. Glendinning, D. H. Kalantar, and R. G. Watt, "Single-Mode Rayleigh-Taylor Growth-Rate Measurements with the OMEGA Laser System," to be published in Physics of Plasmas.

E. M. Korenic, S. D. Jacobs, S. M. Faris, and L. Li, "Cholesteric Liquid Crystal Flakes-A New Form of Domain," to be published in Molecular Crystals and Liquid Crystals.

E. M. Korenic, S. D. Jacobs, S. M. Faris, and L. Li, "Cholesteric Liquid Crystal Transmission Profile Asymmetry," to be published in Molecular Crystals and Liquid Crystals.

J. M. Larkin, W. R. Donaldson, T. H. Foster, and R. S. Knox, "Reverse Intersystem Crossing from a Triplet State of Rose Bengal Populated by Sequential 532- + 1064-nm Laser Excitation," to be published in Chemical Physics.

M. Lindgren, M. Currie, C. Williams, T. Y. Hsiang, P. M. Fauchet, R. Sobolewski, S. H. Moffat, R. A. Hughes, J. S. Preston, and F. A. Hegmann, "Intrinsic Picosecond Response Times of Y-Ba-Cu-O Superconducting Photodetectors," to be published in Applied Physics Letters.

M. Lindgren, W.-S. Zeng, M. Currie, R. Sobolewski, S. Cherednichenko, B. Voronov, and G. N. Gol'tsman, "Picosecond Response of a Superconducting Hot-Electron NbN Photodetector," to be published in Applied Superconductivity.

J. A. Marozas, "The Cross-Phase Modulation Between Two Intense Orthogonally Polarized Laser Beams Co-Propagating through a Kerr-like Medium," to be published in SPIE's Proceedings of Solid State Lasers for Application (SSLA) to Inertial Confinement Fusion, 3rd Annual International Conference, Monterey, CA, 7-12 June 1998.
F. J. Marshall and G. R. Bennett, "A High-Energy X-Ray Microscope for Inertial Confinement Fusion," to be published in the Review of Scientific Instruments.

K. L. Marshall, J. Haddock, N. Bickel, D. Singel, and S. D. Jacobs, "Angular-Scattering Characteristics of Ferroelectric Liquid Crystal Electro-Optical Devices Operating in the TSM and ESM Modes," to be published in Applied Optics.

R. L. McCrory and J. M. Soures, "Status of Direct-Drive Inertial Confinement Fusion Research at the Laboratory for Laser Energetics," to be published in the Proceedings of the 2nd Symposium on Current Trends in International Fusion Research Review and Assessment, Washington, DC, 10-14 March 1997 (invited).

P. W. McKenty, "Direct-Drive Capsule Requirements for the National Ignition Facility and OMEGA Laser Systems," to be published in Fusion Technology.

P. W. McKenty and M. D. Wittman, "Characterization of Thick Cryogenic Layers Using an Interferometric Imaging System," to be published in Fusion Technology.

C. J. McKinstrie, R. E. Giacone, and E. A. Startsev, "Accurate Formulas for the Landau Damping Rates of Electrostatic Waves," to be published in Physics of Plasmas.

S. J. McNaught and D. D. Meyerhofer, "Precision Measurement of Electron Initial Conditions for Tunneling Ionization in an Elliptically Polarized Laser," to be published in the Proceedings of the Sixteenth International Conference on Atomic Physics, Windsor, Ontario, Canada, 3-7 August 1998.

B. Nodland and C. J. McKinstrie, "Propagation of a Short Laser Pulse in a Plasma," to be published in Physical Review E.

A. V. Okishev, M. D. Skeldon, and W. Seka, "Multipurpose, Diode-Pumped Nd:YLF Laser for OMEGA Pulse Shaping and Diagnostics Applications," to be published in SPIE's Proceedings on Solid State Lasers for Application (SSLA) to Inertial Confinement Fusion, 3rd Annual International Conference, Monterey, CA, 7-12 June 1998.

S. P. Regan, D. K. Bradley, J. J. Carroll III, A. V. Chirokikh, R. S. Craxton, R. P. Drake, D. D. Meyerhofer, W. Seka, R. W. Short, A. Simon, R. P. J. Town, and B. Yaakobi, "Laser-Plasma Interactions in Long-Scale-Length Plasmas Under Direct- 
Drive National Ignition Facility Conditions," to be published in Physics of Plasmas.

A. L. Rigatti, D. J. Smith, A. W. Schmid, S. Papernov, and J. H. Kelly, "Damage in Fused-Silica Spatial-Filter Lenses on the OMEGA Laser System," in SPIE's Proceedings of the XXX Annual Symposium on Optical Materials for High Power Lasers, Boulder, CO, 28 September-1 October 1998.

A. B. Shorey, L. L. Gregg, H. J. Romanofsky, S. R. Arrasmith, I. A. Kozhinova, and S. D. Jacobs, "A Study of Material Removal During Magnetorheological Finishing (MRF), " to be published in Optical Manufacturing and Testing.

M. D. Skeldon, A. V. Okishev, R. L. Keck, W. Seka, and S. A. Letzring, "An Optical Pulse Shaping System Based on Aperture-Coupled Stripline for OMEGA Pulse Shaping Applications," to be published in SPIE's Proceedings of Solid State Lasers for Application (SSLA) to Inertial Confinement Fusion, 3rd Annual International Conference, Monterey, CA, 7-12 June 1998.

M. D. Skeldon, R. Saager, and W. Seka, "Quantitative PumpInduced Wavefront Distortions in Laser-Diode- and FlashLamp-Pumped Nd:YLF Laser Rods," to be published in the IEEE Journal of Quantum Electronics.

S. Skupsky and R. S. Craxton, "Irradiation Uniformity for High-Compression Laser Fusion Experiments," to be published in Physics of Plasmas.

V. A. Smalyuk, T. R. Boehly, D. K. Bradley, V. N. Goncharov, J. A. Delettrez, J. P. Knauer, D. D. Meyerhofer, D. Oron, D. Shvarts, and Y. Srebro, "Nonlinear Evolution of BroadBandwidth, Laser-Imposed Nonuniformities in Planar Targets," to be published in Physics of Plasmas.

V. A. Smalyuk, T. R. Boehly, D. K. Bradley, J. P. Knauer, and D. D. Meyerhofer, "Characterization of an X-Ray Radiographic System Used for Laser-Driven Planar Target Experiments," to be published in Review of Scientific Instruments.
D. J. Smith, J. A. Warner, N. E. LeBarron, T. J. Kessler, S. LaDelia, J. P. Knauer, D. D. Meyerhofer, D. Oron, and D. Shvarts, "The Development of Ion-Etched Phase Plates," to be published in Applied Optics.

D. J. Smith, J. A. Warner, N. E. LeBarron, and S. LaDelia, "Production of Distributed Phase Plates Using an Energetic Ion Process," to be published in SPIE's Proceedings of the XXX Annual Symposium on Optical Materials for High Power Lasers, Boulder, CO, 28 September-1 October 1998.

R. Sobolewski, "Ultrafast Dynamics of Nonequilibrium Quasiparticles in High-Temperature Superconductors," to be published in Superconducting and Related Oxides: Physics and Nanoengineering III.

B. Yaakobi and F. J. Marshall, "Imaging the Cold, Compressed Shell in Laser Implosions Using the $\mathrm{K} \alpha$ Fluorescence of a Titanium Dopant," to be published in the Journal of Quantitative Spectroscopy and Radiative Transfer.

B. Yaakobi, F. J. Marshall, and D. K. Bradley, "K $\alpha$ Cold Target Imaging and Preheat Measurement Using PinholeArray X-Ray Spectrometer," to be published in the Review of Scientific Instruments.

L. Zheng and D. D. Meyerhofer, "Self- and Cross-Plate Modulation Coefficients in a KDP Crystal Measured by a Z-Scan Technique," to be published in the Journal of the Optical Society of America B.

J. D. Zuegel and W. Seka, "Upconversion and Reduced ${ }^{4} F_{3 / 2}$ Upper-State Lifetime in Intensely Pumped Nd:YLF," to be published in Applied Optics: Lasers, Photonics \& Environmental Optics. 
J. L. Chaloupka and D. D. Meyerhofer, "A Single-Beam, HighField Trap for Energetic Electrons," 1998 OSA Annual Meeting/ILS-XIV, Baltimore, MD, 4-9 October 1998.

J. M. Soures, R. L. McCrory, P. B. Radha, R. Betti, W. Bittle, T. R. Boehly, R. Boni, D. K. Bradley, T. J. B. Collins, R. S. Craxton, J. A. Delettrez, W. R. Donaldson, R. Epstein, V. Yu. Glebov, V. N. Goncharov, D. R. Harding, P. A. Jaanimagi, R. L. Keck, J. H. Kelly, T. J. Kessler, J. P. Knauer, C. K. Li, S. J. Loucks, F. J. Marshall, P. W. McKenty, D. D. Meyerhofer, S. F. B. Morse, S. Padalino, R. Petrasso, S. Regan, W. Seka, R. W. Short, A. Simon, S. Skupsky, D. J. Smith, R. P. J. Town, B. Yaakobi, and J. D. Zuegel, "Recent Advances in DirectDrive ICF Target Physics at the Laboratory for Laser Energetics," 1998 IAEA Conference, Yokohama, Japan, 19-24 October 1998.

The following presentations were made at the 40th Annual Meeting APS Division of Plasma Physics, New Orleans, LA, 16-20 November 1998:

P. Amendt, R. E. Turner, O. Landen, S. G. Glendinning, D. Kalantar, M. Cable, J. Colvin, C. Decker, L. Suter, R. Wallace, D. K. Bradley, S. F. B. Morse, G. Pien, W. Seka, and J. M. Soures, "High Convergence Indirect Drive Implosions on OMEGA: Design and Simulations."

C. A. Back, J. D. Bauer, R. E. Turner, B. F. Lasinski, L. J. Suter, O. L. Landen, W. W. Hsing, and J. M. Soures, "Temporally and Radially Resolved Breakout of Heat Wave in Radiatively Heated Foam Targets."

C. W. Barnes, D. L. Tubbs, J. B. Beck, J. A. Oertel, N. Shambo, S. A. Voss, R. G. Watt, T. R. Boehly, D. K. Bradley, and J. P. Knauer, "Direct-Drive Cylindrical Implosions on the OMEGA Laser."

G. R. Bennett, J. M. Wallace, T. J. Murphy, A. A. Hauer, J. A. Oertel, D. C. Wilson, P. L. Gobby, N. D. Delamater, R. E. Chrien, R. S. Craxton, and J. D. Schnittman, "High Convergence Implosions Within Tetrahedral Hohlraums."
R. Betti and E. Fedutenko, "Stable Regimes for External Modes in High- $\beta$ Tokamak Plasmas."

T. R. Boehly, V. A. Smalyuk, O. Gotchev, J. P. Knauer, D. D. Meyerhofer, D. K. Bradley, J. A. Delettrez, S. Skupsky, and R. P. J. Town, "The Effect of Pulse Shape and Beam Smoothing on Laser Imprinting."

D. K. Bradley, J. A. Delettrez, S. P. Regan, S. Skupsky, and D. D. Meyerhofer, "Spherical Rayleigh-Taylor Experiments on the 60-Beam OMEGA Laser System Using the Burnthrough Technique."

T. J. B. Collins, J. P. Knauer, S. Skupsky, and C. P. Verdon, "Control of Ablation Velocity Through Prepulses in DirectDrive ICF."

R. S. Craxton, S. Skupsky, A. Babushkin, J. H. Kelly, T. J. Kessler, J. M. Soures, and J. D. Zuegel, "Enhanced Beam Smoothing on OMEGA and the NIF."

J. A. Delettrez, V. N. Goncharov, S. Skupsky, T. R. Boehly, D. K. Bradley, J. P. Knauer, D. D. Meyerhofer, S. P. Regan, and V. A. Smalyuk, "The Effect of Pulse Shape on Laser Imprint and SSD Smoothing."

J. Dirrenberger, V. Lobatchev, and R. L. McCrory, "Seeds and Early Development of the Rayleigh-Taylor Instability in Laser-Accelerated Targets."

R. Elton, E. Iglesias, H. Griem, G. Pien, D. K. Bradley, J. A. Delettrez, and R. Epstein, "Early-Time Extreme-UV Emission from OMEGA Plasmas."

R. Epstein, T. J. B. Collins, J. A. Delettrez, S. Skupsky, and R. P. J. Town, "Simulation of the Radiative Preheat of Target Foils and Shells in Laser-Driven Ablation and Implosion Experiments."

Y. Fisher, T. R. Boehly, D. K. Bradley, D. R. Harding, D. D. Meyerhofer, and M. D. Wittman, "Shinethrough Properties of Various Barrier-Layer Materials." 
C. G. R. Geddes, J. Sanchez, G. Collins, and P. W. McKenty, "Interferometric Characterization of Hydrogen Ice Layers in NIF-Scale Targets."

R. E. Giacone, C. J. McKinstrie, and E. A. Startsev, "Accurate Formulas for the Landau Damping Rates of Electrostatic Waves."

V. Yu. Glebov, J. P. Knauer, F. J. Marshall, P. W. McKenty, D. D. Meyerhofer, N. S. Rogers, C. Stoeckl, M. D. Cable, and R. E. Turner, "Recent $\rho R$ Measurements on OMEGA Using the MEDUSA Scintillator Array."

V. N. Goncharov, R. Betti, R. L. McCrory, and C. Cherfils, "Linear Stability Analysis of Ablation Fronts During the Shock Transit Time."

D. Haynes, C. Hooper, N. Delamater, C. Barnes, J. Oertel, G. Pollak, D. Tubbs, R. Watt, T. R. Boehly, D. K. Bradley, P. A. Jaanimagi, and J. P. Knauer, "X-Ray Spectroscopy of Directly Driven Cylindrical Implosions."

D. G. Hicks, C. K. Li, F. H. Sequin, R. D. Petrasso, J. M. Soures, D. R. Harding, D. D. Meyerhofer, W. Seka, A. Simon, R. W. Short, T. W. Phillips, T. C. Sangster, M. D. Cable, T. P. Bernat, and J. D. Schnittman, "Measurement of Accelerated Ions from OMEGA Targets."

G. C. Junkel, M. A. Gunderson, D. A. Haynes, Jr., C. F. Hooper, Jr., D. K. Bradley, J. A. Delettrez, P. A. Jaanimagi, S. P. Regan, "Multi-electron Line Broadening in Hot, Dense Plasmas Including Detailed Line Shift Calculations."

A. V. Kanaev and C. J. McKinstrie, "Exact Green Function for a Class of Parametric Instabilities."

R. L. Keck, W. R. Donaldson, W. Seka, and R. Boni, "Beam Power Matching on the OMEGA Laser."

J. P. Knauer, R. Betti, T. R. Boehly, D. K. Bradley, T. J. B. Collins, J. A. Delettrez, P. W. McKenty, D. D. Meyerhofer, V. A. Smalyuk, and R. P. J. Town, "Growth of Rayleigh-Taylor Unstable, CH Ablation Interfaces Doped with Silicon."

C. K.Li, D. G. Hicks, F. H. Sequin, R. D. Petrasso, J. M. Soures, D. R. Harding, J. P. Knauer, J. Law, P. B. Radha, S. Skupsky, S. Padilino, T. W. Phillips, T. C. Sangster, and M. D. Cable, "Measurements of Temperature and Areal Density Using Charged-Particle Spectroscopy on OMEGA."
V. Lobatchev and R. Betti, "Linear Feed-out of Rear Surface Nonuniformities in Planar Geometry."

F. J. Marshall, B. Yaakobi, D. D. Meyerhofer, R. P. J. Town, J. A. Delettrez, V. Yu. Glebov, D. K. Bradley, J. P. Knauer, M. D. Cable, and T. J. Ognibene, "Surrogate Cryogenic Target Experiments on OMEGA."

P. W. McKenty, V.Yu. Glebov, D. D. Meyerhofer, N. S. Rogers, C. Stoeckl, J. D. Zuegel, M. D. Cable, T. J. Ognibene, R. A. Lerche, and R. L. Griffith, "Neutron Burn Truncation Experiments on OMEGA."

C. J. McKinstrie and E. A. Startsev, "Analysis of the Forward and Backward Stimulated Brillouin Scattering of Crossed Laser Beams."

D. D. Meyerhofer, D. K. Bradley, J.A.Delettrez, V. Yu. Glebov, J. P. Knauer, F. J. Marshall, P. W. McKenty, S. P. Regan, S. Skupsky, C. Stoeckl, and R. P. J. Town, "Hydrodynamic Performance of Spherical CH Targets on OMEGA Using Shaped Laser Pulses."

T. J. Murphy, J. M. Wallace, K. A. Klare, N. D. Delamater, G. R. Bennett, A. A. Hauer, J. A. Oertel, S. M. Pollaine, R. S. Craxton, and J. D. Schnittman, "Analysis of Imploded Capsule Images from Spherical Hohlraums with Tetrahedral Illumination."

R. D. Petrasso, C. K. Li, D. G. Hicks, F. H. Sequin, J. M. Soures, V. Yu. Glebov, D. R. Harding, J. P. Knauer, J. Law, D. D. Meyerhofer, P. B. Radha, J. D. Schnittman, W. Seka, R. W. Short, A. Simon, S. Skupsky, C. Stoeckl, T. W. Phillips, T. C. Sangster, T.Ognibene, M. D. Cable, and S. Padilino, "ChargedParticle Spectroscopy on OMEGA: Initial Results" (invited).

P. B. Radha, S. Skupsky, J. M. Soures, and R. D. Petrasso, "A Novel Diagnostic for $\rho R$ in ICF Targets."

S. P. Regan, T. R. Boehly, D. K. Bradley, T. J. B. Collins, J. A. Delettrez, J. P. Knauer, D. D. Meyerhofer, P. W. McKenty, and V.A. Smalyuk, "A Comparison of Planar Burnthrough Experiments with Single-Mode Rayleigh-Taylor Instability Growth Rate on OMEGA."

S. P. Regan, D. K. Bradley, J. J. Carroll II, A. V. Chirokikh, R. S. Craxton, R. P. Drake, D. D. Meyerhofer, W. Seka, R. W. Short, A. Simon, R. P. J. Town, and B. Yaakobi, "Laser-Plasma Interactions in NIF Direct-Drive-Scale Plasmas" (invited). 
J. D. Schnittman, R. S. Craxton, S. M. Pollaine, R. E. Turner, J. M. Wallace, T. J. Murphy, N. D. Delamater, J. A. Oertel, A. A. Hauer, and K. A. Klare, "Capsule Implosion Symmetry in OMEGA Tetrehedral Hohlraums."

W. Seka, A. V. Chirokikh, D. D. Meyerhofer, S. P. Regan, D. K. Bradley, B. Yaakobi, R. S. Craxton, R. W. Short, and A. Simon, "Stimulated Brillouin Scattering in Direct-Drive NIF Conditions."

N.A. Shambo, W. Barnes, J.A. Oertel, R. G. Watt, T. R. Boehly, D. K. Bradley, and J. P. Knauer, "Neutron Emission from Direct-Drive Cylindrical Implosions."

R. W. Short, C. K. Li, D. G. Hicks, R. D. Petrasso, J. M. Soures, and W. Seka, "Interpretation of Ion-Acceleration Effects Observed in Charged-Particle Spectroscopy on OMEGA."

A. Simon, "Return Current Electron Beams and Their Generation of Raman Scattering."

S. Skupsky, "The Effect of Laser Nonuniformity in DirectDrive Laser-Fusion Experiments" (invited).

V.A. Smalyuk, T. R. Boehly, D. K. Bradley, J. P. Knauer, D. D. Meyerhofer, D. Oron, Y. Srebro, and D. Shvarts, "Late-Time Evolution of Broad-Bandwidth, Laser-Imposed Nonuniformities in Accelerated Foils."

J. M. Soures, D. R. Harding, P. B. Radha, S. Skupsky, C. K. Li, D. G. Hicks, R. D. Petrasso, and F. H. Sequin, "Simultaneous Measurement of Areal Density and Temperature in $\mathrm{D}^{3} \mathrm{He}$ Filled Imploding Capsules."
E. A. Startsev and C. J. McKinstrie, "Simulation of the Forward and Backward Stimulated Brillouin Scattering of Crossed Laser Beams."

C. Stoeckl, P. W. McKenty, V. Yu. Glebov, D. D. Meyerhofer, N. S. Rogers, J. D. Zuegel, M. D. Cable, T. J. Ognibene, and R. A. Lerche, "Neutron Burn History Measurements on OMEGA."

R. P. J. Town, F. J. Marshall, J. A. Delettrez, R. Epstein, P. W. McKenty, D. D. Meyerhofer, P. B. Radha, S. Skupsky, and C. Stoeckl, "OMEGA Surrogate Capsule Designs and Experiments."

R. E. Turner, P. A. Amendt, S. G. Glendinning, D. H. Kalantar, O. L. Landen, R. J. Wallace, M. D. Cable, B. A. Hammel, D. Bradley, V. Yu. Glebov, S. Morse, G. Pien, N. Rogers, W. Seka, and J. M. Soures, "X-ray Drive Symmetry and Implosion Performance in OMEGA Cylindrical Hohlraums Driven by NIF-like Multiple Cone Geometry."

J. M. Wallace, G. R. Bennett, T. J. Murphy, J. A. Oertel, P. Gobby, A. A. Hauer, W. S. Varnum, D. C. Wilson, R. S. Craxton, J. D. Schnittman, and S. M. Pollaine, "Design and Analysis of High-Convergence Capsule Implosions in OMEGA Tetrahedral Hohlraums."

B. Yaakobi, F. J. Marshall, and D. K. Bradley, "K $\alpha$ Cold Target Imaging and Preheat Measurement Using a PinholeArray X-Ray Spectrometer." 